\title{
Synthesis of bis-BN-naphthalene-fused Oxepins and Their Photoluminescence Including
}

\section{White-Light Emission}

Dawei Tian, ${ }^{\text {a }}$ Qian Li, ${ }^{a}$ Yifan Zhao, ${ }^{\text {a }}$ Zijia Wang, ${ }^{a}$ Wenbin Li, ${ }^{\text {a }}$ Shuling Xia, ${ }^{a}$ Siyang Xing, ${ }^{* a}$ Bolin Zhu, ${ }^{* a}$ Jianying Zhang, ${ }^{b}$ Chunming Cui*b

${ }^{a}$ Tianjin Key Laboratory of Structure and Performance for Functional Molecules, Key Laboratory of Inorganic-Organic Hybrid Functional Material Chemistry, Ministry of Education (Tianjin Normal University), College of Chemistry, Tianjin Normal University, Tianjin 300387, People's Republic of China.

b State Key Laboratory of Elemento-Organic Chemistry; Cooperative Innovation Center of Chemical Science and Engineering (Tianjin) Nankai University, Tianjin 300071, China

\section{Contents}

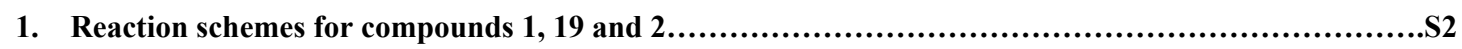

2. X-ray Crystallographic Studies of Compound 3, 8 and 16.......................................................S2

3. DFT Calculations....................................................................................................................

$\begin{array}{lllllll}\text { 4. UV-Vis } & \text { and } & \text { FL } & \text { Studies } & \text { of } & \text { Compound } & 3\end{array}$

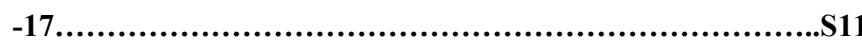

$\begin{array}{lllll}\text { 5. Temperature } & \text { Dependent } & \text { 1HNMR } & \text { Studies }\end{array}$

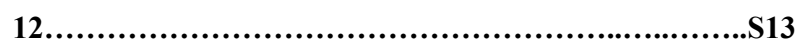

6. Scanned NMR Spectra for the New Compounds....................................................................

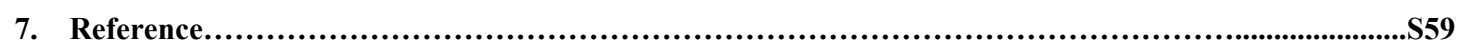


Reaction schemes for compounds 1, 19 and 2

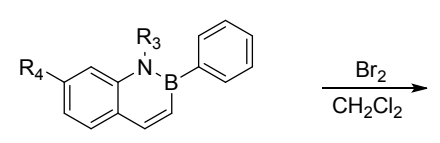

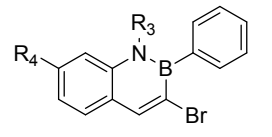

1a-f 1a $\mathrm{R}_{3}=\mathrm{H}, \mathrm{R}_{4}=\mathrm{H}$

1b $\mathrm{R}_{3}=n-\mathrm{Bu}, \mathrm{R}_{4}=\mathrm{H}$

1c $\mathrm{R}_{3}=\mathrm{C}_{6} \mathrm{H}_{5}, \mathrm{R}_{4}=\mathrm{H}$

1d $\mathrm{R}_{3}=\mathrm{Bn}, \mathrm{R}_{4}=\mathrm{H}$

1f $\mathrm{R}_{3}=\mathrm{Bn}, \mathrm{R}_{4}=\mathrm{CH}_{3}$

Scheme S1: Synthesis of brominated B-phenyl-2,1-borazaronaphthalenes

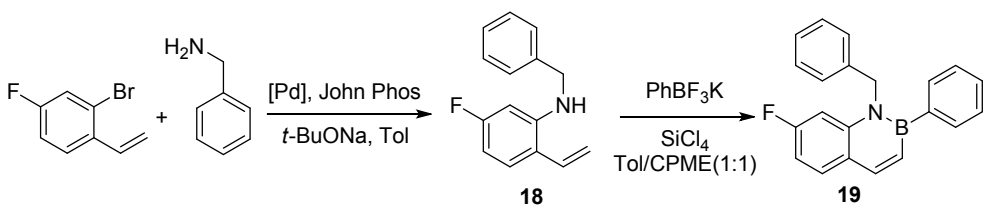

Scheme S2: Synthesis of 1-benzyl-2-phenyl-7-fluoro-2,1-Borazaronaphthalene

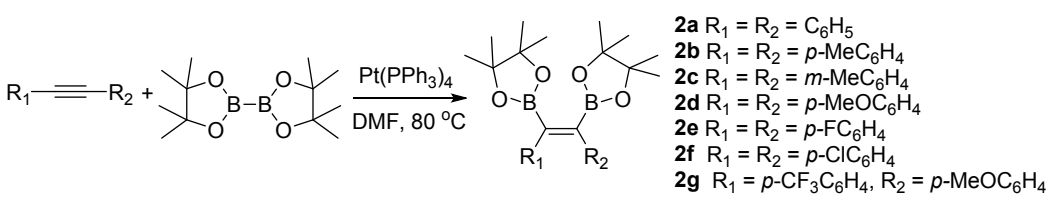

Scheme S3 : Synthesis of cis-Bis(boryl)alkenes

X-ray Crystallographic Studies of Compound 3 and 16: Single crystals of 3, 8 and 16 were achieved from the n-hexane/dichloromethane solution through slowly evaporating the solvents. Data collections for compounds $\mathbf{3}, \mathbf{8}$ and 16 were performed at $293 \mathrm{~K}, 121 \mathrm{~K}$ and $293 \mathrm{~K}$ respectively on a Rigaku Saturn CCD diffractometer using graphite-monochromated $\operatorname{MoK}(\lambda=0.71073 \AA)$ or $\operatorname{CuK}(\lambda=1.54184 \AA)$ radiation. The structures of $\mathbf{3}, 8$ and 16 were solved by use of SHELXTL program ${ }^{\mathrm{S} 1}$. Refinements were performed on $F^{2}$ anisotropically for all the non-hydrogen atoms by the full-matrixleast-squaresmethod.
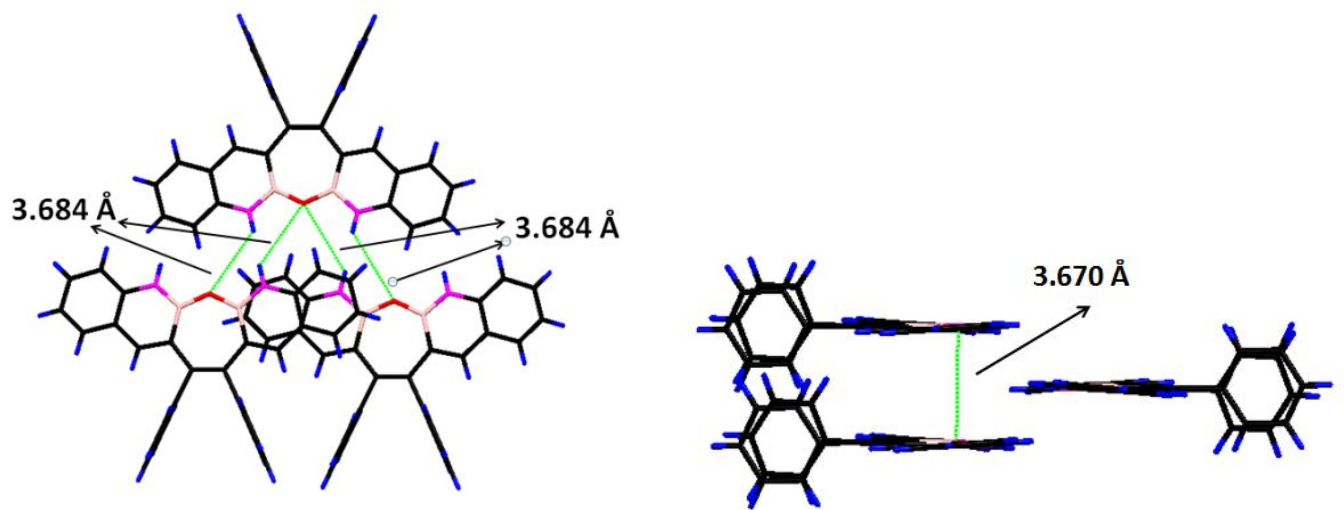

Figure S1. N-H $\cdots$ O intermolecular hydrogen bonds (left) and $\pi-\pi$ interactions (right) in crystals of 3 . 


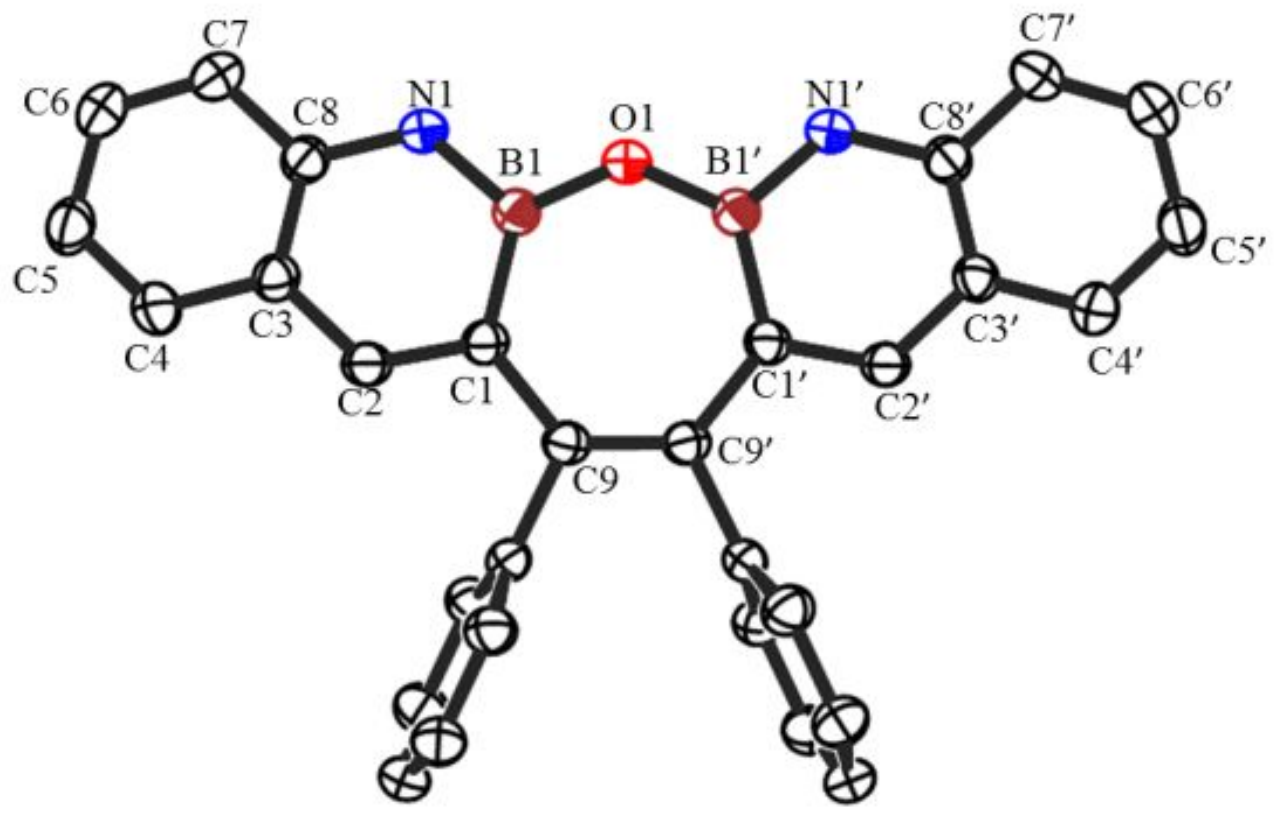

Figure S2. Molecular structure of $\mathbf{3}$ with thermal ellipsoids set at 30\% probability: top view (left) and side view (right). Hydrogen atoms have been omitted for clarity. Selected bond lengths ( $\AA$ ) and bond angles (deg): B1-N1 1.414(3), B1-C1 1.535(3), B1-O1 1.382(3), C1-C9 1.481(3), C9-C9' 1.372(3); B1-O1-B1 130.6(2), O1-B1-C1 128.2(2), B1-C1-C9 127.81(17), C1-C9-C9' 128.72(10). 


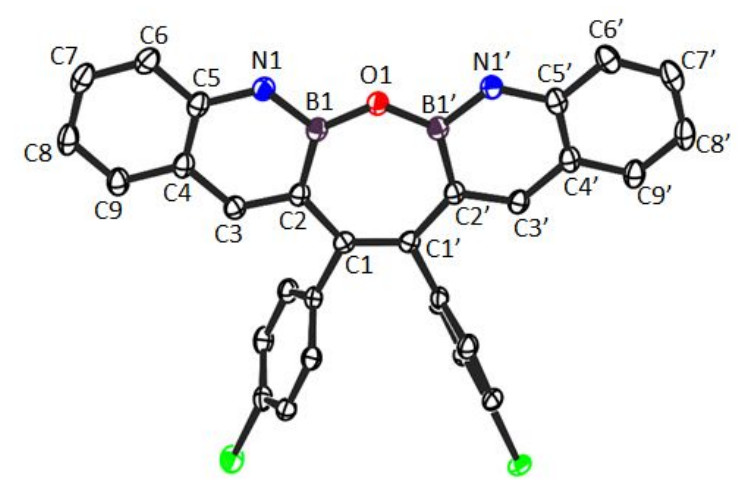

Figure S3. Molecular structure of $\mathbf{8}$ with thermal ellipsoids set at 30\% probability. Hydrogen atoms have been omitted for clarity. Selected bond lengths ( $\AA$ ) and bond angles (deg): B1-N1 1.4282(19), B1-C2 1.547(2), B1-O1 1.3737(16), C1-C2 1.4789(18), C1-C1' 1.369(3); B1-O1-B1 129.95(16), O1-B1-C2 128.91(13), B1-C2-C1 127.09(12), C2-C1-C1' 129.02(7).

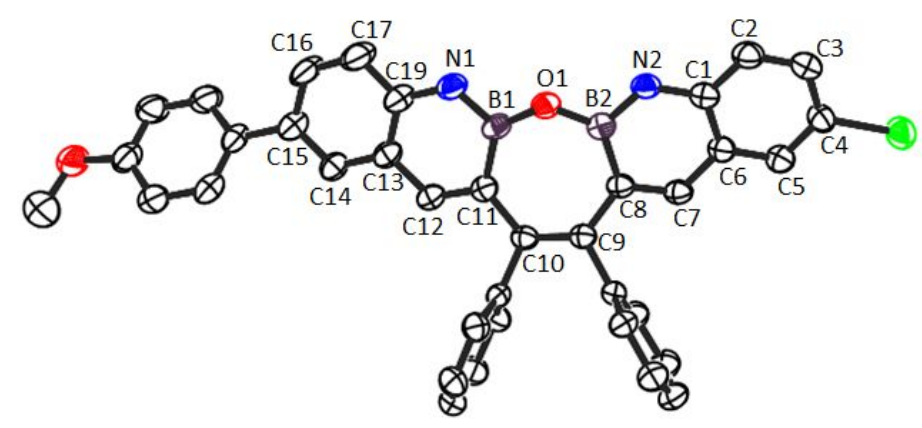

Figure S4. Molecular structure of $\mathbf{1 6}$ with thermal ellipsoids set at 30\% probability. Hydrogen atoms have been omitted for clarity. Selected bond lengths $(\AA)$ and bond angles (deg): B1-N1 1.409(7), B2-N2 1.423(7), B1-C11 1.553(7), B2-C8 1.537(7), B1-O1 1.365(7), B2-O1 1.367(7), C8-C9 1.479(6), C11-C10 1.487(7), C9-C10 1.370(7); B1-O1-B2 128.1(4), O1-B1-C11 128.5(5), B1-C11-C10 125.7(4), C11-C10-C9 127.9(4), C10-C9-C8 128.6(4), C9-C8-B2 125.7(4), C8-B2-O1 128.6(5).

Table S1. Crystallographic data and structure refinement details for 3, 8 and 16

\begin{tabular}{|l|l|l|l|}
\hline & $\mathbf{3}$ & $\mathbf{8}$ & $\mathbf{1 6}$ \\
\hline formula & $\mathrm{C}_{30} \mathrm{H}_{22} \mathrm{~B}_{2} \mathrm{~N}_{2} \mathrm{O}$ & $\mathrm{C}_{30} \mathrm{H}_{20} \mathrm{~B}_{2} \mathrm{Cl}_{2} \mathrm{~N}_{2} \mathrm{O}$ & $\mathrm{C}_{37} \mathrm{H}_{27} \mathrm{~B}_{2} \mathrm{BrN}_{2} \mathrm{O}_{2}$ \\
\hline $\mathrm{fw}$ & 448.11 & 517.00 & 633.13 \\
\hline $\mathrm{T}(\mathrm{K})$ & $293(2)$ & $120.68(12)$ & $293(2)$ \\
\hline cryst syst & monoclinic & monoclinic & monoclinic \\
\hline space group & $\mathrm{C} 2 / \mathrm{c}$ & $\mathrm{C} 2 / \mathrm{c}$ & $\mathrm{P} 2{ }_{1} / \mathrm{n}$ \\
\hline$a,(\AA)$ & $13.9056(11)$ & $13.7773(3)$ & $15.0689(7)$ \\
\hline$b,(\AA)$ & $21.1120(18)$ & $22.8718(6)$ & $8.5804(4)$ \\
\hline$c,(\AA)$ & $8.1751(7)$ & $7.77640(18)$ & $23.3930(11)$ \\
\hline
\end{tabular}




\begin{tabular}{|c|c|c|c|}
\hline$\alpha,(\operatorname{deg})$ & 90 & 90 & 90 \\
\hline$\beta,(\operatorname{deg})$ & $104.280(8)$ & $97.718(2)$ & $93.598(4)$ \\
\hline$\gamma,(\operatorname{deg})$ & 90 & 90 & 90 \\
\hline$V,\left(\AA^{3}\right)$ & $2325.9(4)$ & $2428.24(10)$ & $3018.7(2)$ \\
\hline$Z$ & 4 & 4 & 4 \\
\hline Dcalc, $\left(\mathrm{g} / \mathrm{cm}^{3}\right)$ & 1.280 & 1.414 & 1.393 \\
\hline $\mathrm{M}\left(\mathrm{mm}^{-1}\right)$ & 0.076 & 2.623 & 2.144 \\
\hline $\mathrm{F}(000)$ & 936.0 & 1064.0 & 1296.0 \\
\hline cryst size (mm) & $0.25 \times 0.22 \times 0.2$ & $0.25 \times 0.22 \times 0.2$ & $0.14 \times 0.13 \times 0.12$ \\
\hline$\lambda(\AA)$ & $\operatorname{MoK}(0.71073)$ & $\mathrm{CuK}(1.54184)$ & $\mathrm{CuK}(1.54184)$ \\
\hline $2 \theta$ range, $(\mathrm{deg})$ & 7.174 to 50.018 & 7.73 to 134.12 & 6.788 to 134.148 \\
\hline Index ranges & $\begin{array}{l}-16 \leq \mathrm{h} \leq 14 \\
-21 \leq \mathrm{k} \leq 25 \\
-8 \leq 1 \leq 9\end{array}$ & $\begin{array}{c}-16 \leq \mathrm{h} \leq 14 \\
-27 \leq \mathrm{k} \leq 26 \\
-4 \leq 1 \leq 9\end{array}$ & $\begin{array}{l}-12 \leq \mathrm{h} \leq 18 \\
-10 \leq \mathrm{k} \leq 10 \\
-27 \leq 1 \leq 27\end{array}$ \\
\hline reflns collected & 5536 & 4541 & 19640 \\
\hline indep reflns $/ R_{\text {int }}$ & $2034 / 0.0279$ & $2167 / 0.0149$ & $5393 / 0.0800$ \\
\hline params & $2034 / 0 / 160$ & $2167 / 0 / 168$ & $5393 / 6 / 398$ \\
\hline GOF on $F^{2}$ & 1.006 & 1.061 & 0.999 \\
\hline$R 1, \mathrm{w} R 2[\mathrm{I}>2 \sigma(\mathrm{I})]$ & $0.0444,0.1013$ & $0.0302,0.0777$ & $0.0698,0.1819$ \\
\hline$R 1, \mathrm{w} R 2$ (all data) & $0.0745,0.1176$ & $0.0320,0.0795$ & $0.1076,0.2161$ \\
\hline Largest diff. peak/hole / e $\AA^{-3}$ & $0.26 /-0.14$ & $0.19 /-0.21$ & $0.58 /-0.71$ \\
\hline
\end{tabular}

DFT Calculations. Calculations were performed using using the Gaussian 03 suite of programs, revision C. 02. ${ }^{\text {S2 }}$ The geometries were optimized as the RB3LYP/6-31 (d, p) level, and energies were calculated at the same level of theory. Nucleus independent chemical shifts (NICS) were calculated using the gauge invariant atomic orbital (GIAO) approach at the GIAO-B3LYP/6-311+G (d, p) level. 


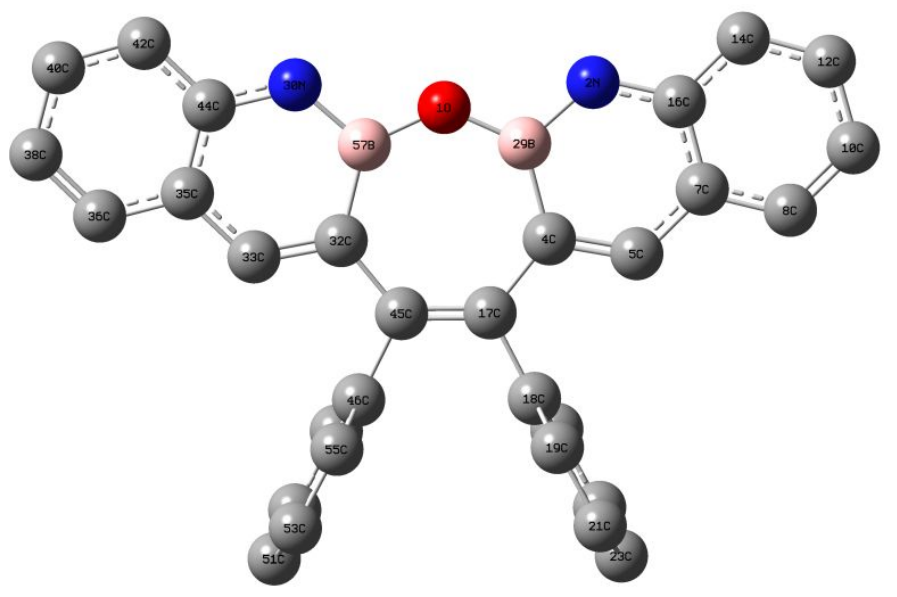

Figure S5. Optimized geometry of 3. Selected bond parameters: 1O-29B 1.37336, 29B-4C 1.54634, 29B-2N $1.43169,4 \mathrm{C}-17 \mathrm{C} 1.48270,17 \mathrm{C}-45 \mathrm{C} 1.37760 ; 57 \mathrm{~B}-1 \mathrm{O}-29 \mathrm{~B} 130.65213,1 \mathrm{O}-29 \mathrm{~B}-4 \mathrm{C} 128.67318,29 \mathrm{~B}-4 \mathrm{C}-17 \mathrm{C}$ $126.99301,4 \mathrm{C}-17 \mathrm{C}-45 \mathrm{C} 129.00763$.

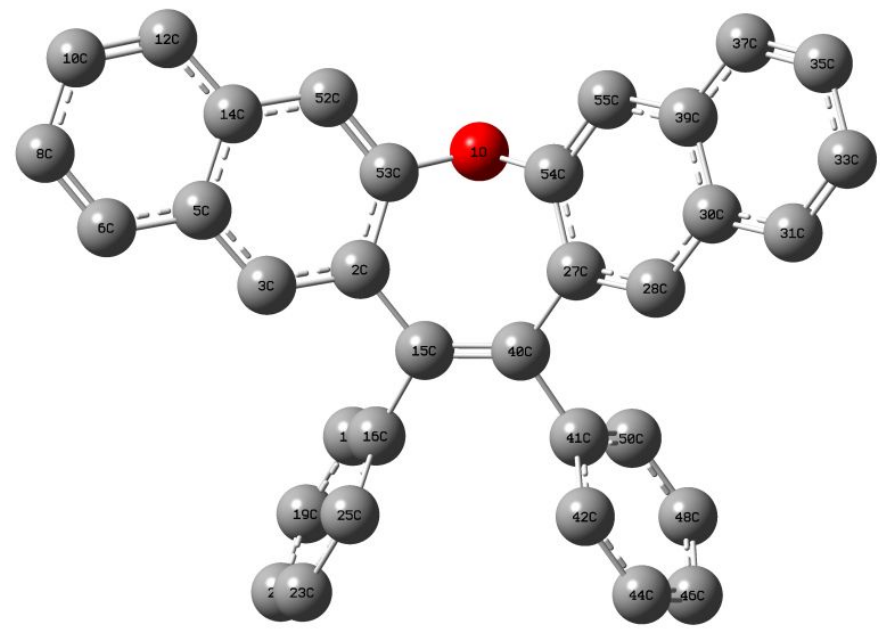

Figure S6. Optimized geometry of 3'. Selected bond parameters: $1 \mathrm{O}-53 \mathrm{C} 1.38743,53 \mathrm{C}-2 \mathrm{C} 1.42749,53 \mathrm{C}-52 \mathrm{C}$ $1.37304, \quad 2 \mathrm{C}-15 \mathrm{C} \quad 1.48566, \quad 15 \mathrm{C}-40 \mathrm{C} \quad 1.36256 ; \quad 53 \mathrm{C}-1 \mathrm{O}-54 \mathrm{C} \quad 115.04307, \quad 1 \mathrm{O}-54 \mathrm{C}-27 \mathrm{C} \quad 119.86357$, $54 \mathrm{C}-27 \mathrm{C}-40 \mathrm{C} 122.31577,27 \mathrm{C}-40 \mathrm{C}-15 \mathrm{C} 125.04441$.

Table S2. Cartesian coordinates for $\mathbf{3}$

\begin{tabular}{lllll}
\hline Tag & Symbol & $\mathrm{X}$ & $\mathrm{Y}$ & $\mathrm{Z}$ \\
\hline 1 & $\mathrm{O}$ & 0.00001746 & 2.56461256 & -0.00000741 \\
2 & $\mathrm{~N}$ & 2.32122754 & 2.93885888 & 0.00001905 \\
3 & $\mathrm{H}$ & 2.08971778 & 3.92457851 & 0.00001799 \\
4 & $\mathrm{C}$ & 1.62205474 & 0.49087857 & 0.00001058 \\
5 & $\mathrm{C}$ & 2.98207996 & 0.24719245 & 0.00004604 \\
6 & $\mathrm{H}$ & 3.34714453 & -0.77496526 & 0.00006033 \\
7 & $\mathrm{C}$ & 4.01095029 & 1.2512613 & 0.00006879
\end{tabular}




\begin{tabular}{|c|c|c|c|c|}
\hline 8 & $\mathrm{C}$ & 5.37996993 & 0.90746648 & 0.00010531 \\
\hline 9 & $\mathrm{H}$ & 5.64867238 & -0.14571319 & 0.00011589 \\
\hline 10 & $\mathrm{C}$ & 6.3667749 & 1.88007416 & 0.00012753 \\
\hline 11 & $\mathrm{H}$ & 7.41448553 & 1.59659867 & 0.00015558 \\
\hline 12 & $\mathrm{C}$ & 6.00391224 & 3.23696294 & 0.00011324 \\
\hline 13 & $\mathrm{H}$ & 6.77278774 & 4.00402759 & 0.00013124 \\
\hline 14 & $\mathrm{C}$ & 4.66682951 & 3.6089428 & 0.00007726 \\
\hline 15 & $\mathrm{H}$ & 4.38552417 & 4.65925857 & 0.00006615 \\
\hline 16 & $\mathrm{C}$ & 3.66314854 & 2.62602781 & 0.00005503 \\
\hline 17 & $\mathrm{C}$ & 0.68880304 & -0.66126201 & -0.00000476 \\
\hline 18 & $\mathrm{C}$ & 1.36933292 & -2.01085185 & -0.00003069 \\
\hline 19 & $\mathrm{C}$ & 1.71732781 & -2.6393054 & 1.20386501 \\
\hline 20 & $\mathrm{H}$ & 1.46757732 & -2.15798905 & 2.14509936 \\
\hline 21 & $\mathrm{C}$ & 2.37461729 & -3.8702119 & 1.20542873 \\
\hline 22 & $\mathrm{H}$ & 2.62899597 & -4.34359957 & 2.14968626 \\
\hline 23 & $\mathrm{C}$ & 2.70416295 & -4.49124267 & -0.00009737 \\
\hline 24 & $\mathrm{H}$ & 3.21480788 & -5.44991076 & -0.00012383 \\
\hline 25 & $\mathrm{C}$ & 2.37474852 & -3.87007696 & -1.20559172 \\
\hline 26 & $\mathrm{H}$ & 2.62922933 & -4.34336039 & -2.14987412 \\
\hline 27 & $\mathrm{C}$ & 1.71746064 & -2.63917099 & -1.20396079 \\
\hline 28 & $\mathrm{H}$ & 1.46781492 & -2.15774563 & -2.14516762 \\
\hline 29 & $\mathrm{~B}$ & 1.24798834 & 1.99129081 & 0.00000318 \\
\hline 30 & $\mathrm{~N}$ & -2.32119102 & 2.93887793 & -0.00003122 \\
\hline 31 & $\mathrm{H}$ & -2.08967439 & 3.92459597 & -0.00003281 \\
\hline 32 & $\mathrm{C}$ & -1.62203728 & 0.49089498 & -0.00001852 \\
\hline 33 & $\mathrm{C}$ & -2.98206197 & 0.24721574 & -0.00004763 \\
\hline 34 & $\mathrm{H}$ & -3.34713077 & -0.77494145 & -0.00005556 \\
\hline 35 & $\mathrm{C}$ & -4.01092595 & 1.25129146 & -0.00007038 \\
\hline 36 & $\mathrm{C}$ & -5.37994824 & 0.90750511 & -0.00010107 \\
\hline 37 & $\mathrm{H}$ & -5.64865756 & -0.14567244 & -0.00010689 \\
\hline 38 & $\mathrm{C}$ & -6.36674633 & 1.8801202 & -0.00012277 \\
\hline 39 & $\mathrm{H}$ & -7.41445907 & 1.59665159 & -0.00014605 \\
\hline 40 & $\mathrm{C}$ & -6.00387414 & 3.23700634 & -0.00011377 \\
\hline 41 & $\mathrm{H}$ & -6.77274487 & 4.00407628 & -0.00013124 \\
\hline 42 & $\mathrm{C}$ & -4.66678929 & 3.6089772 & -0.00008361 \\
\hline 43 & $\mathrm{H}$ & -4.38547655 & 4.65929085 & -0.0000762 \\
\hline 44 & $\mathrm{C}$ & -3.66311467 & 2.62605586 & -0.00006244 \\
\hline 45 & $\mathrm{C}$ & -0.68879986 & -0.66125301 & 0.00000318 \\
\hline 46 & $\mathrm{C}$ & -1.36935461 & -2.01083015 & 0.0000344 \\
\hline 47 & $\mathrm{C}$ & -1.71734686 & -2.63928793 & -1.20385919 \\
\hline 48 & $\mathrm{H}$ & -1.46757203 & -2.15798747 & -2.14509565 \\
\hline 49 & $\mathrm{C}$ & -2.37466068 & -3.8701812 & -1.20541973 \\
\hline 50 & $\mathrm{H}$ & -2.6290346 & -4.3435747 & -2.14967568 \\
\hline 51 & $\mathrm{C}$ & -2.70423756 & -4.49119187 & 0.00010901 \\
\hline
\end{tabular}




\begin{tabular}{lllll}
52 & $\mathrm{H}$ & -3.21490207 & -5.44984991 & 0.00013812 \\
53 & $\mathrm{C}$ & -2.37483107 & -3.87001822 & 1.20560124 \\
54 & $\mathrm{H}$ & -2.62933781 & -4.34328471 & 2.14988471 \\
55 & $\mathrm{C}$ & -1.71751832 & -2.63912548 & 1.20396608 \\
56 & $\mathrm{H}$ & -1.46787948 & -2.15769324 & 2.14517185 \\
57 & $\mathrm{~B}$ & -1.24795818 & 1.99130245 & -0.00001693 \\
\hline
\end{tabular}

Table S3. Cartesian coordinates for 3'

\begin{tabular}{|c|c|c|c|c|}
\hline Tag & Symbol & $\mathrm{X}$ & $\mathrm{Y}$ & $\mathrm{Z}$ \\
\hline 1 & $\mathrm{O}$ & -0.00001852 & -1.52935294 & 1.60145599 \\
\hline 2 & $\mathrm{C}$ & -1.53437219 & -0.34725511 & 0.12380948 \\
\hline 3 & $\mathrm{C}$ & -2.76358902 & -0.36647271 & -0.52117873 \\
\hline 4 & $\mathrm{H}$ & -3.08768786 & 0.51287436 & -1.06795483 \\
\hline 5 & $\mathrm{C}$ & -3.62624425 & -1.48918786 & -0.48905821 \\
\hline 6 & $\mathrm{C}$ & -4.87718593 & -1.49940574 & -1.16458471 \\
\hline 7 & $\mathrm{H}$ & -5.17335585 & -0.61890613 & -1.72865644 \\
\hline 8 & $\mathrm{C}$ & -5.69936174 & -2.60130782 & -1.10644718 \\
\hline 9 & $\mathrm{H}$ & -6.65236446 & -2.5984 & -1.6270433 \\
\hline 10 & $\mathrm{C}$ & -5.30895276 & -3.74490061 & -0.36630337 \\
\hline 11 & $\mathrm{H}$ & -5.96666928 & -4.60831732 & -0.32749139 \\
\hline 12 & $\mathrm{C}$ & -4.10505758 & -3.76611003 & 0.30156171 \\
\hline 13 & $\mathrm{H}$ & -3.80507388 & -4.64229473 & 0.87017483 \\
\hline 14 & $\mathrm{C}$ & -3.23351165 & -2.64596245 & 0.25744844 \\
\hline 15 & $\mathrm{C}$ & -0.68127761 & 0.86869631 & 0.09406284 \\
\hline 16 & $\mathrm{C}$ & -1.44338598 & 2.16485195 & 0.06434054 \\
\hline 17 & $\mathrm{C}$ & -1.98431414 & 2.67562865 & 1.25342299 \\
\hline 18 & $\mathrm{H}$ & -1.82427665 & 2.13714318 & 2.18299638 \\
\hline 19 & $\mathrm{C}$ & -2.71437607 & 3.86368132 & 1.25247047 \\
\hline 20 & $\mathrm{H}$ & -3.11643965 & 4.2491118 & 2.18508028 \\
\hline 21 & $\mathrm{C}$ & -2.93068574 & 4.55372369 & 0.05830422 \\
\hline 22 & $\mathrm{H}$ & -3.50204529 & 5.47736024 & 0.056541 \\
\hline 23 & $\mathrm{C}$ & -2.41049603 & 4.04807101 & -1.13303199 \\
\hline 24 & $\mathrm{H}$ & -2.57618196 & 4.57588775 & -2.06793526 \\
\hline 25 & $\mathrm{C}$ & -1.67176777 & 2.86353896 & -1.12928118 \\
\hline 26 & $\mathrm{H}$ & -1.27042863 & 2.47420897 & -2.05998543 \\
\hline 27 & $\mathrm{C}$ & 1.5343542 & -0.34727998 & 0.12381319 \\
\hline 28 & $\mathrm{C}$ & 2.76357156 & -0.36651028 & -0.5211708 \\
\hline 29 & $\mathrm{H}$ & 3.08768098 & 0.51283626 & -1.06794372 \\
\hline 30 & $\mathrm{C}$ & 3.62621197 & -1.48923601 & -0.48905027 \\
\hline 31 & $\mathrm{C}$ & 4.87715524 & -1.49946766 & -1.16457413 \\
\hline 32 & $\mathrm{H}$ & 5.17333786 & -0.61897069 & -1.72864321 \\
\hline 33 & $\mathrm{C}$ & 5.69931676 & -2.60138085 & -1.1064366 \\
\hline 34 & $\mathrm{H}$ & 6.65232054 & -2.59848361 & -1.6270306 \\
\hline 35 & $\mathrm{C}$ & 5.30889084 & -3.74496993 & -0.36629649 \\
\hline
\end{tabular}




\begin{tabular}{lllll}
36 & $\mathrm{H}$ & 5.96659678 & -4.60839511 & -0.32748452 \\
37 & $\mathrm{C}$ & 4.10499408 & -3.76616559 & 0.30156594 \\
38 & $\mathrm{H}$ & 3.80499821 & -4.64234818 & 0.87017642 \\
39 & $\mathrm{C}$ & 3.23346244 & -2.6460069 & 0.2574532 \\
40 & $\mathrm{C}$ & 0.6812829 & 0.86868414 & 0.09406919 \\
41 & $\mathrm{C}$ & 1.44342091 & 2.16482126 & 0.06433737 \\
42 & $\mathrm{C}$ & 1.67183762 & 2.86348446 & -1.12929177 \\
43 & $\mathrm{H}$ & 1.27050377 & 2.47415024 & -2.05999548 \\
44 & $\mathrm{C}$ & 2.41059393 & 4.04799905 & -1.13304893 \\
45 & $\mathrm{H}$ & 2.57630473 & 4.57579832 & -2.06795695 \\
46 & $\mathrm{C}$ & 2.93078152 & 4.55365437 & 0.05828729 \\
47 & $\mathrm{H}$ & 3.50216436 & 5.47727716 & 0.05651878 \\
48 & $\mathrm{C}$ & 2.7144438 & 3.86363211 & 1.25245989 \\
49 & $\mathrm{H}$ & 3.11650739 & 4.24906365 & 2.18506917 \\
50 & $\mathrm{C}$ & 1.98435383 & 2.67559637 & 1.25341876 \\
51 & $\mathrm{H}$ & 1.8242957 & 2.13712518 & 2.18299638 \\
52 & $\mathrm{C}$ & -1.98328489 & -2.62149329 & 0.92518546 \\
53 & $\mathrm{C}$ & -1.17044112 & -1.5170374 & 0.85653317 \\
54 & $\mathrm{C}$ & 1.17040513 & -1.51705645 & 0.85653529 \\
55 & $\mathrm{C}$ & 1.98323409 & -2.62152345 & 0.92518757 \\
56 & $\mathrm{H}$ & -1.65882621 & -3.4729379 & 1.51492334 \\
\hline & $\mathrm{H}$ & 1.65876324 & -3.47296436 & 1.51492387 \\
\hline
\end{tabular}

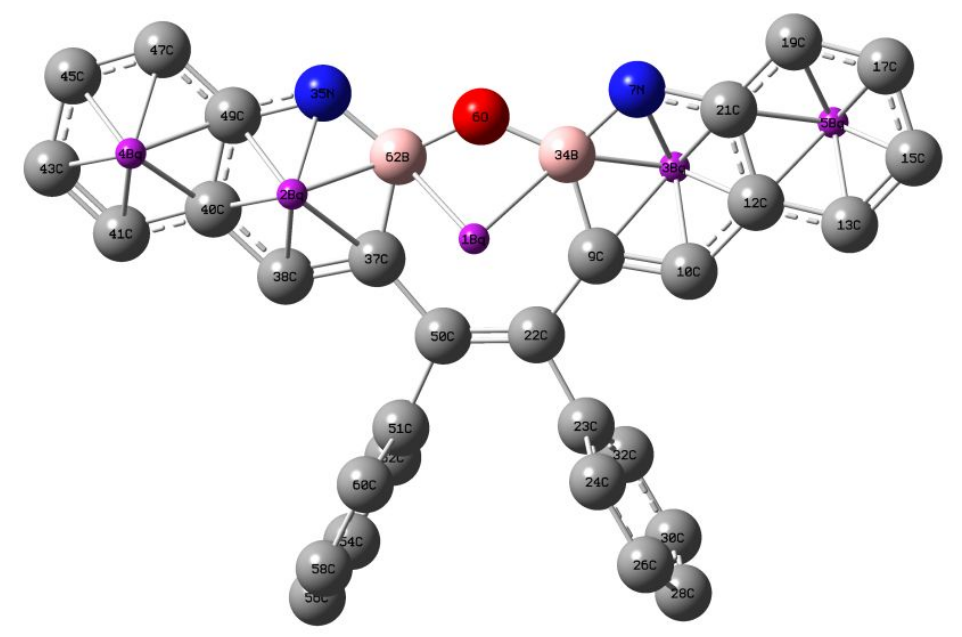

$1 \mathrm{~Bq}$ Isotropic $=-3.4884, \mathrm{NICS}(1)=3.4884,2 \mathrm{~Bq}, 3 \mathrm{~Bq}$ Isotropic $=3.6476, \mathrm{NICS}(1)=-3.6476,4 \mathrm{~Bq}, 5 \mathrm{~Bq}$ Isotropic $=9.1886, \operatorname{NICS}(1)=-9.1886$.

Figure S7. NICS(1)zz values (in ppm) of $\mathbf{3}$ calculated at the GIAO-B3LYP16-311+G (d, p) level.

Table S4. Cartesian coordinates for NICS calculations on 3

\begin{tabular}{lllll}
\hline Tag & Symbol & $\mathrm{X}$ & $\mathrm{Y}$ & $\mathrm{Z}$ \\
\hline 1 & $\mathrm{~Bq}$ & -0.000009 & 0.930002 & 1.000000 \\
2 & $\mathrm{~Bq}$ & -2.660022 & 1.619999 & 1.000000
\end{tabular}




\begin{tabular}{|c|c|c|c|c|}
\hline 3 & $\mathrm{~Bq}$ & 2.650026 & 1.619996 & -1.000000 \\
\hline 4 & $\mathrm{~Bq}$ & -5.020022 & 2.249999 & 1.000000 \\
\hline 5 & $\mathrm{~Bq}$ & 5.020026 & 2.249996 & -1.000000 \\
\hline 6 & $\mathrm{O}$ & 0.000018 & 2.564612 & -0.000007 \\
\hline 7 & $\mathrm{~N}$ & 2.321228 & 2.938859 & 0.000019 \\
\hline 8 & $\mathrm{H}$ & 2.089718 & 3.924578 & 0.000018 \\
\hline 9 & $\mathrm{C}$ & 1.622055 & 0.490879 & 0.000011 \\
\hline 10 & $\mathrm{C}$ & 2.98208 & 0.247192 & 0.000046 \\
\hline 11 & $\mathrm{H}$ & 3.347144 & -0.774966 & 0.00006 \\
\hline 12 & $\mathrm{C}$ & 4.01095 & 1.251262 & 0.000069 \\
\hline 13 & $\mathrm{C}$ & 5.37997 & 0.907466 & 0.000105 \\
\hline 14 & $\mathrm{H}$ & 5.648672 & -0.145713 & 0.000116 \\
\hline 15 & $\mathrm{C}$ & 6.366775 & 1.880074 & 0.000128 \\
\hline 16 & $\mathrm{H}$ & 7.414486 & 1.596598 & 0.000156 \\
\hline 17 & $\mathrm{C}$ & 6.003912 & 3.236963 & 0.000113 \\
\hline 18 & $\mathrm{H}$ & 6.772788 & 4.004028 & 0.000131 \\
\hline 19 & $\mathrm{C}$ & 4.66683 & 3.608943 & 0.000077 \\
\hline 20 & $\mathrm{H}$ & 4.385524 & 4.659258 & 0.000066 \\
\hline 21 & $\mathrm{C}$ & 3.663149 & 2.626028 & 0.000055 \\
\hline 22 & $\mathrm{C}$ & 0.688803 & -0.661262 & -0.000005 \\
\hline 23 & $\mathrm{C}$ & 1.369333 & -2.010852 & -0.000031 \\
\hline 24 & $\mathrm{C}$ & 1.717328 & -2.639305 & 1.203865 \\
\hline 25 & $\mathrm{H}$ & 1.467577 & -2.157989 & 2.1451 \\
\hline 26 & $\mathrm{C}$ & 2.374617 & -3.870212 & 1.205429 \\
\hline 27 & $\mathrm{H}$ & 2.628996 & -4.343599 & 2.149686 \\
\hline 28 & $\mathrm{C}$ & 2.704163 & -4.491242 & -0.000097 \\
\hline 29 & $\mathrm{H}$ & 3.214808 & -5.449911 & -0.000124 \\
\hline 30 & $\mathrm{C}$ & 2.374748 & -3.870077 & -1.205592 \\
\hline 31 & $\mathrm{H}$ & 2.629229 & -4.34336 & -2.149874 \\
\hline 32 & $\mathrm{C}$ & 1.717461 & -2.639171 & -1.203961 \\
\hline 33 & $\mathrm{H}$ & 1.467815 & -2.157745 & -2.145168 \\
\hline 34 & B & 1.247988 & 1.991291 & 0.000003 \\
\hline 35 & $\mathrm{~N}$ & -2.321191 & 2.938878 & -0.000031 \\
\hline 36 & $\mathrm{H}$ & -2.089675 & 3.924596 & -0.000033 \\
\hline 37 & $\mathrm{C}$ & -1.622037 & 0.490895 & -0.000018 \\
\hline 38 & $\mathrm{C}$ & -2.982062 & 0.247216 & -0.000048 \\
\hline 39 & $\mathrm{H}$ & -3.347131 & -0.774942 & -0.000055 \\
\hline 40 & $\mathrm{C}$ & -4.010926 & 1.251292 & -0.00007 \\
\hline 41 & $\mathrm{C}$ & -5.379948 & 0.907505 & -0.000101 \\
\hline 42 & $\mathrm{H}$ & -5.648658 & -0.145672 & -0.000107 \\
\hline 43 & $\mathrm{C}$ & -6.366746 & 1.88012 & -0.000123 \\
\hline 44 & $\mathrm{H}$ & -7.414459 & 1.596651 & -0.000146 \\
\hline 45 & $\mathrm{C}$ & -6.003874 & 3.237007 & -0.000114 \\
\hline 46 & $\mathrm{H}$ & -6.772745 & 4.004076 & -0.000131 \\
\hline
\end{tabular}




\begin{tabular}{lllll}
47 & $\mathrm{C}$ & -4.666789 & 3.608977 & -0.000084 \\
48 & $\mathrm{H}$ & -4.385477 & 4.659291 & -0.000076 \\
49 & $\mathrm{C}$ & -3.663115 & 2.626056 & -0.000062 \\
50 & $\mathrm{C}$ & -0.6888 & -0.661253 & 0.000003 \\
51 & $\mathrm{C}$ & -1.369355 & -2.01083 & 0.000035 \\
52 & $\mathrm{C}$ & -1.717347 & -2.639288 & -1.203859 \\
53 & $\mathrm{H}$ & -1.467572 & -2.157987 & -2.145095 \\
54 & $\mathrm{C}$ & -2.374661 & -3.870181 & -1.20542 \\
55 & $\mathrm{H}$ & -2.629034 & -4.343575 & -2.149676 \\
56 & $\mathrm{C}$ & -2.704237 & -4.491192 & 0.000109 \\
57 & $\mathrm{H}$ & -3.214902 & -5.44985 & 0.000138 \\
58 & $\mathrm{C}$ & -2.374831 & -3.870018 & 1.205601 \\
59 & $\mathrm{H}$ & -2.629338 & -4.343285 & 2.149885 \\
60 & $\mathrm{C}$ & -1.717518 & -2.639126 & 1.203966 \\
61 & $\mathrm{H}$ & -1.467879 & -2.157693 & 2.145172 \\
62 & $\mathrm{~B}$ & -1.247958 & 1.991302 & -0.000017 \\
\hline & & & &
\end{tabular}

UV-Vis and FL Studies of Compound 3 -17
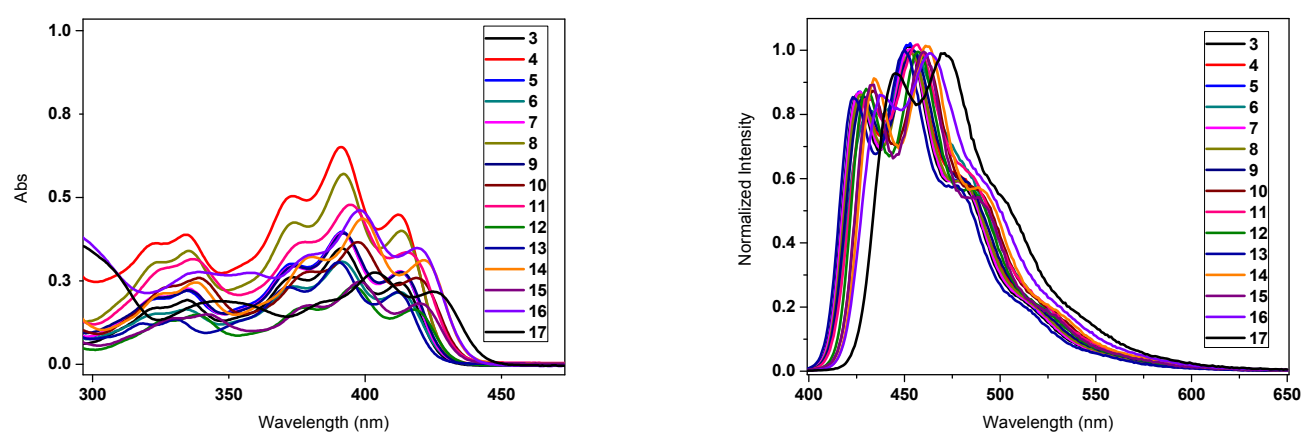

Figure S8. UV-vis spectra of 3-17 in $\mathrm{CH}_{2} \mathrm{Cl}_{2}$ at $10^{-5} \mathrm{M}$ (left) and emission spectra of 3-17 in $\mathrm{CH}_{2} \mathrm{Cl}_{2}$ at $10^{-6} \mathrm{M}$ (right).

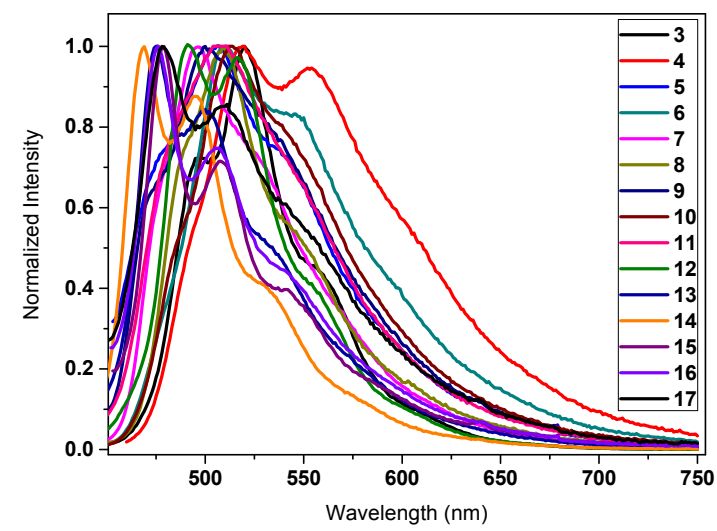

Figure S9. Emission spectra of 3-17 in the Solid State (grinding crystals). 

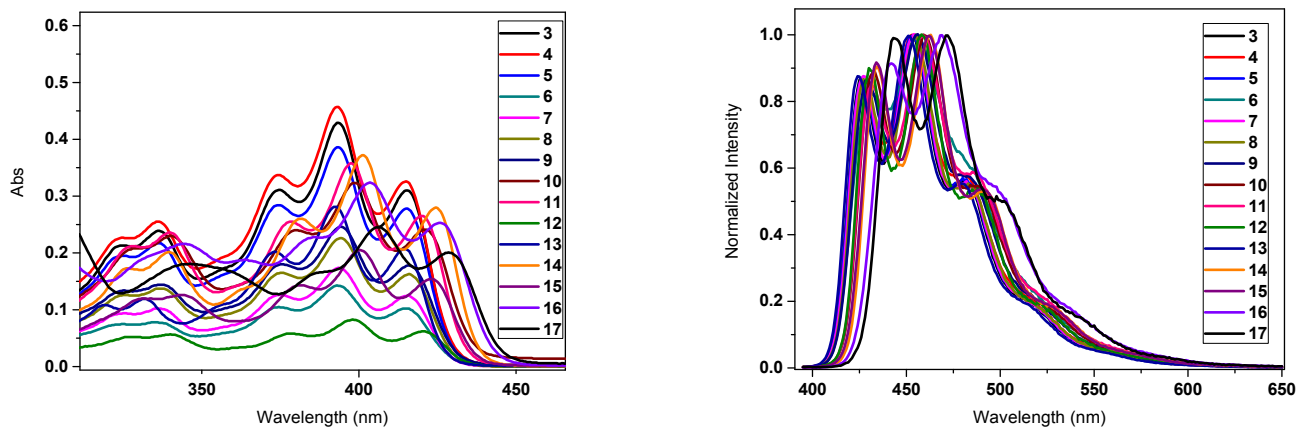

Figure S10. UV-vis spectra of 3-17 in toluene at $10^{-5} \mathrm{M}$ (left) and emission spectra of 3-17 in toluene at $10^{-6} \mathrm{M}$ (right).
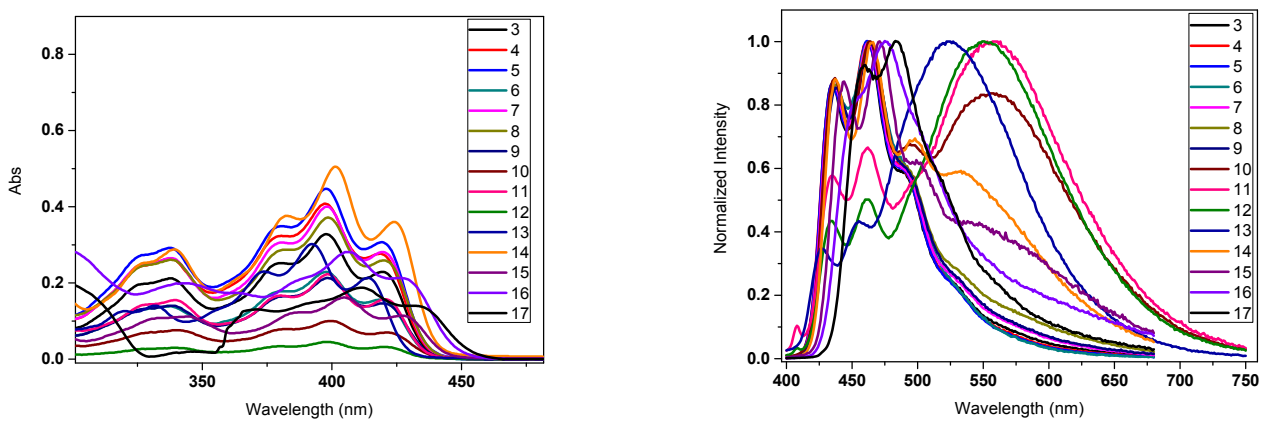

Figure S11. UV-vis spectra of 3-17 in DMSO at $10^{-5} \mathrm{M}$ (left) and emission spectra of 3-17 in DMSO at $10^{-6} \mathrm{M}$ (right).
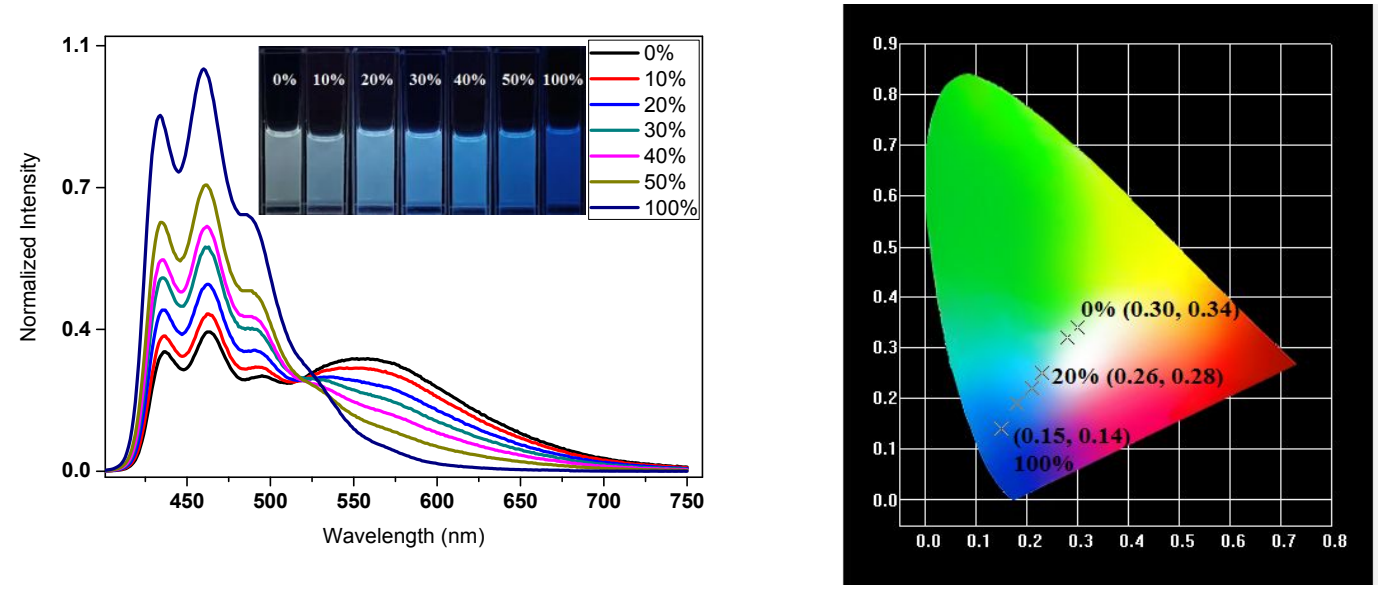

Figure S12. Emission spectra of 10 in DMSO/DCM solution $\left(10^{-6} \mathrm{M}\right)$ at different DCM fractions (left) and calculated PL emission color coordinates in the CIE 1931 chromaticity diagram (right). 

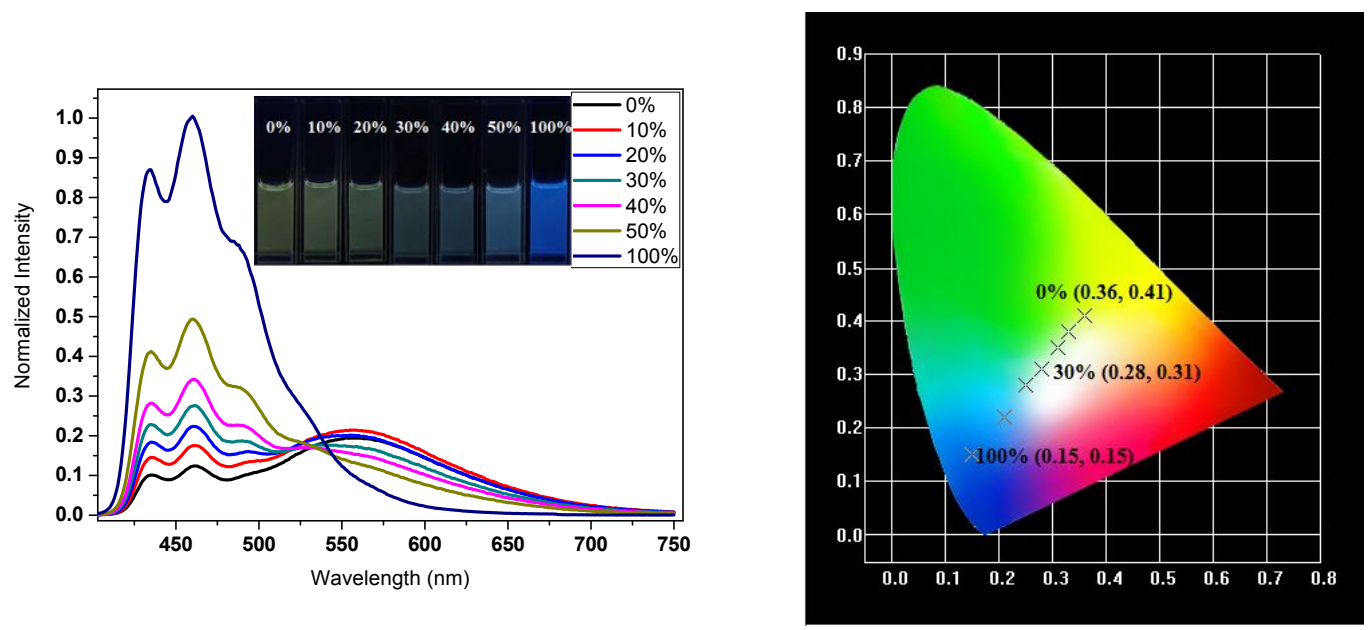

Figure S13. Emission spectra of 11 in DMSO/DCM solution $\left(10^{-6} \mathrm{M}\right)$ at different DCM fractions (left) and calculated PL emission color coordinates in the CIE 1931 chromaticity diagram (right).
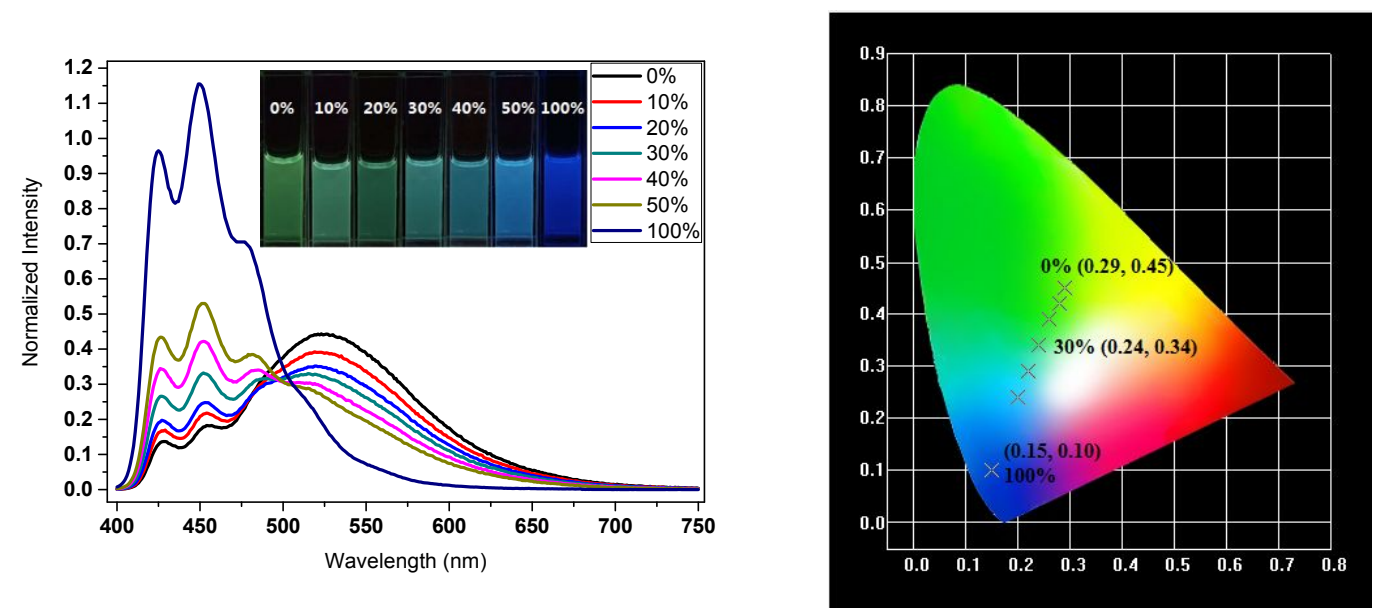

Figure S14. Emission spectra of 13 in DMSO/DCM solution $\left(10^{-6} \mathrm{M}\right)$ at different DCM fractions (left) and calculated PL emission color coordinates in the CIE 1931 chromaticity diagram (right). 
Temperature Dependent ${ }^{1}$ HNMR Studies for 12

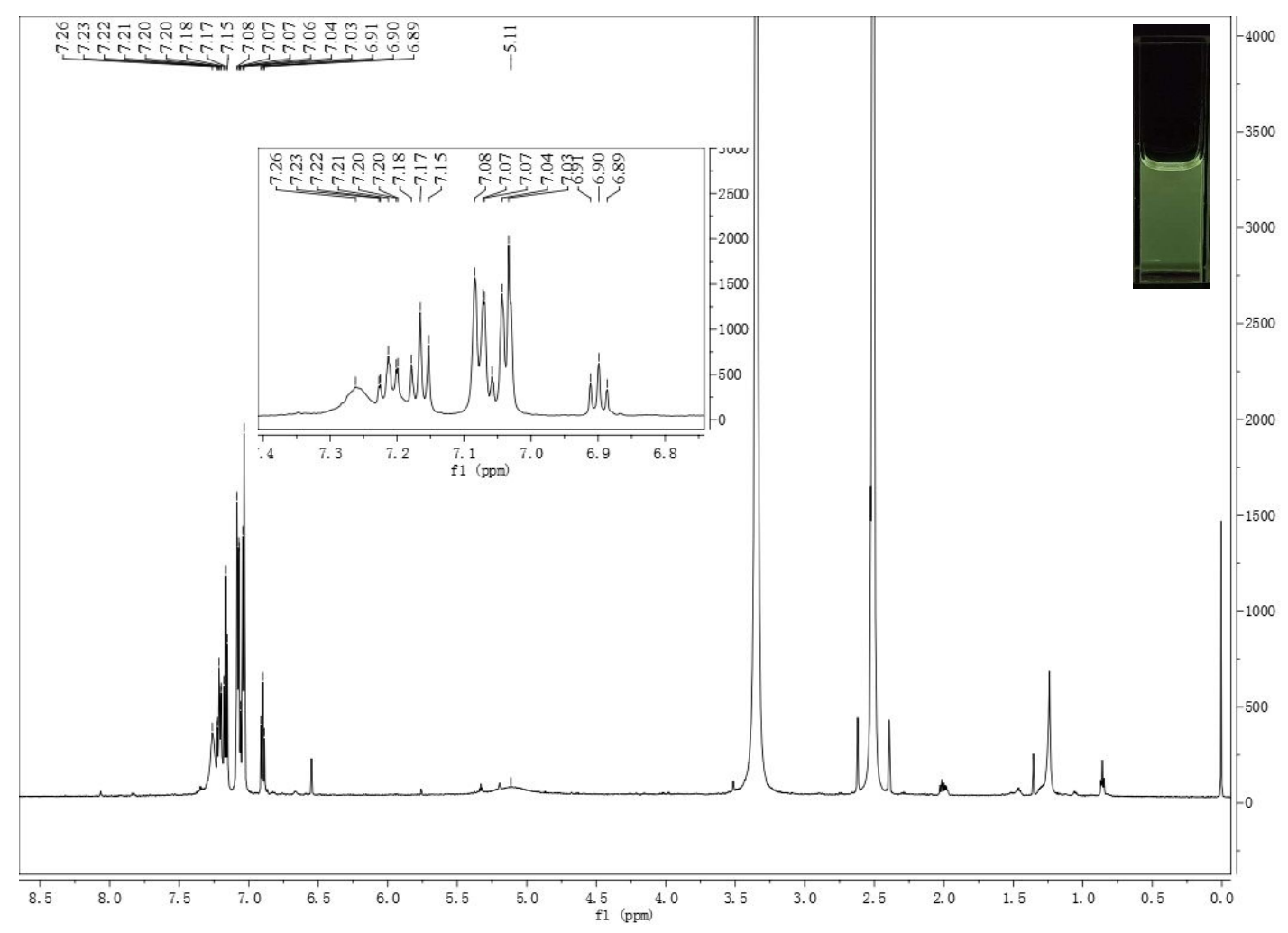

Figure S15. In DMSO- $d_{6}$ at $25^{\circ} \mathrm{C}$.

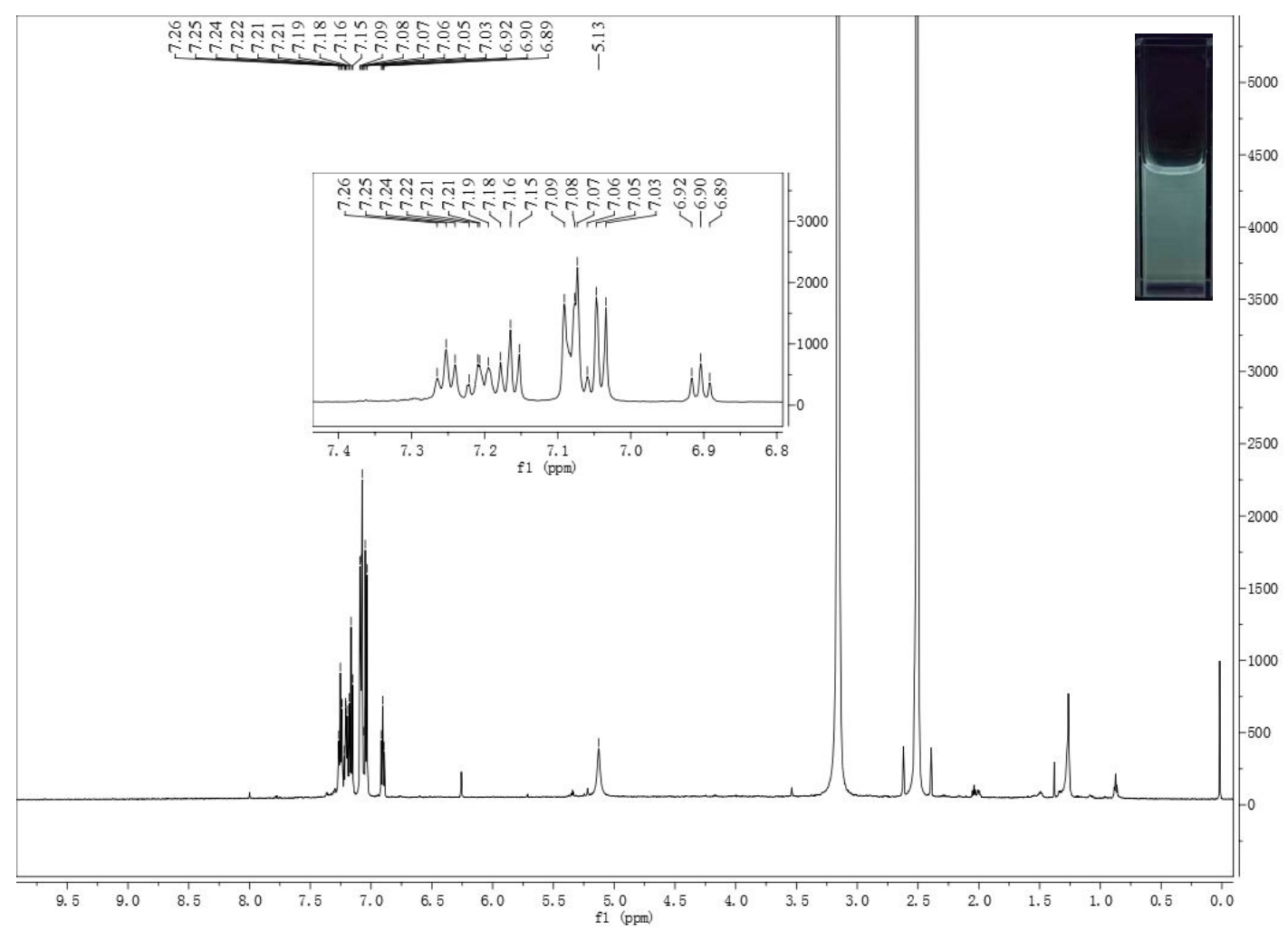

Figure S16. In DMSO- $d_{6}$ at $65^{\circ} \mathrm{C}$. 


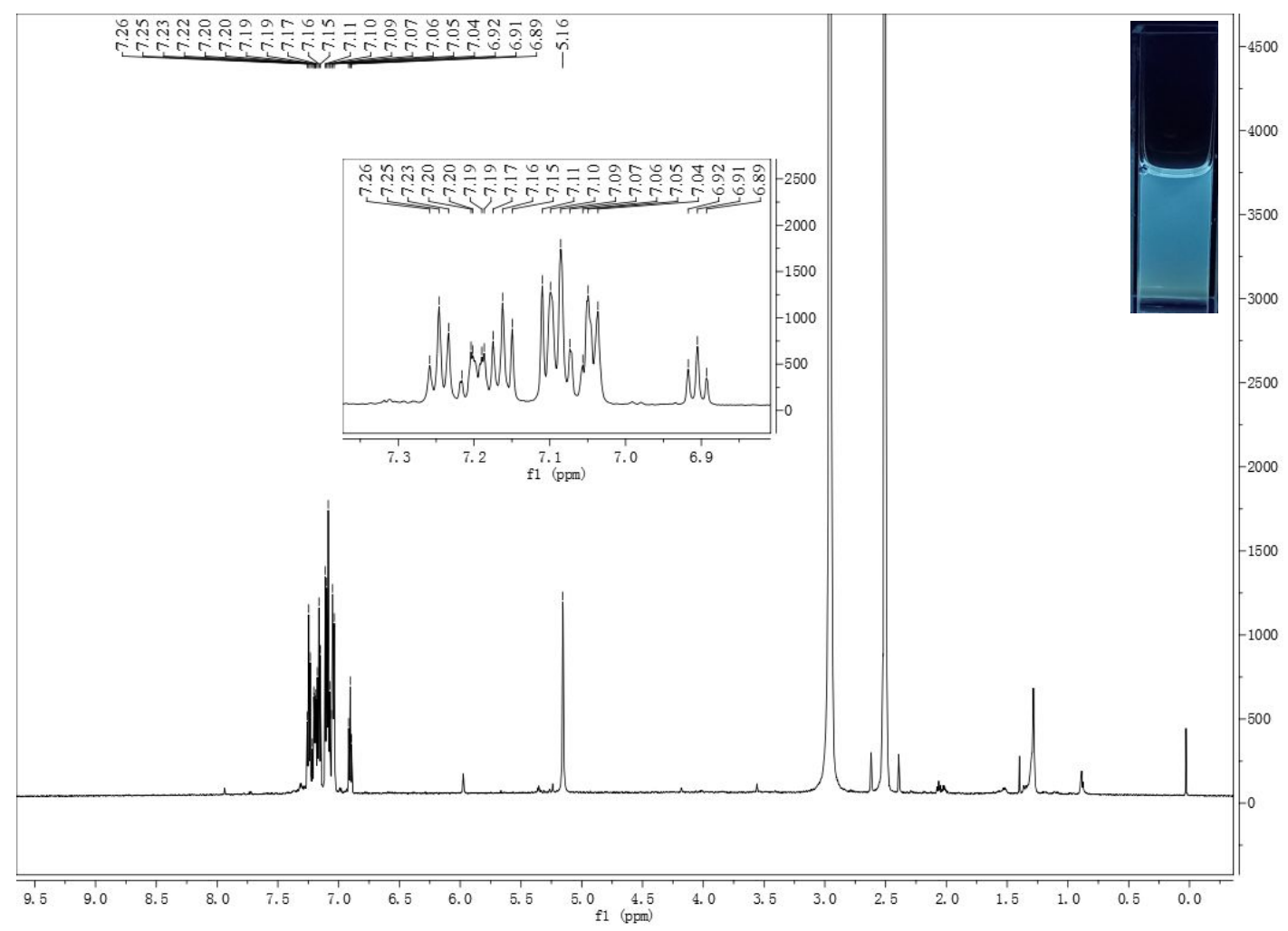

Figure S17. In DMSO- $d_{6}$ at $100{ }^{\circ} \mathrm{C}$.

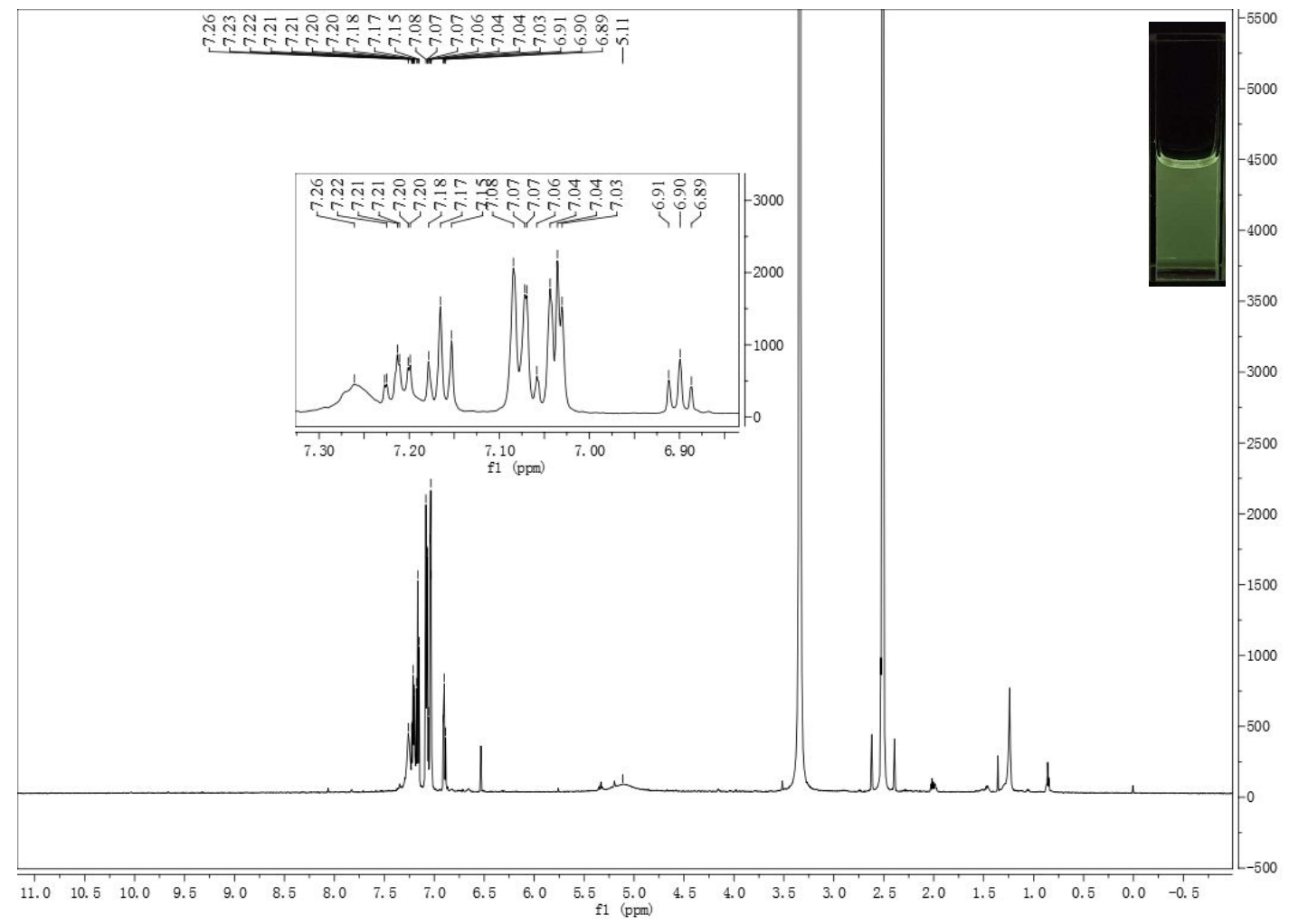

Figure S18. In DMSO- $d_{6}$ retuned to $25^{\circ} \mathrm{C}$. 
Scanned NMR Spectra of All New Compounds

${ }^{1} \mathrm{H}$ - NMR spectrum of $\mathbf{3}$

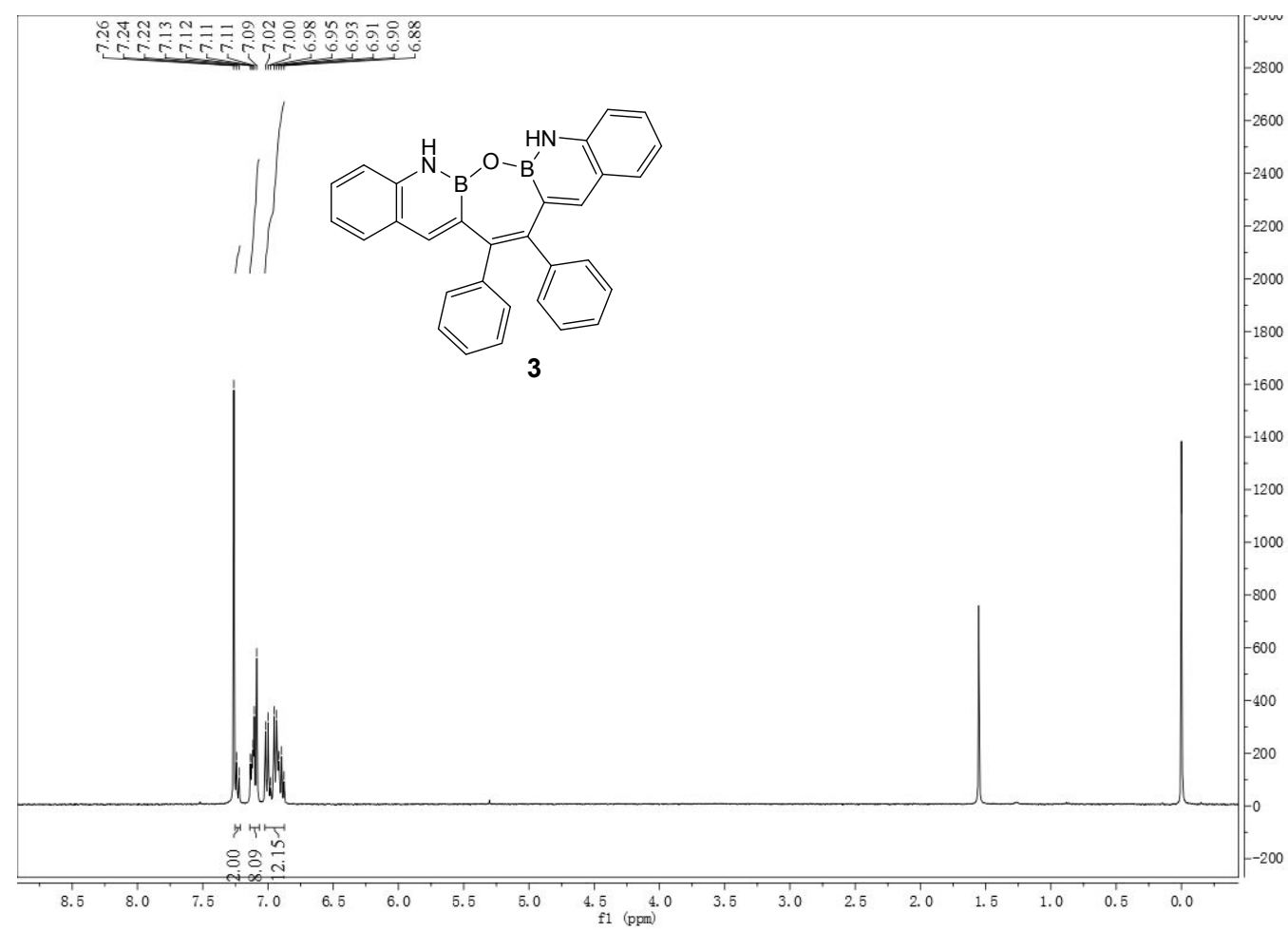

${ }^{13} \mathrm{C}$ - NMR spectrum of $\mathbf{3}$

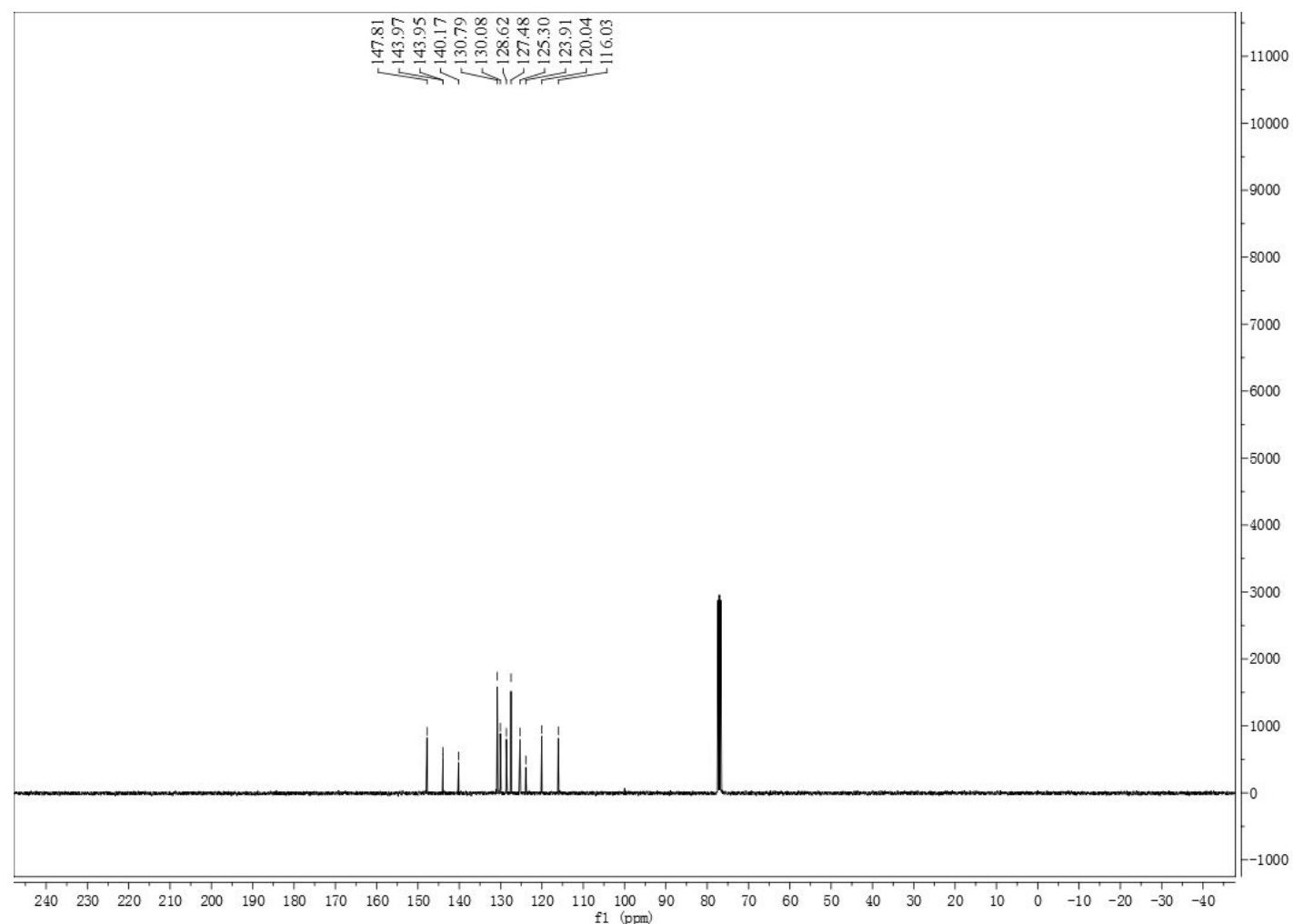


${ }^{11} \mathrm{~B}$ - NMR spectrum of $\mathbf{3}$ (We attribute the multiple peaks in the boron NMRs to the boron in the glass of the nuclear magnetic tube. These signals usually appear at 0 to $-20 \mathrm{ppm}$ and are easy to distinguish from the signals of three coordinated boron.)

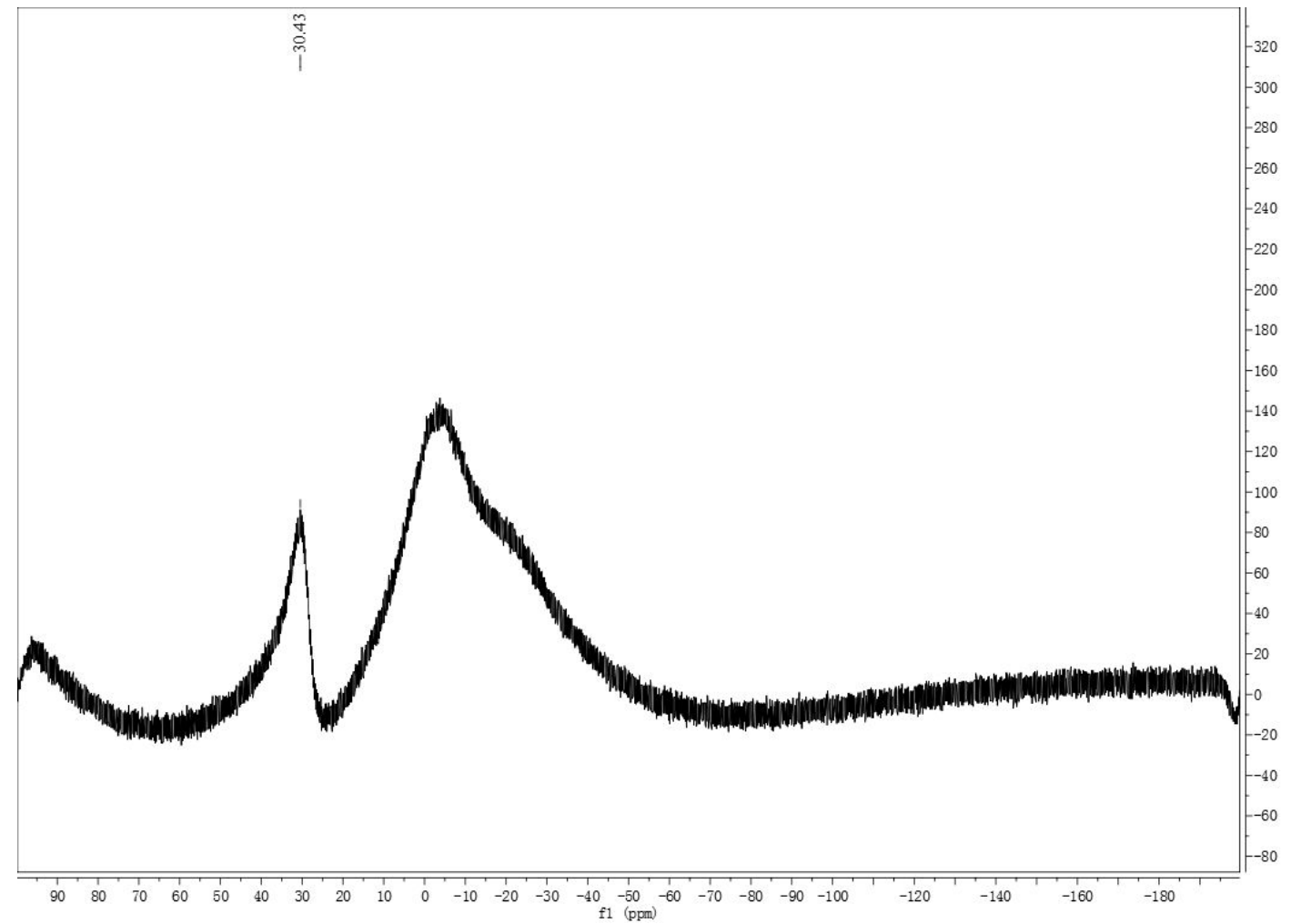

HRMS spectrum of $\mathbf{3}$ 


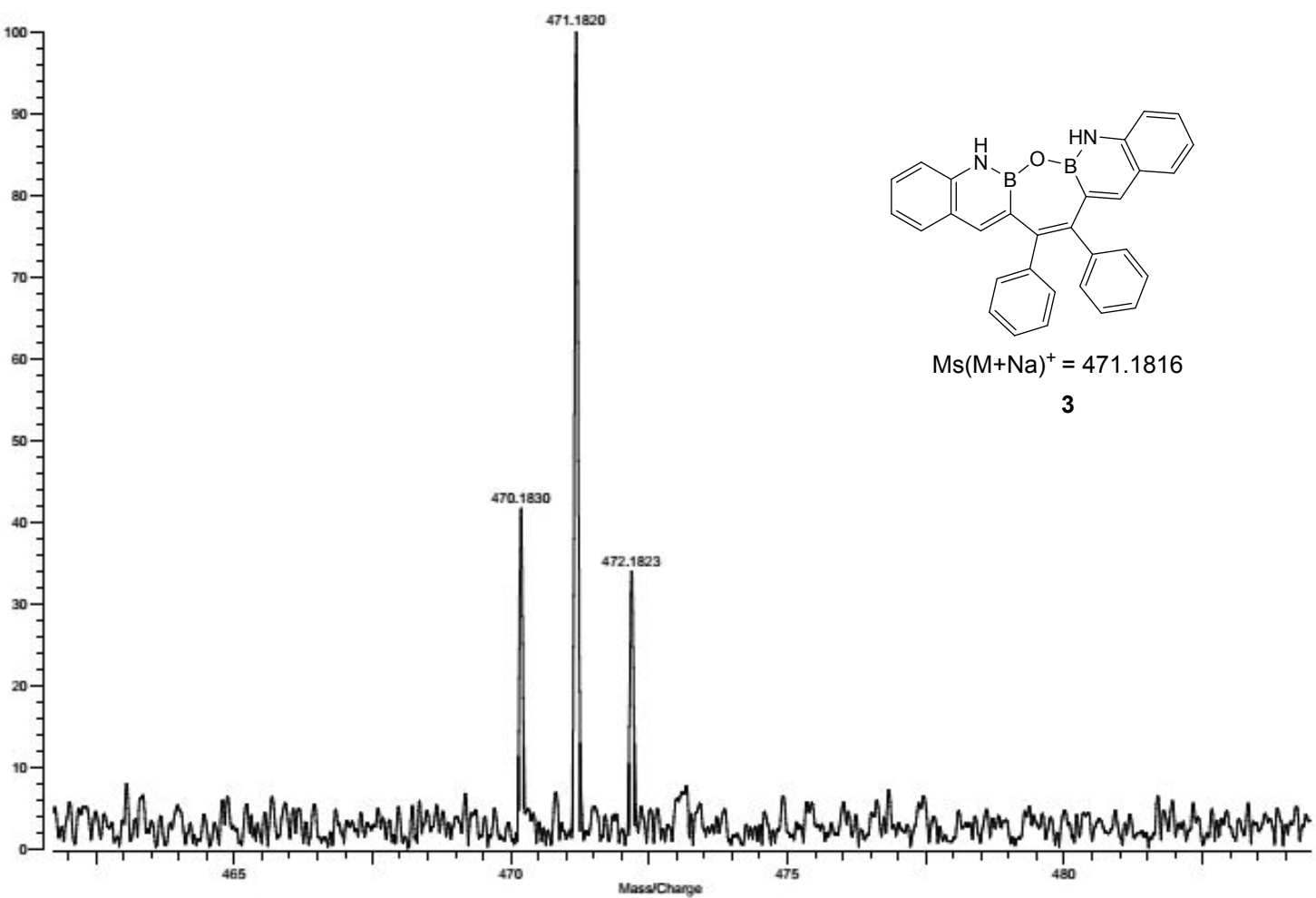

${ }^{1} \mathrm{H}$ - NMR spectrum of $\mathbf{4}$

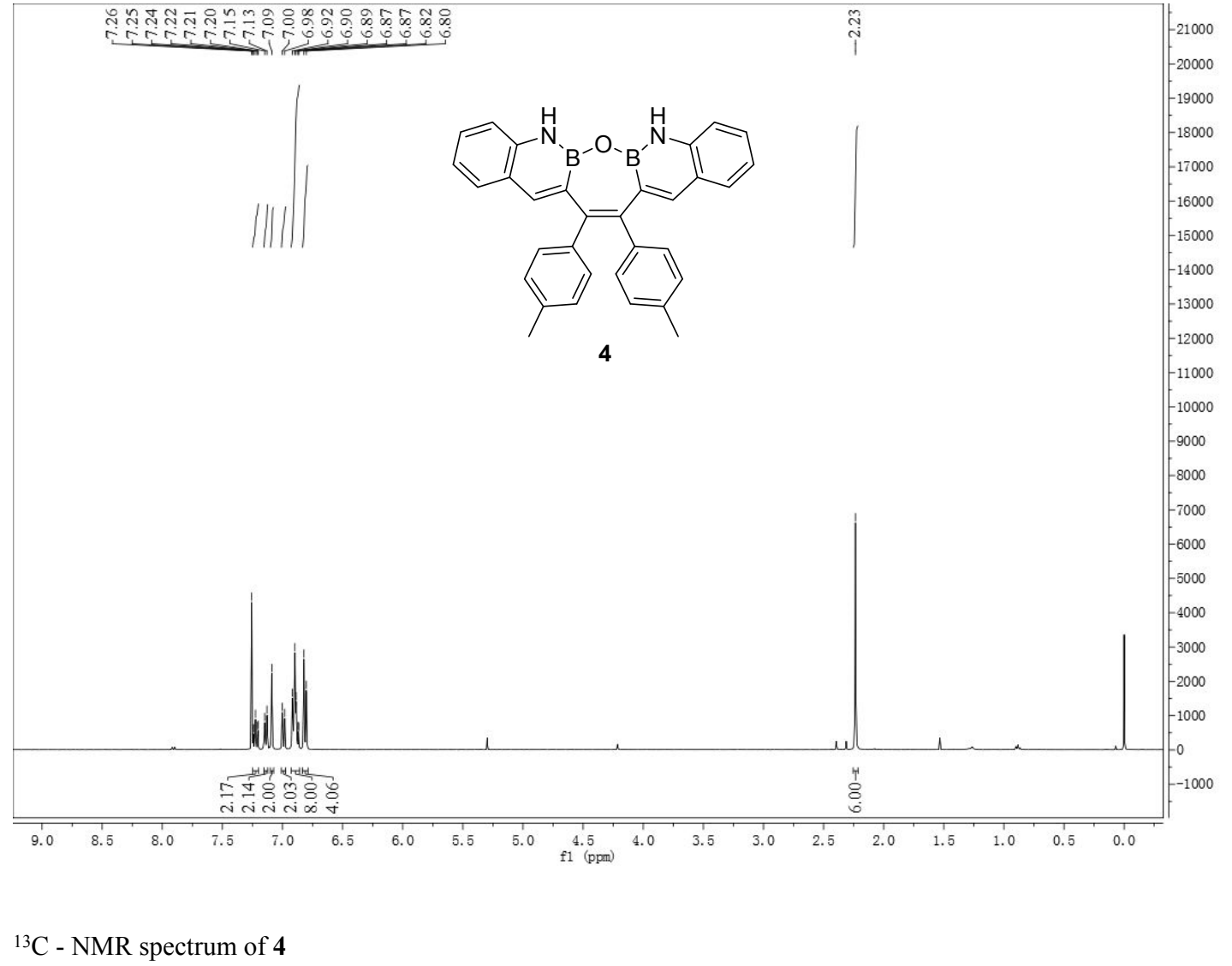




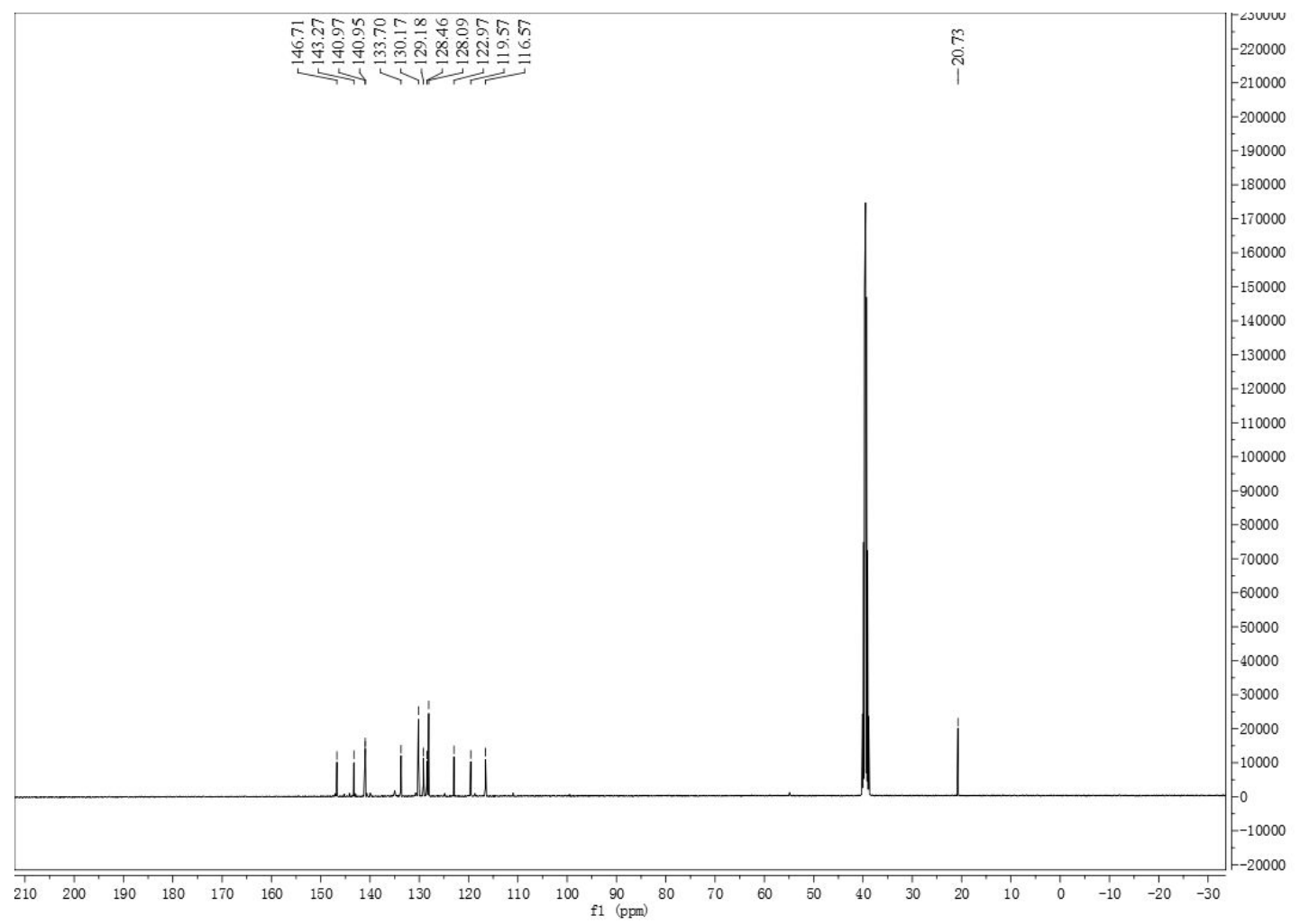

${ }^{11} \mathrm{~B}-\mathrm{NMR}$ spectrum of $\mathbf{4}$

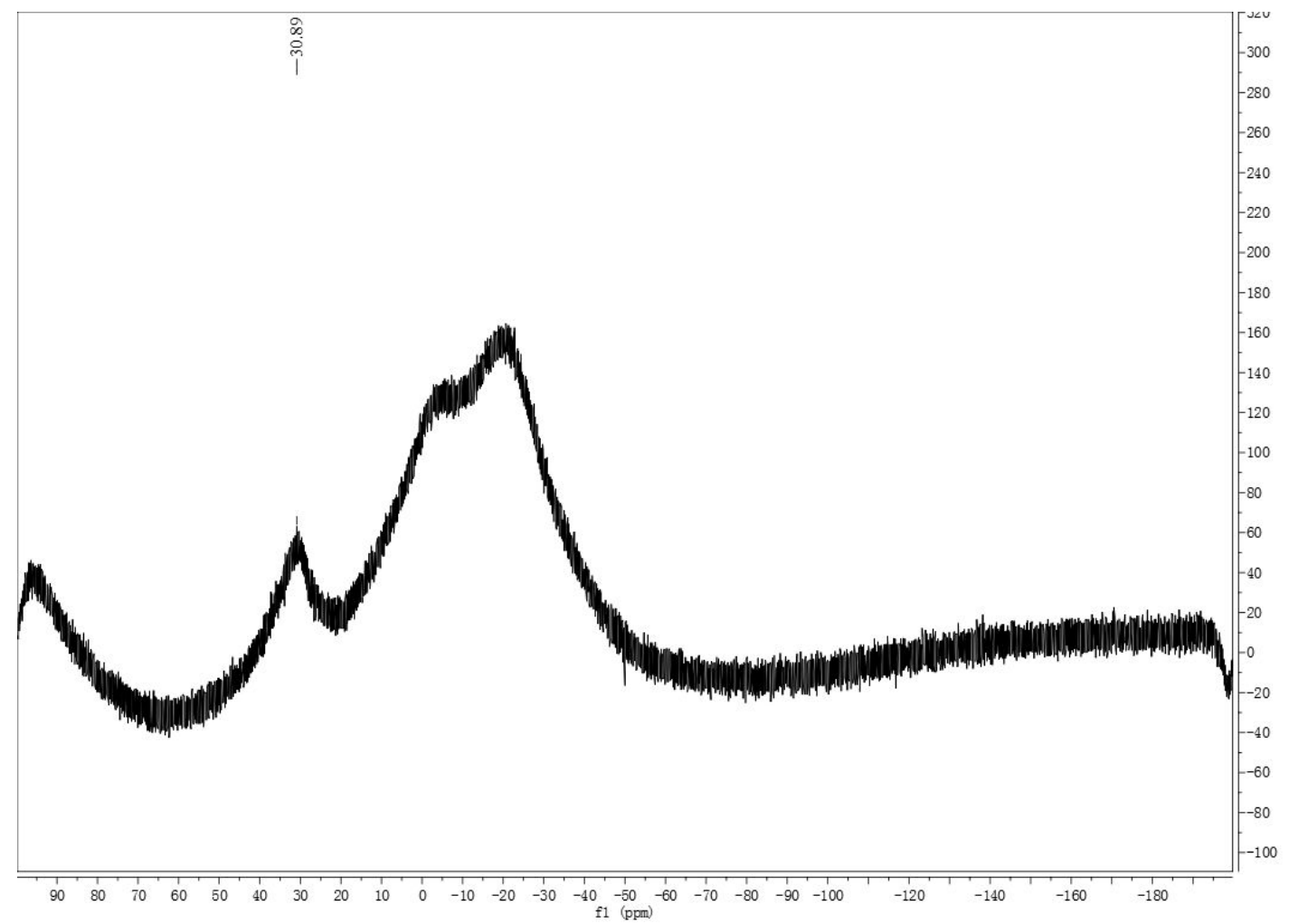

HRMS spectrum of $\mathbf{4}$ 


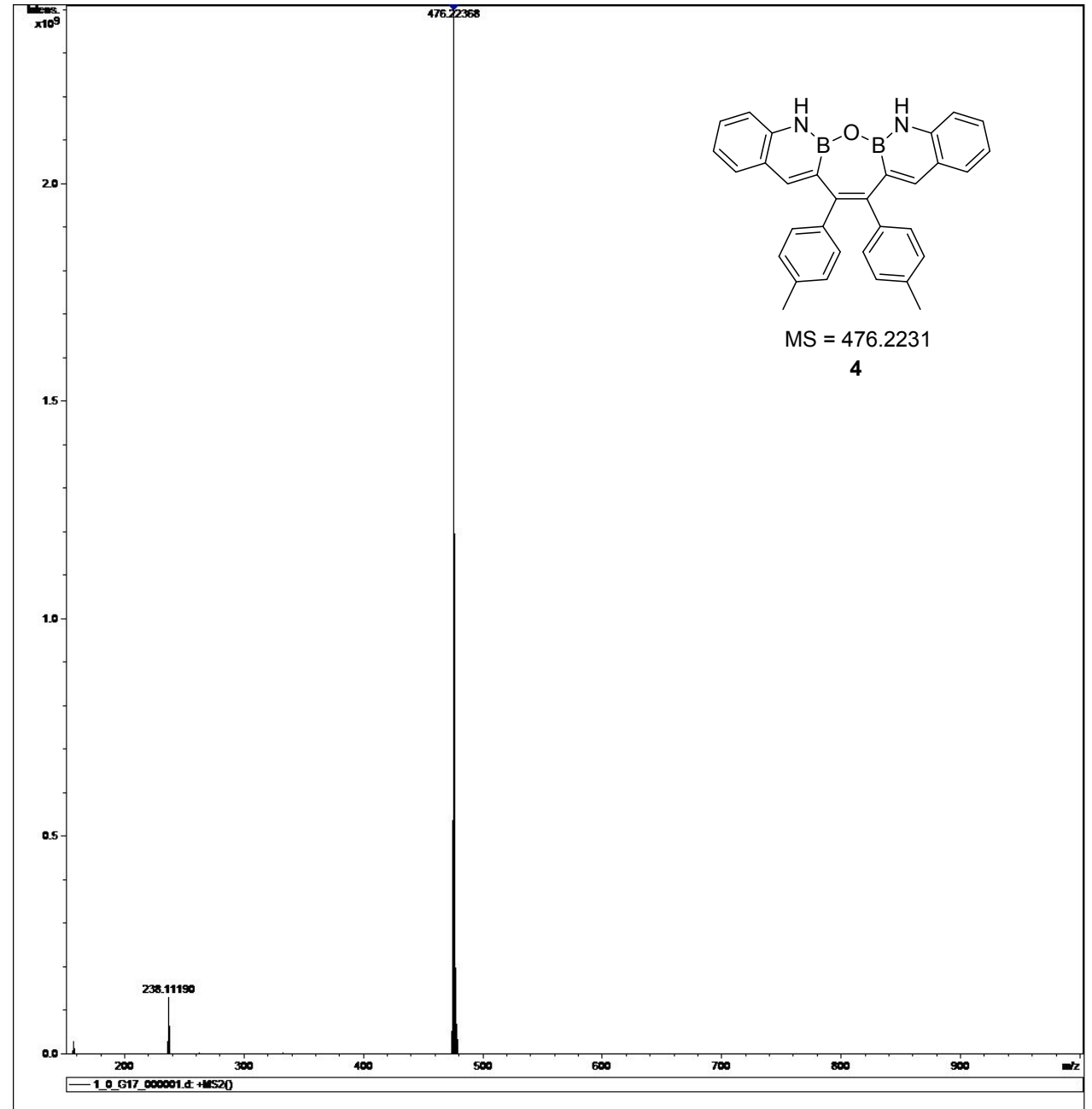

${ }^{1} \mathrm{H}$ - NMR spectrum of 5

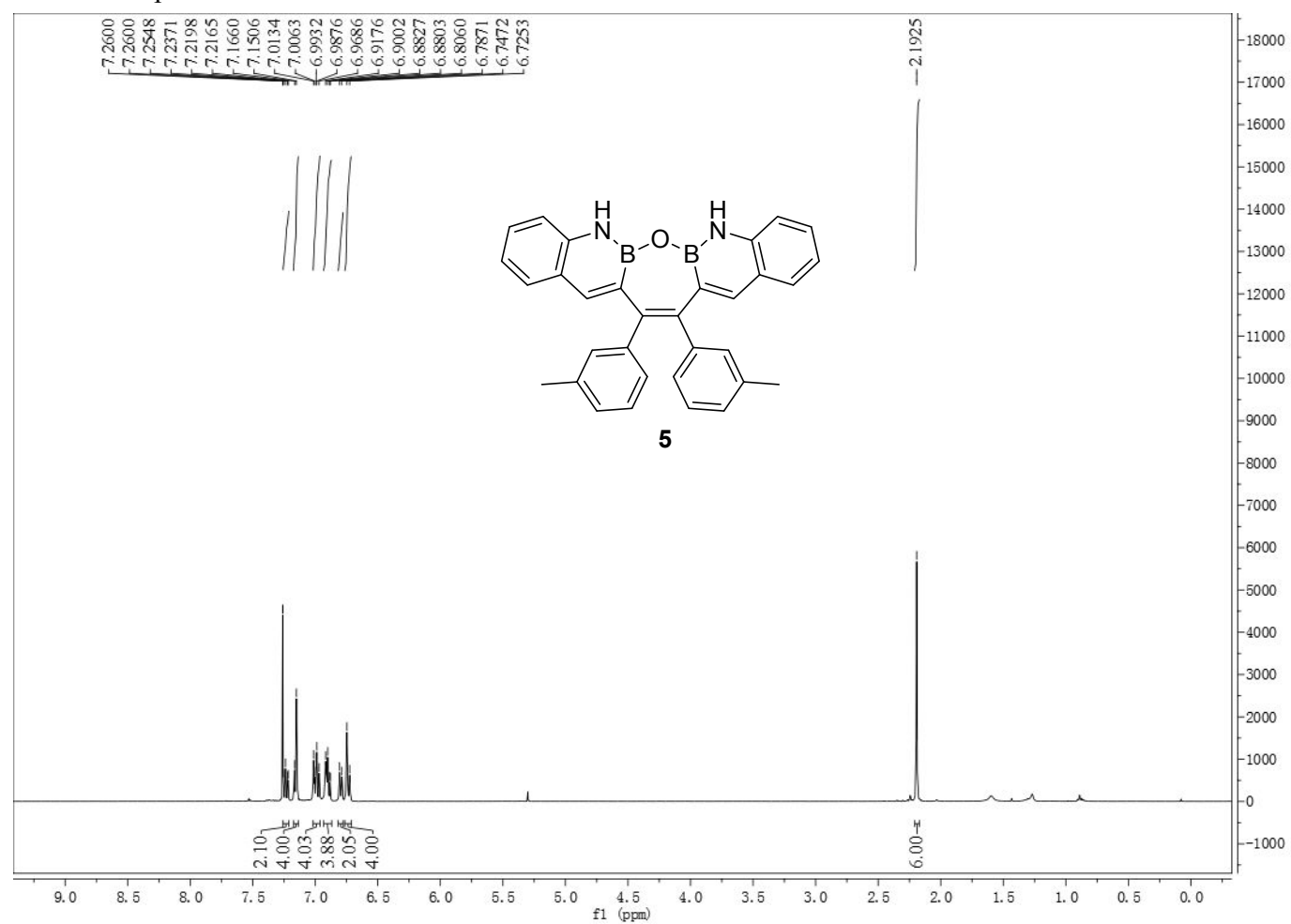

${ }^{13} \mathrm{C}$ - NMR spectrum of 5 


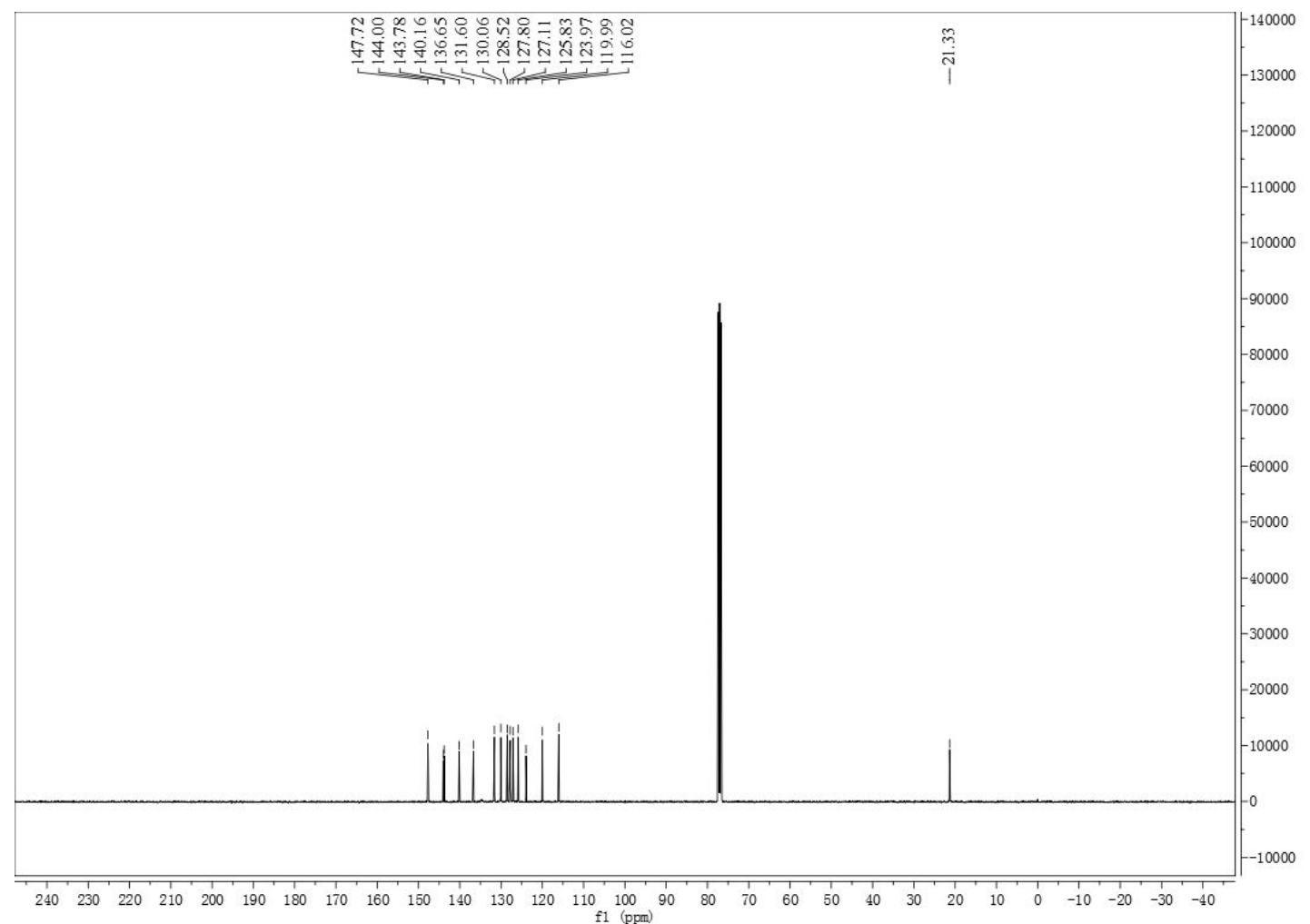

${ }^{13} \mathrm{~B}$ - NMR spectrum of $\mathbf{5}$

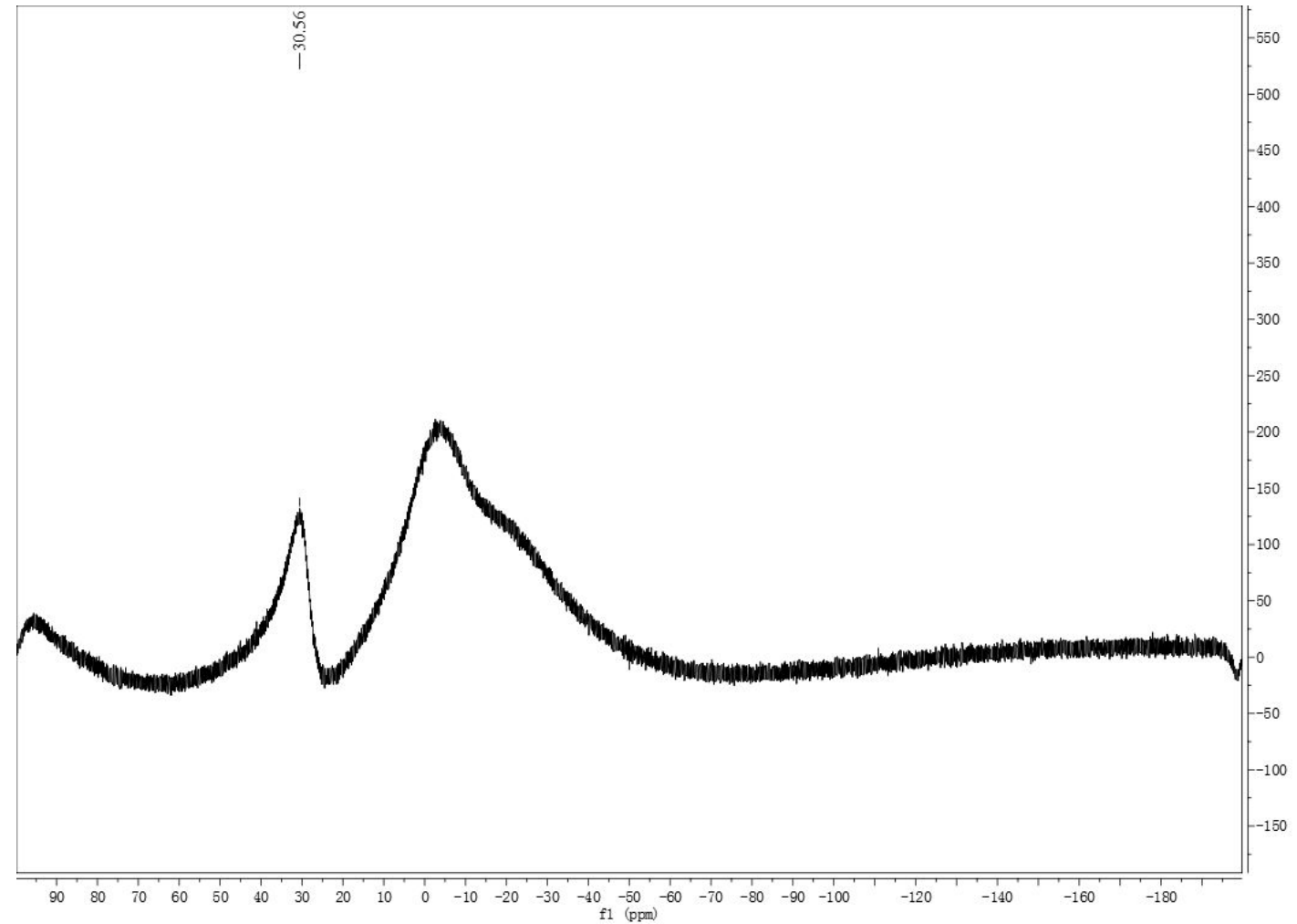

HRMS spectrum of $\mathbf{5}$ 


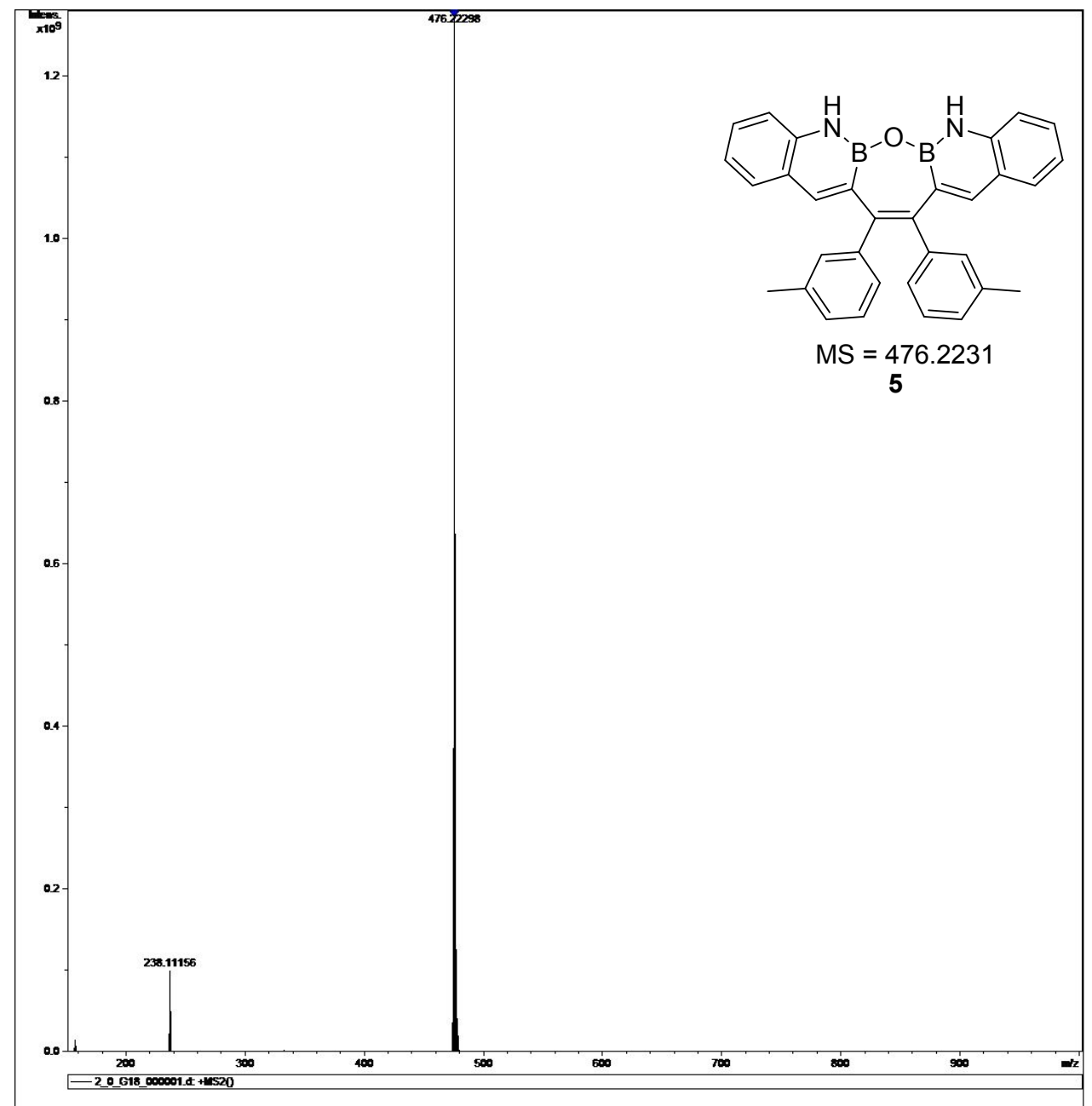

${ }^{1} \mathrm{H}$ - NMR spectrum of 6

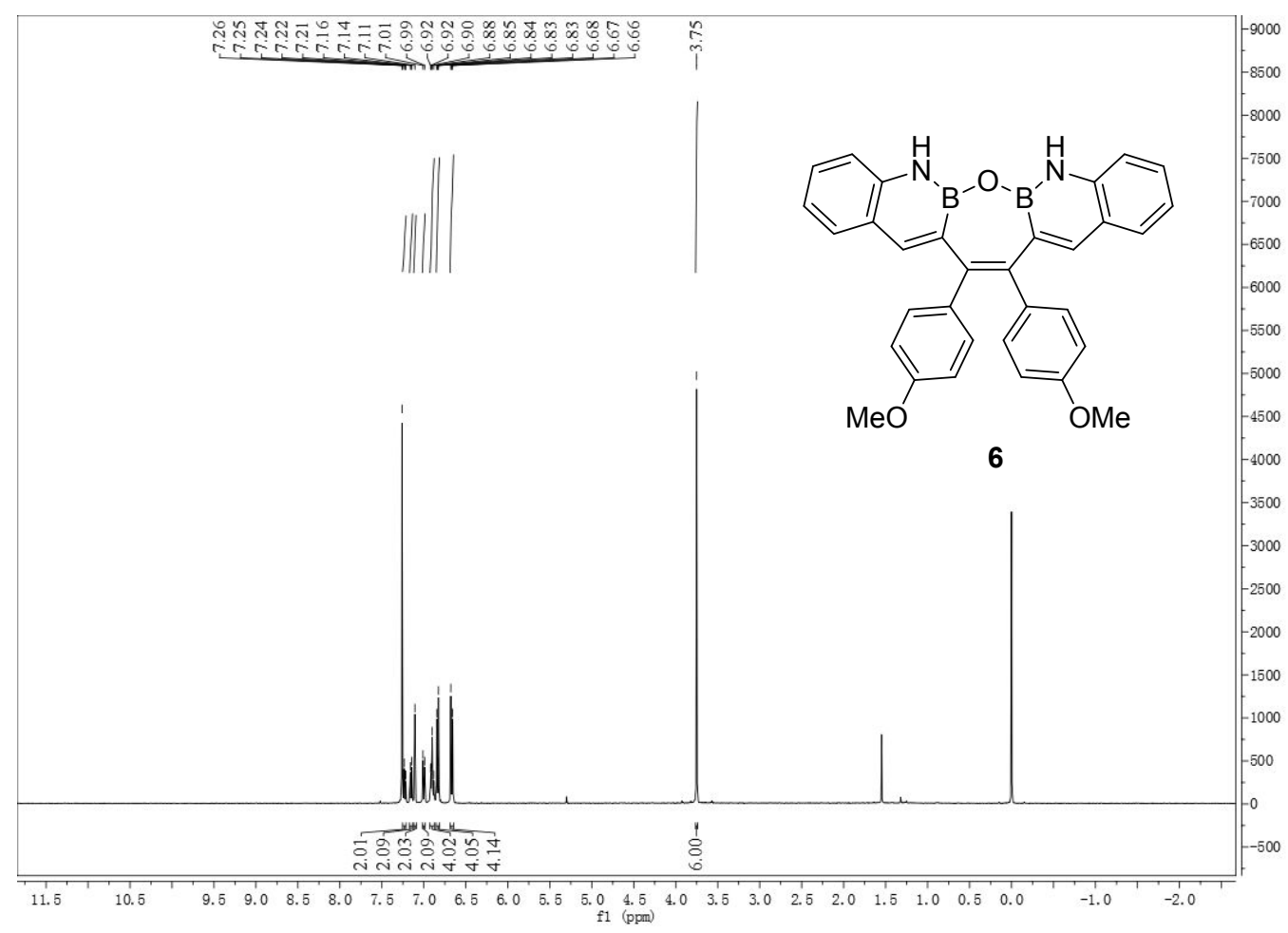

${ }^{13} \mathrm{C}$ - NMR spectrum of 6 


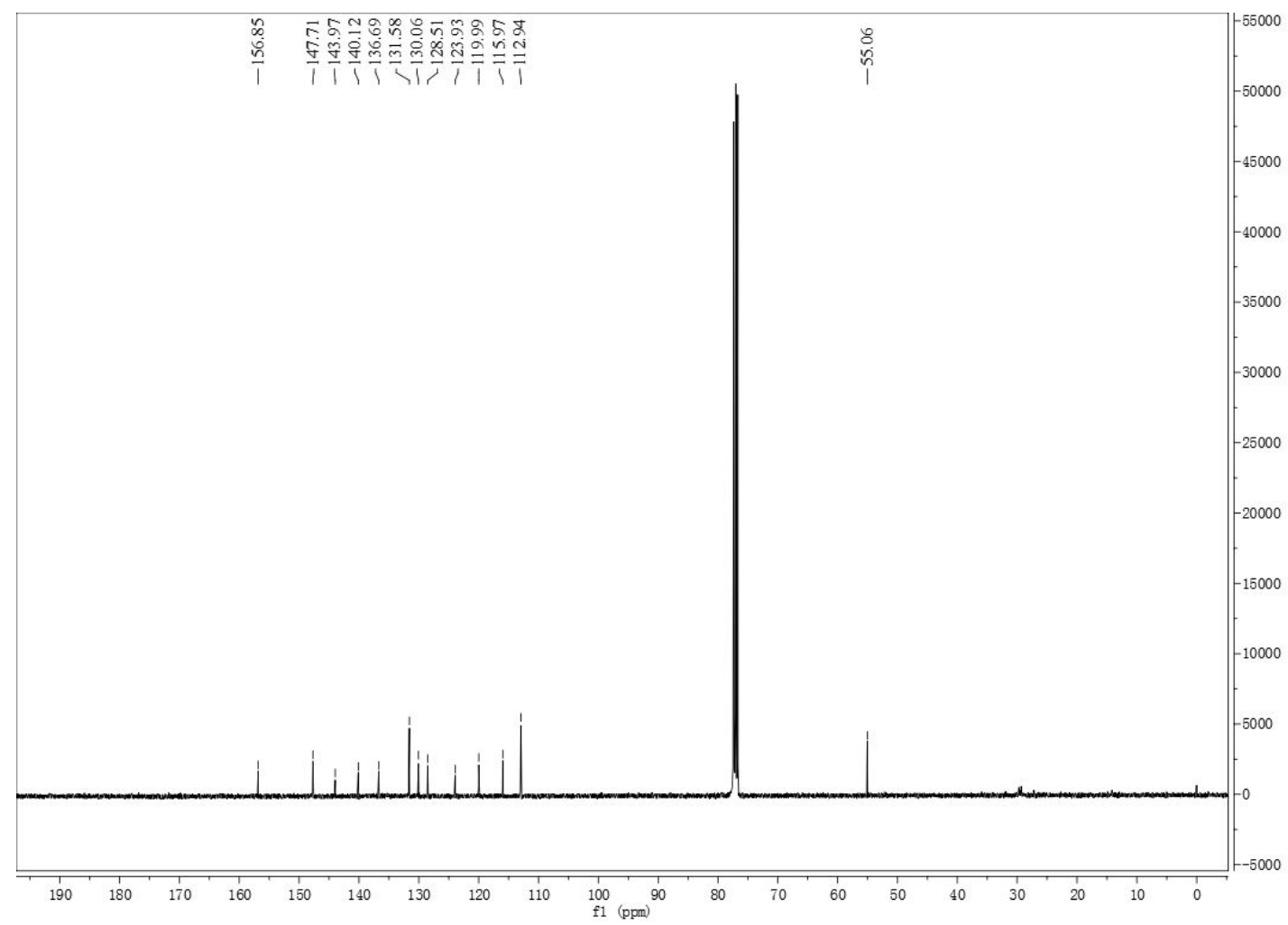

${ }^{11} \mathrm{~B}$ - NMR spectrum of 6

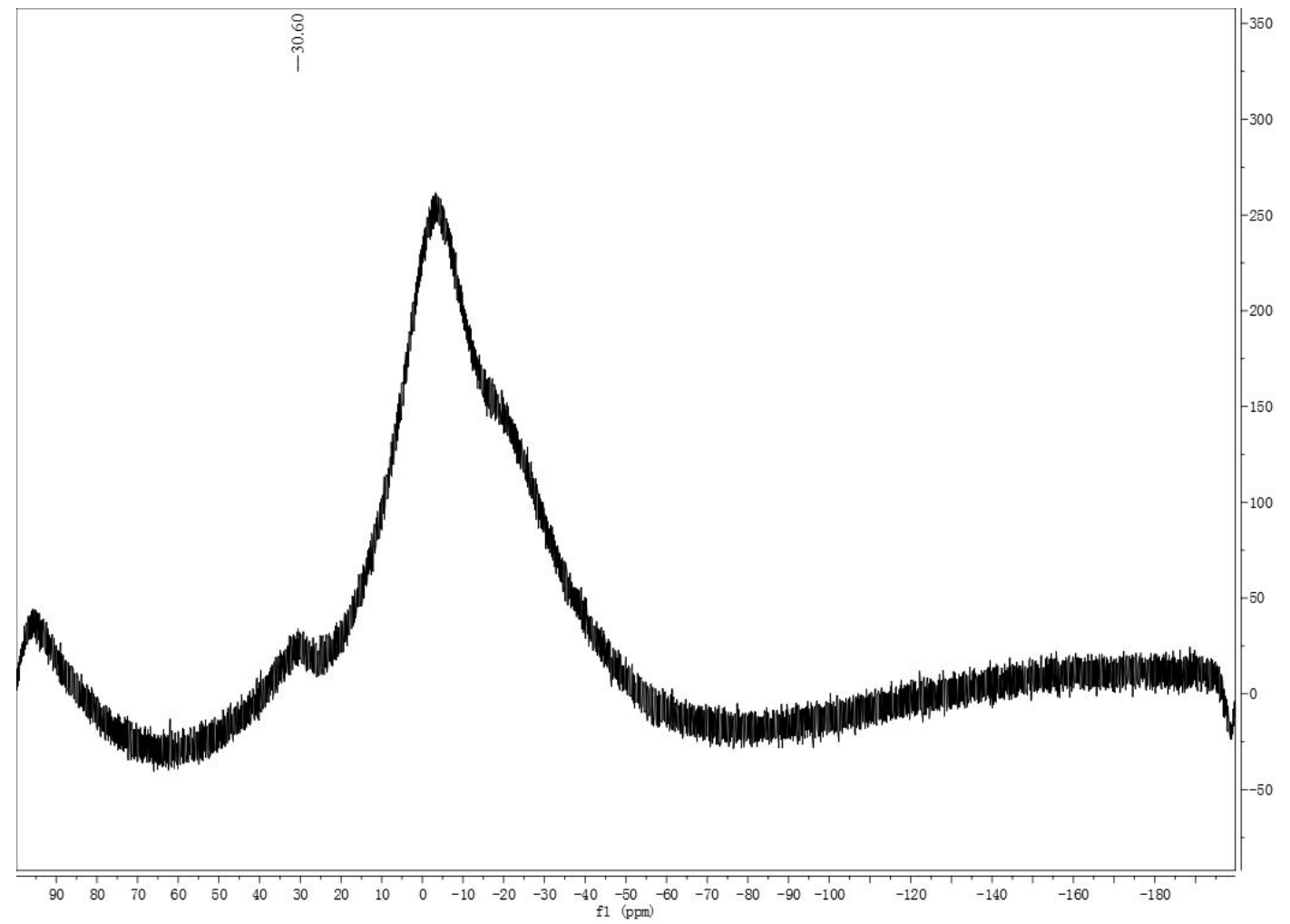

HRMS spectrum of 6 


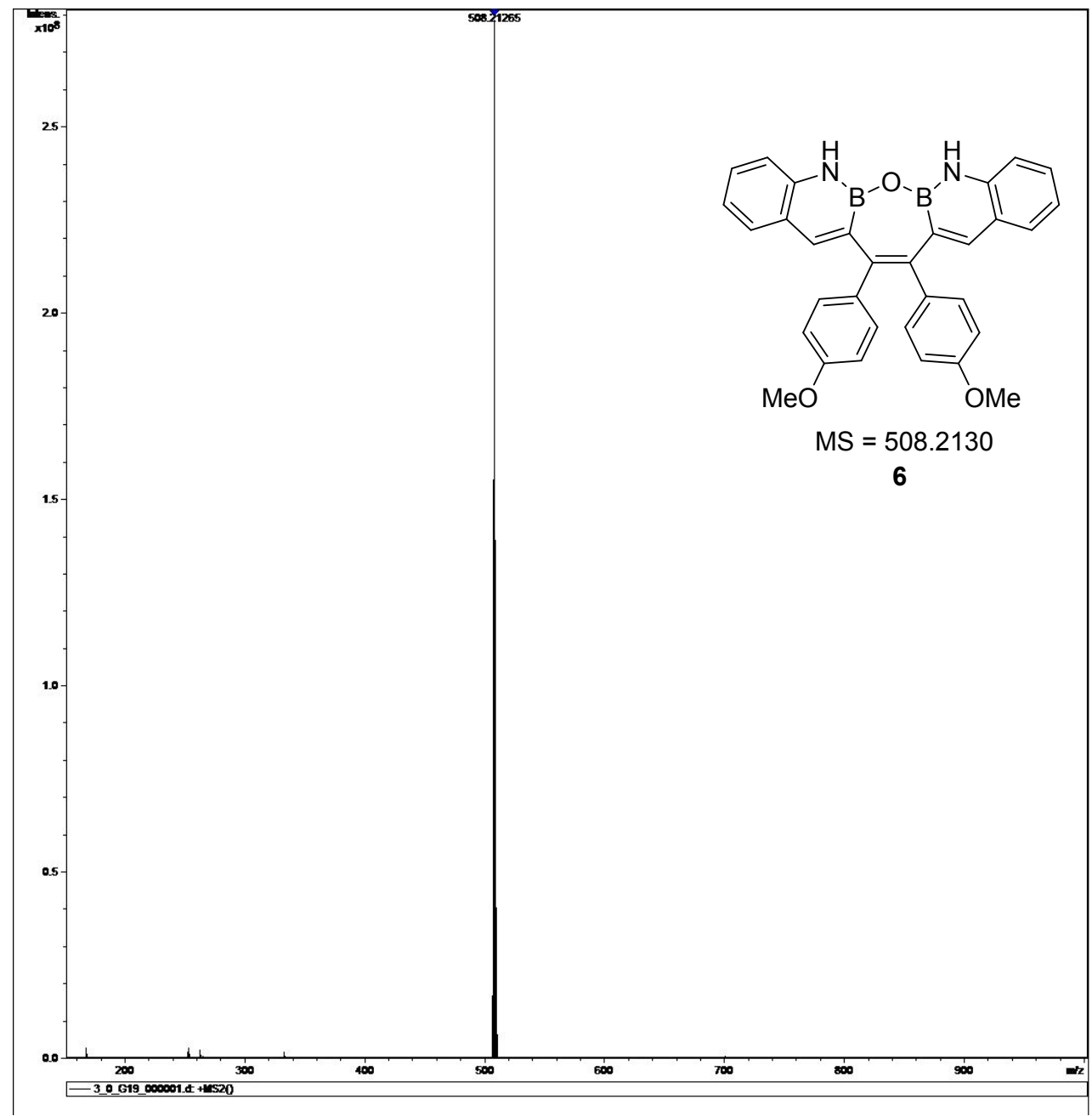

${ }^{1} \mathrm{H}$ - NMR spectrum of 7

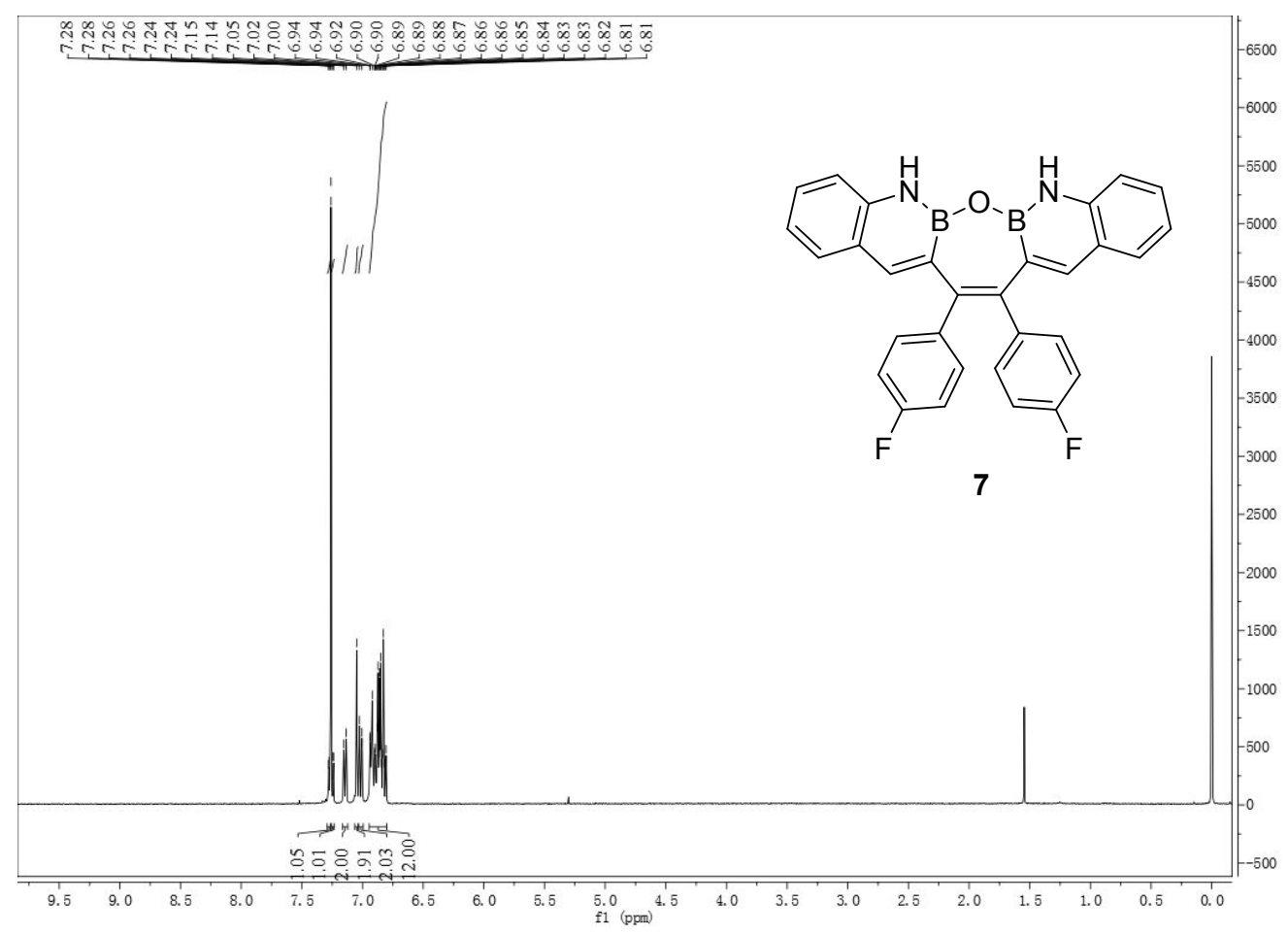


${ }^{13} \mathrm{C}$ - NMR spectrum of 7

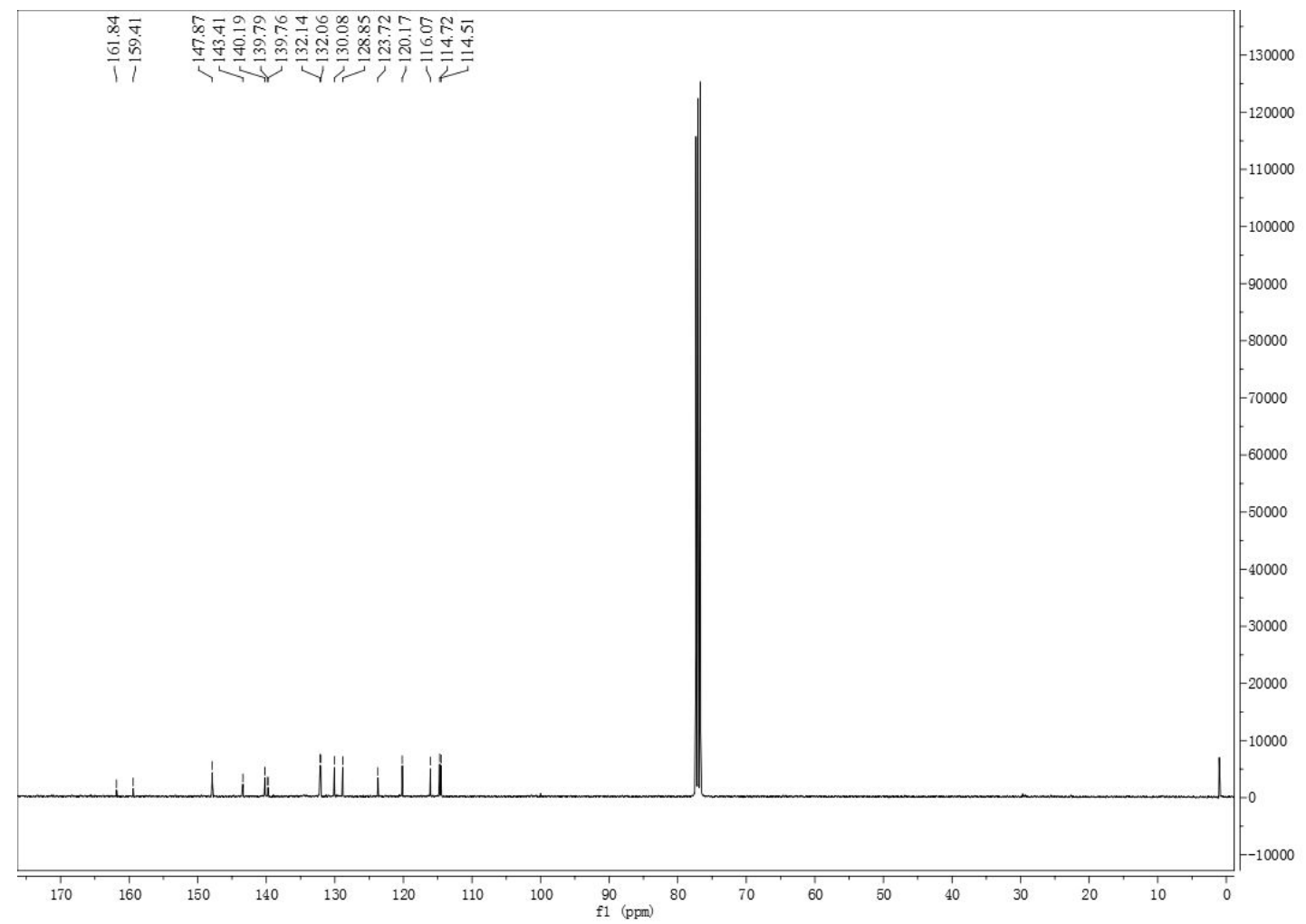

${ }^{11} \mathrm{~B}$ - NMR spectrum of 7

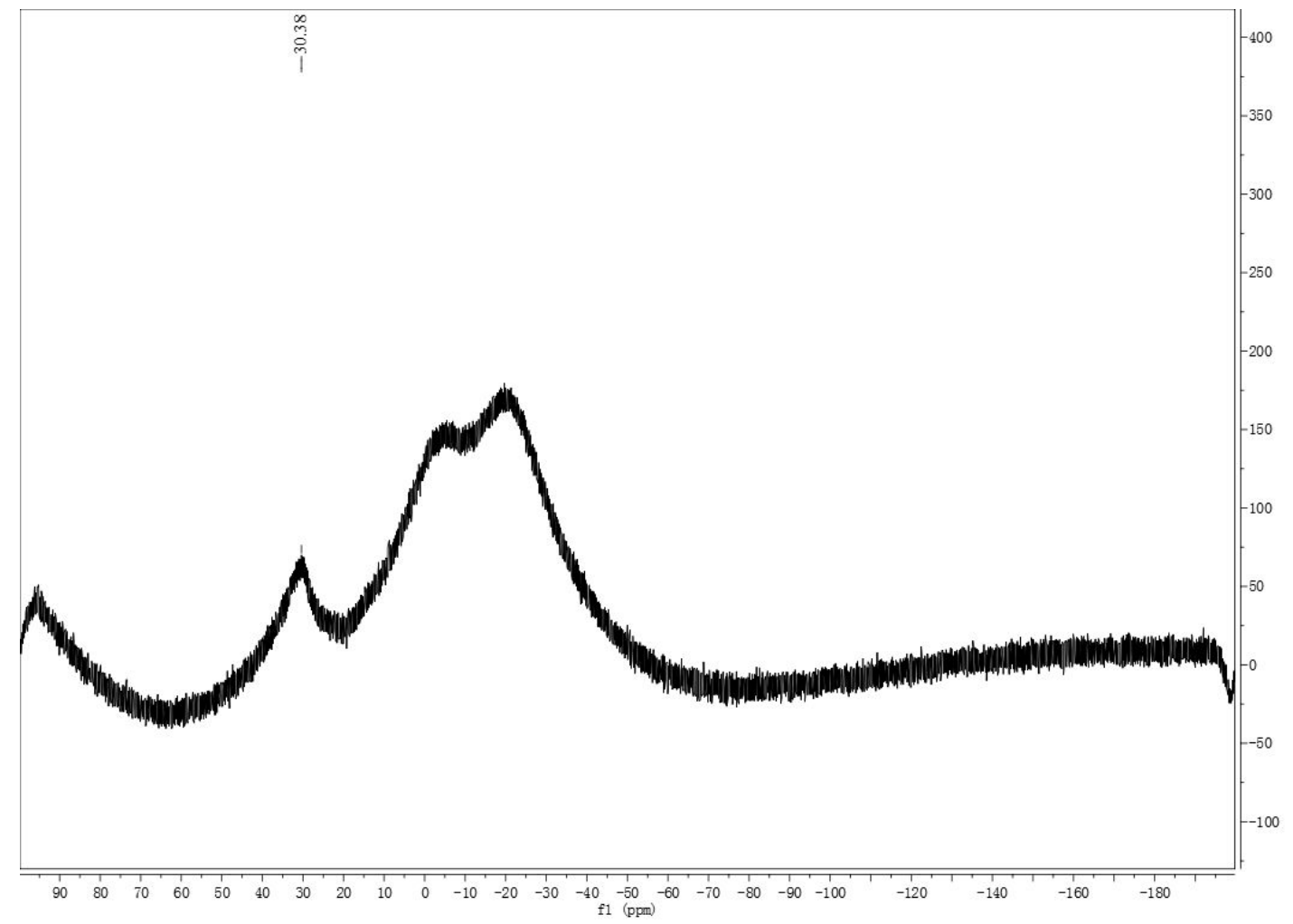


${ }^{19} \mathrm{~F}$ - NMR spectrum of 7

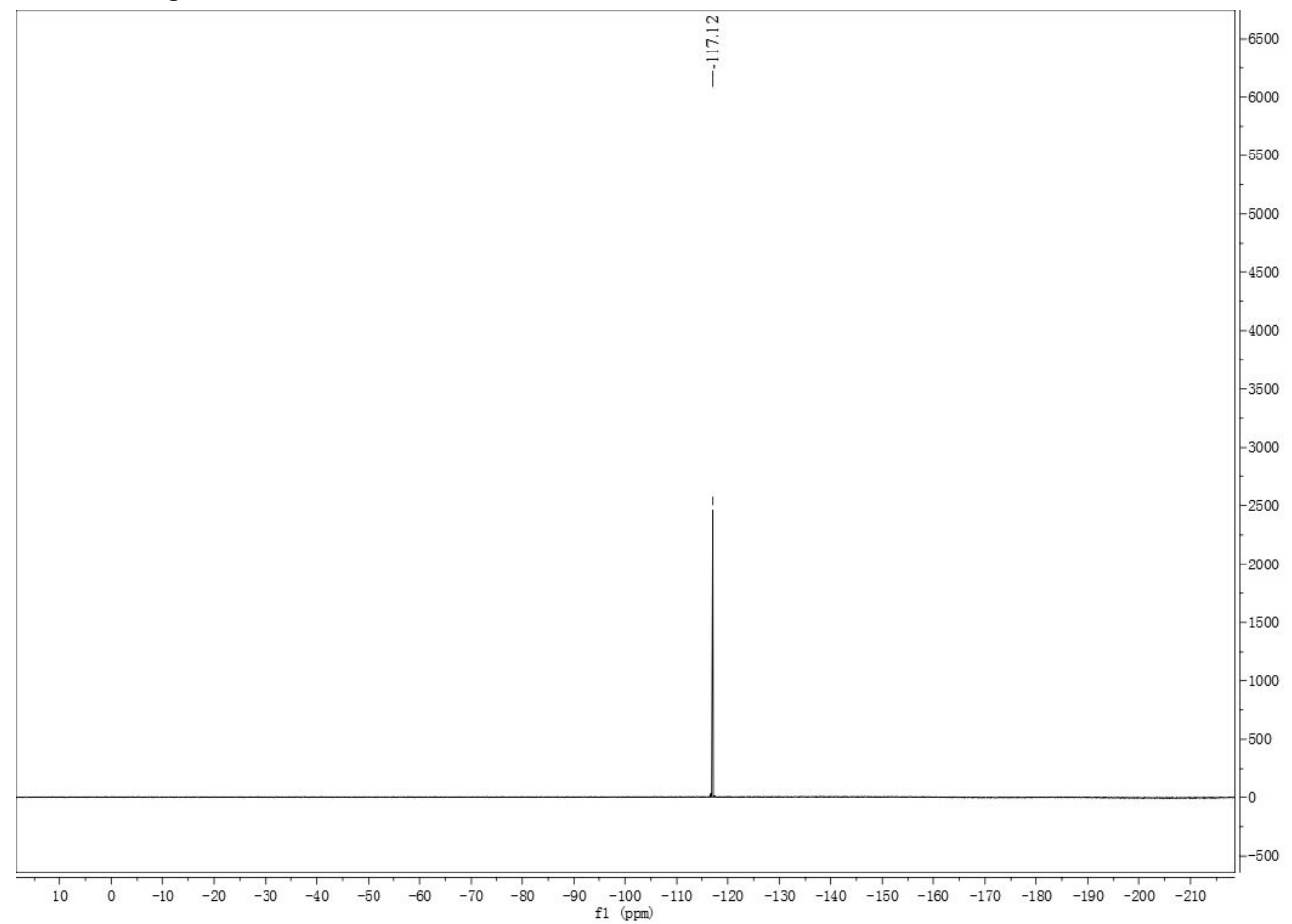

HRMS spectrum of 7

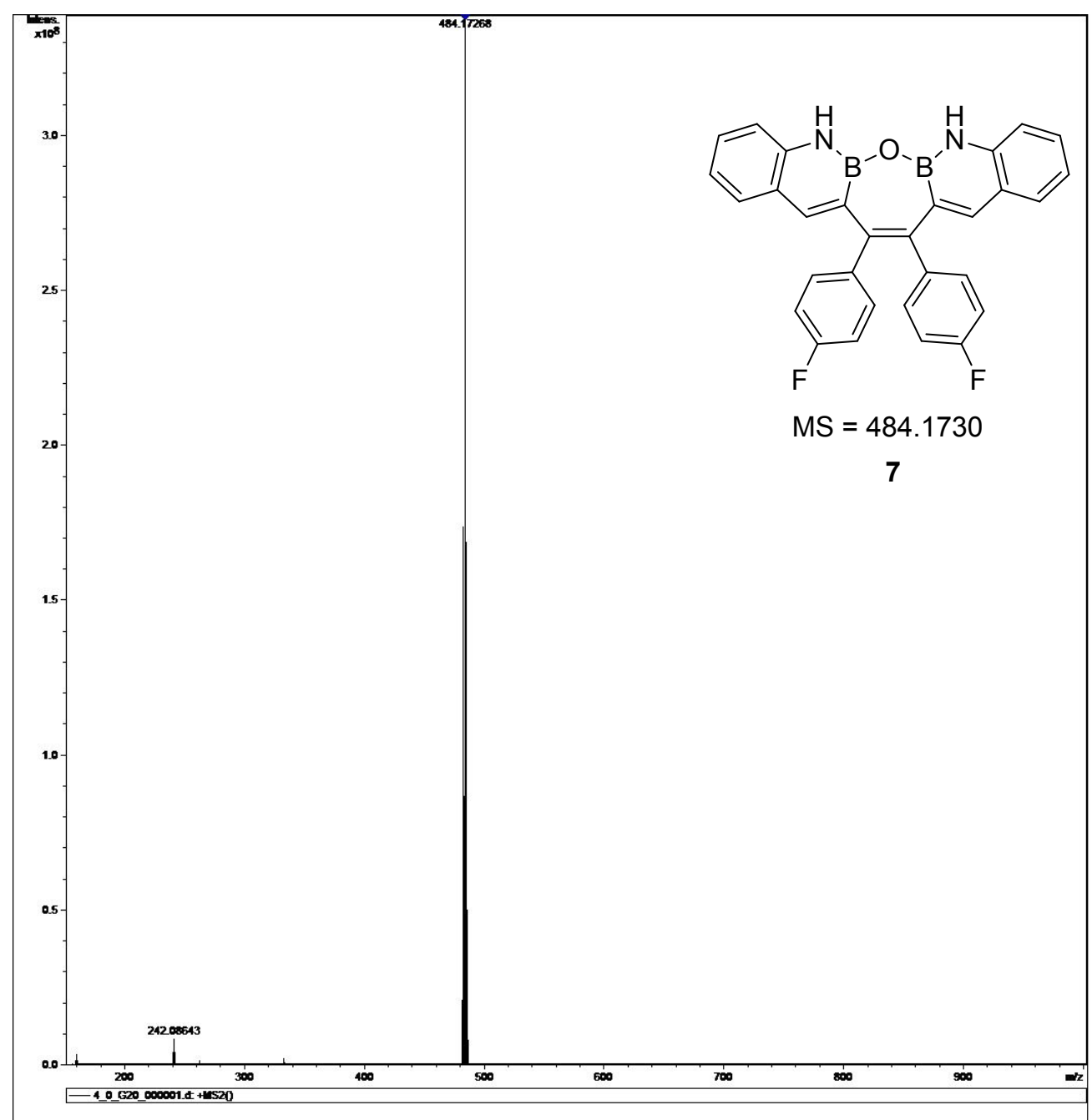


${ }^{1} \mathrm{H}$ - NMR spectrum of 8

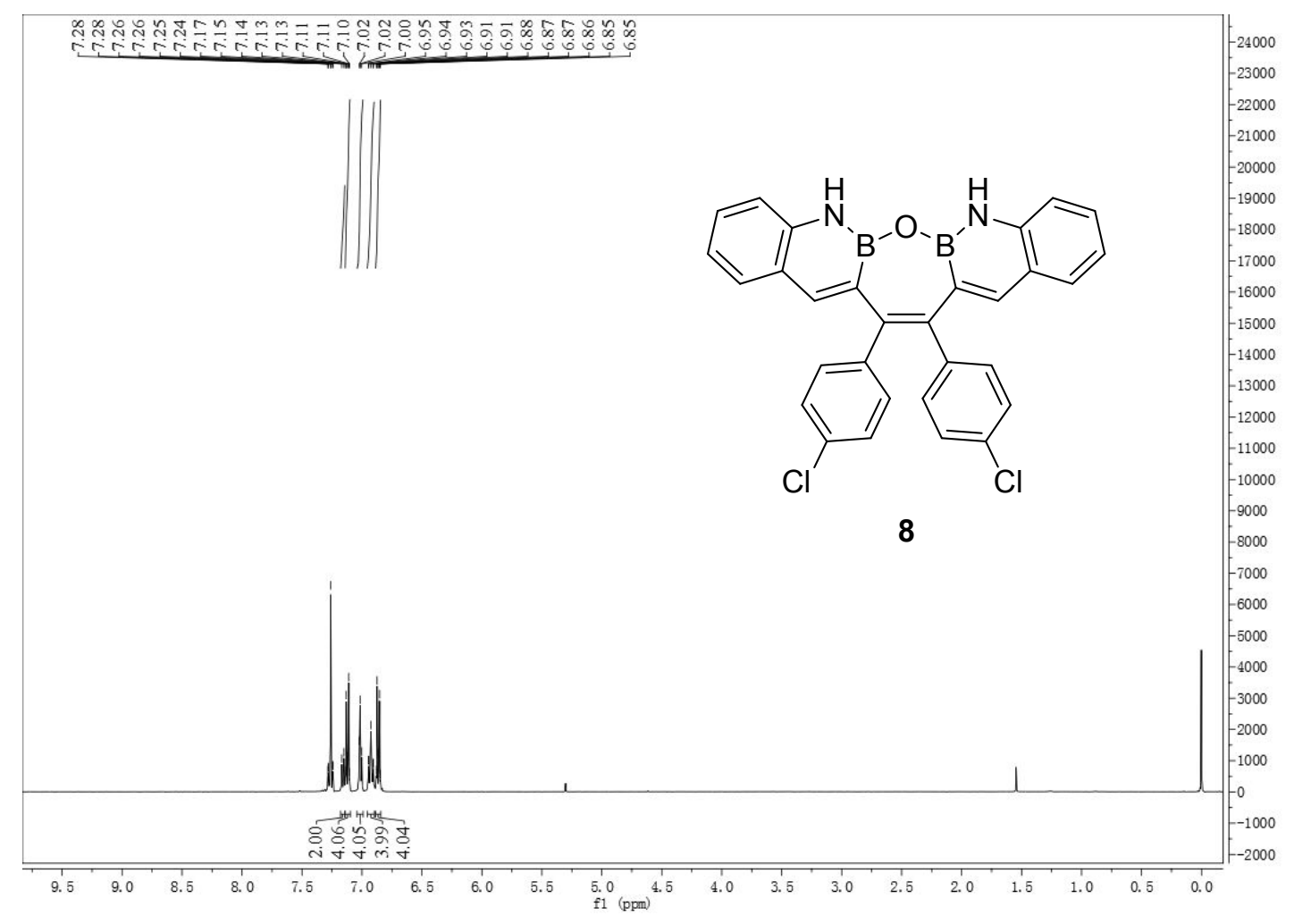

${ }^{13} \mathrm{C}$ - NMR spectrum of 8

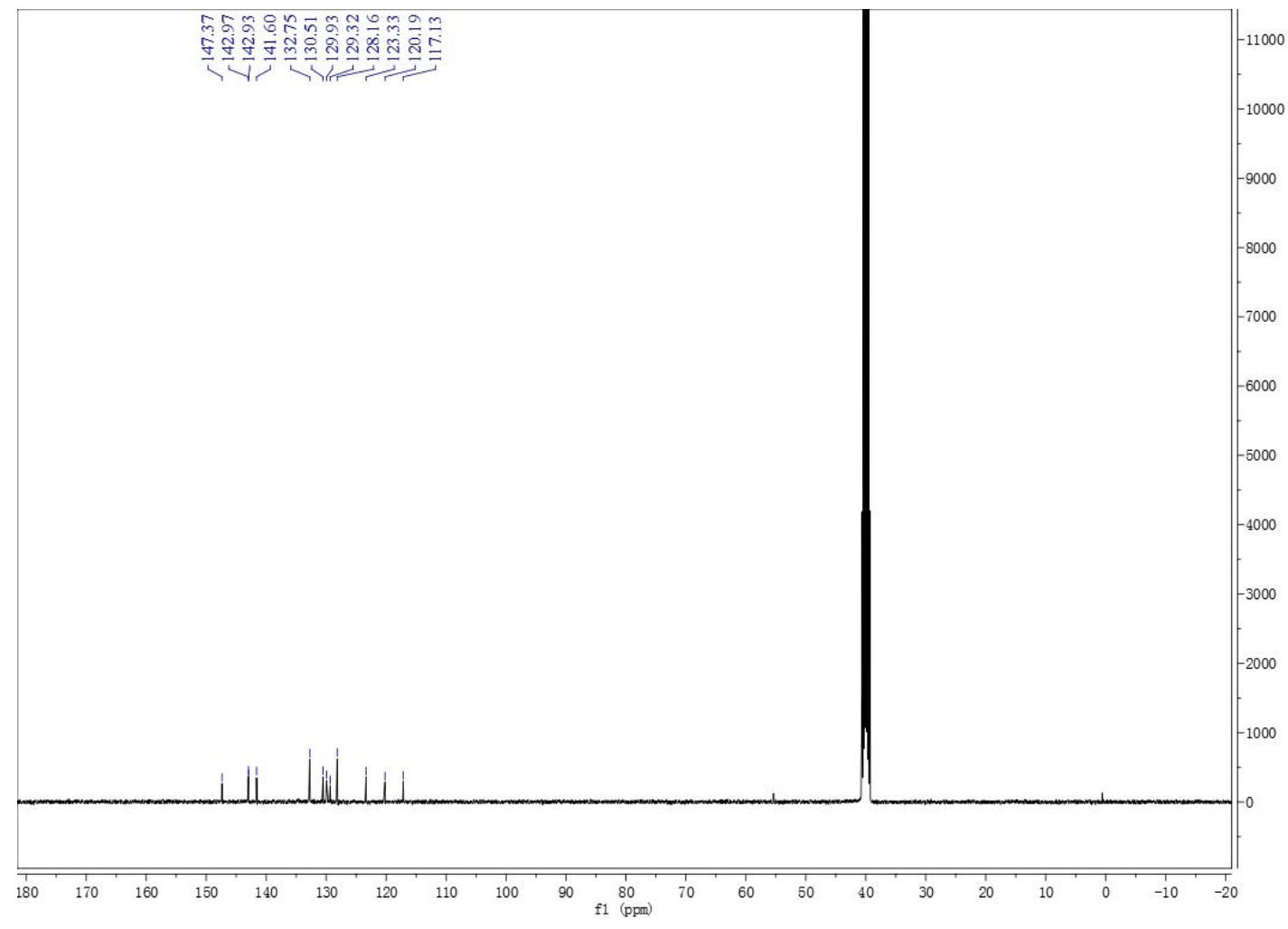


${ }^{11} \mathrm{~B}$ - NMR spectrum of 8

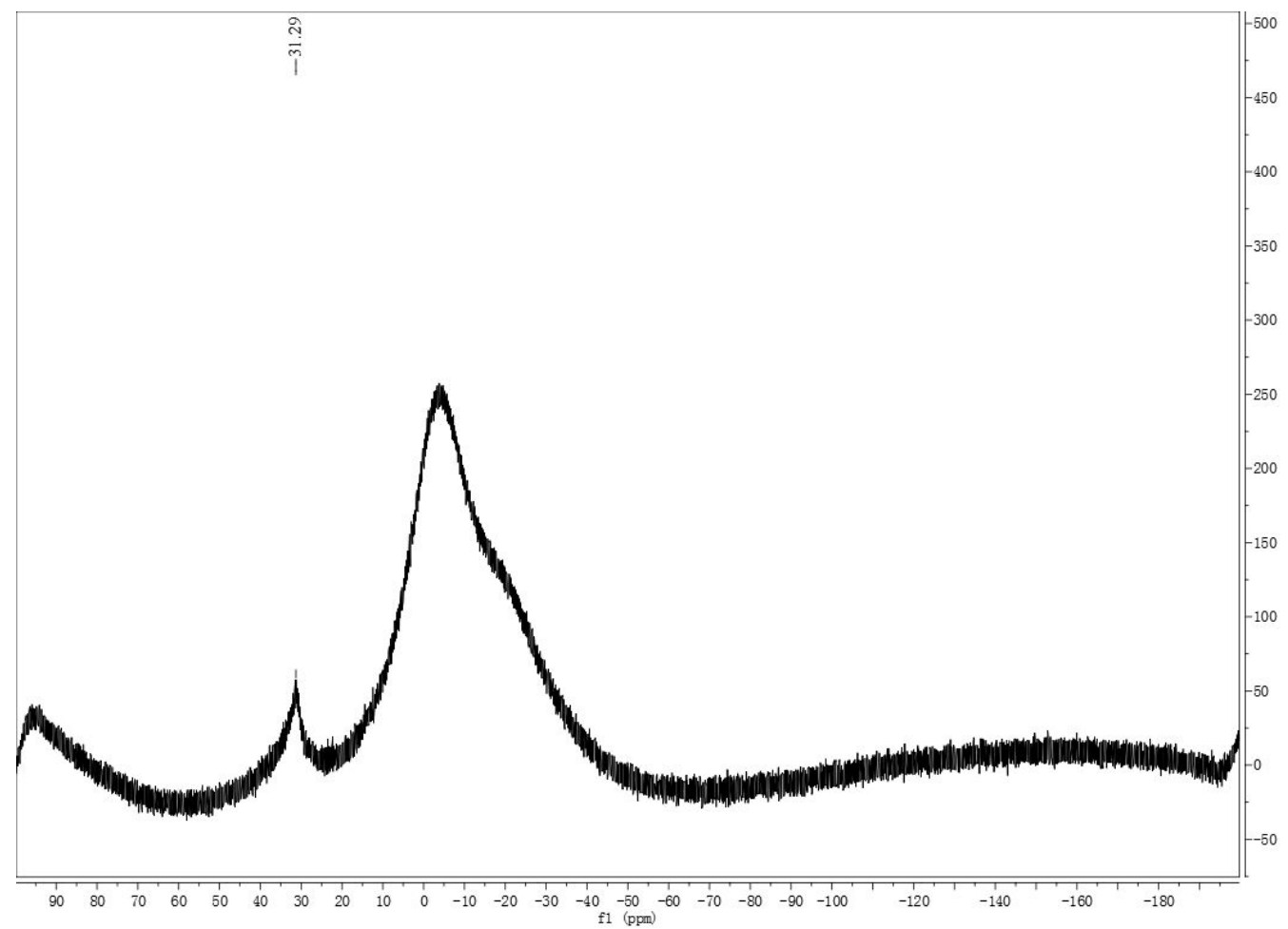

HRMS spectrum of 8

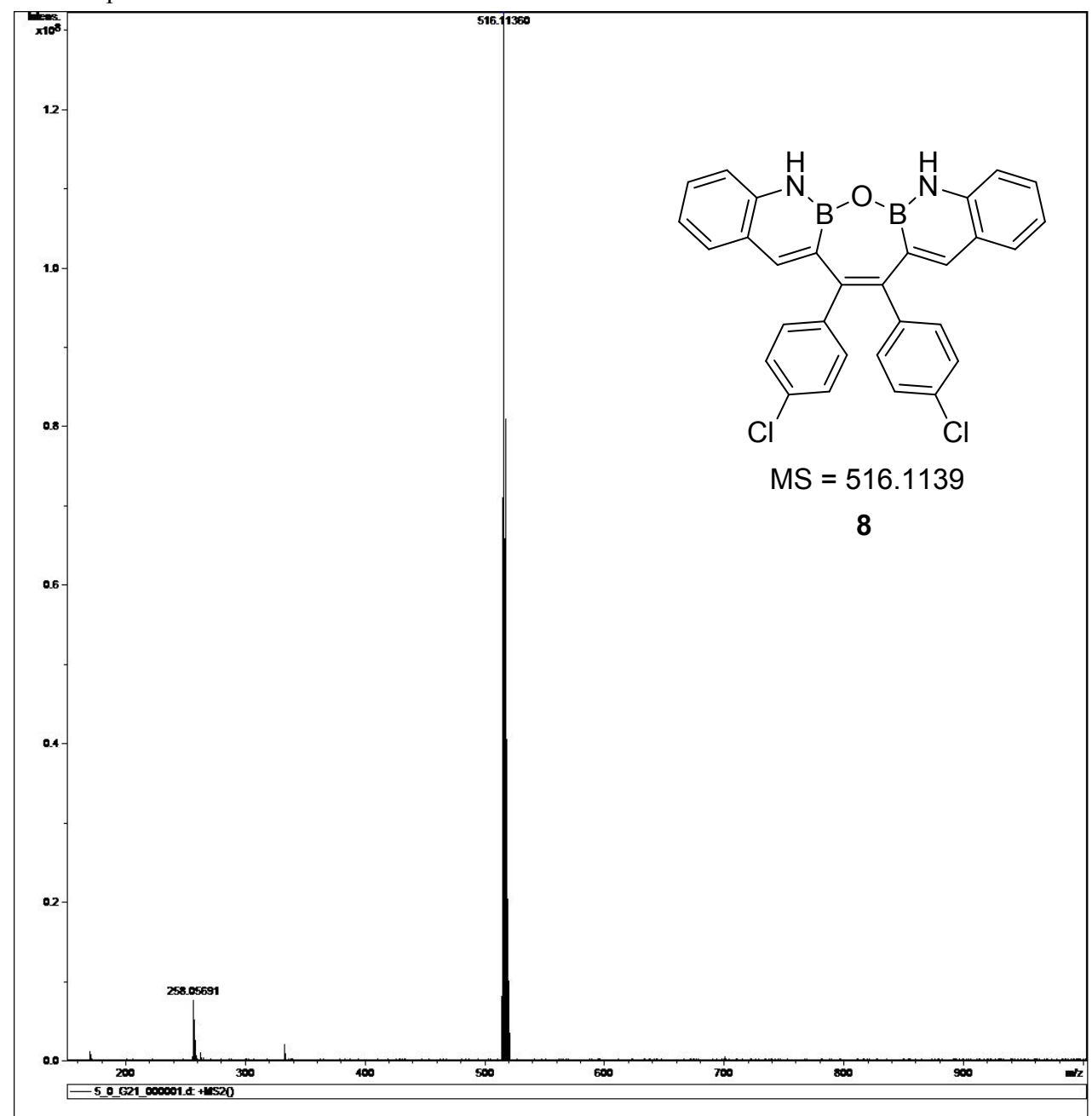


${ }^{1} \mathrm{H}$ - NMR spectrum of 9

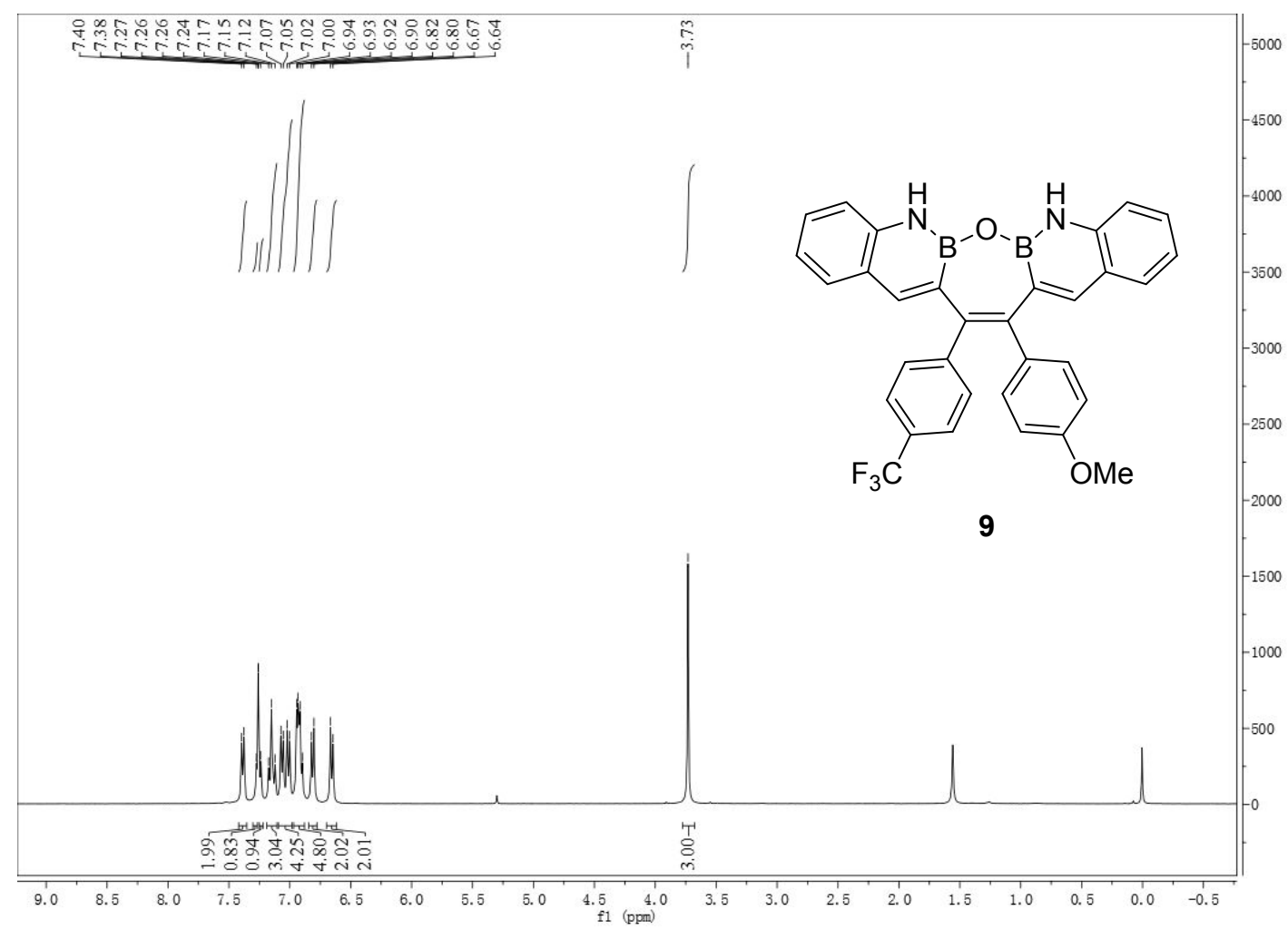

${ }^{13} \mathrm{C}$ - NMR spectrum of 9

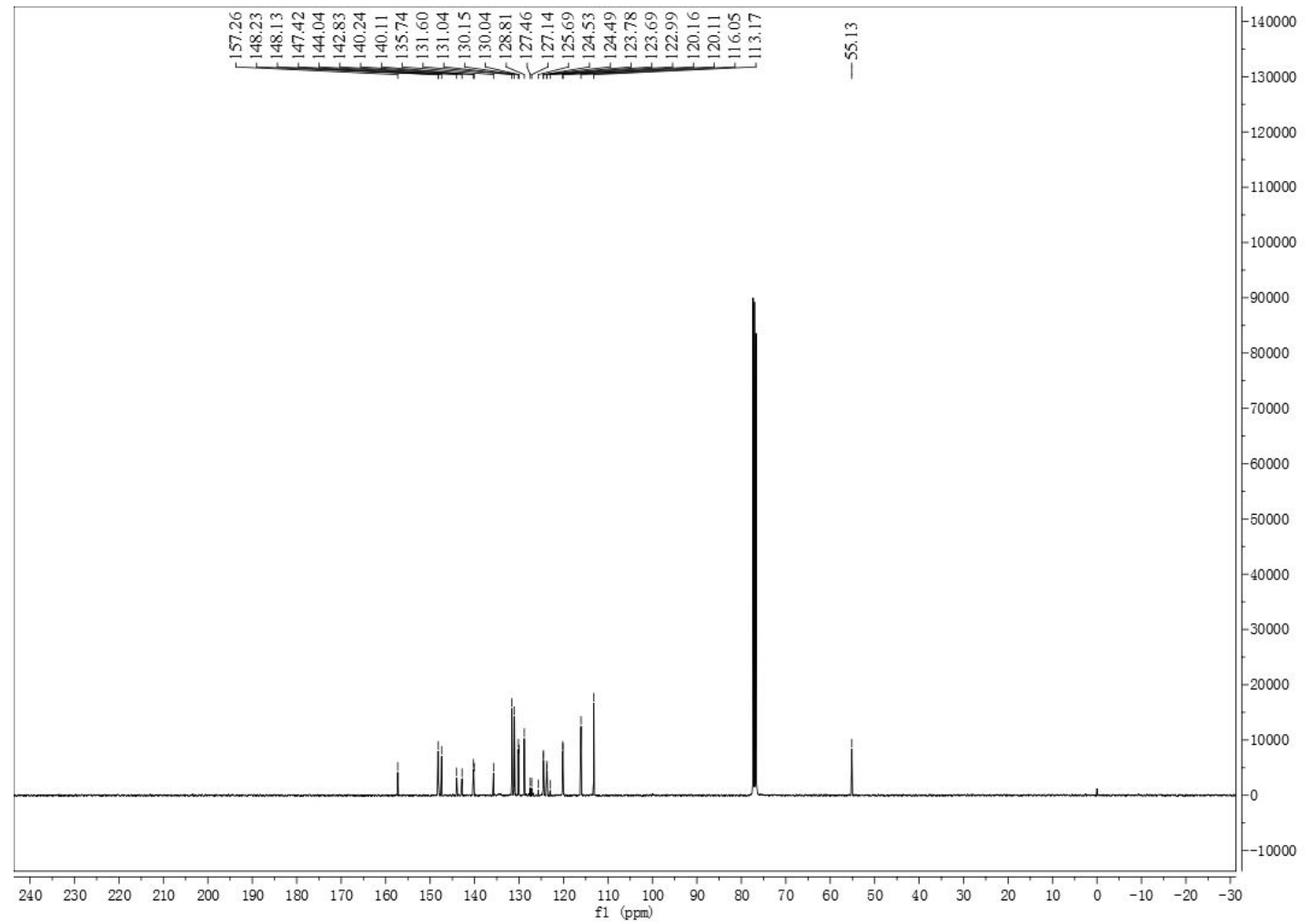


${ }^{11} \mathrm{~B}$ - NMR spectrum of 9

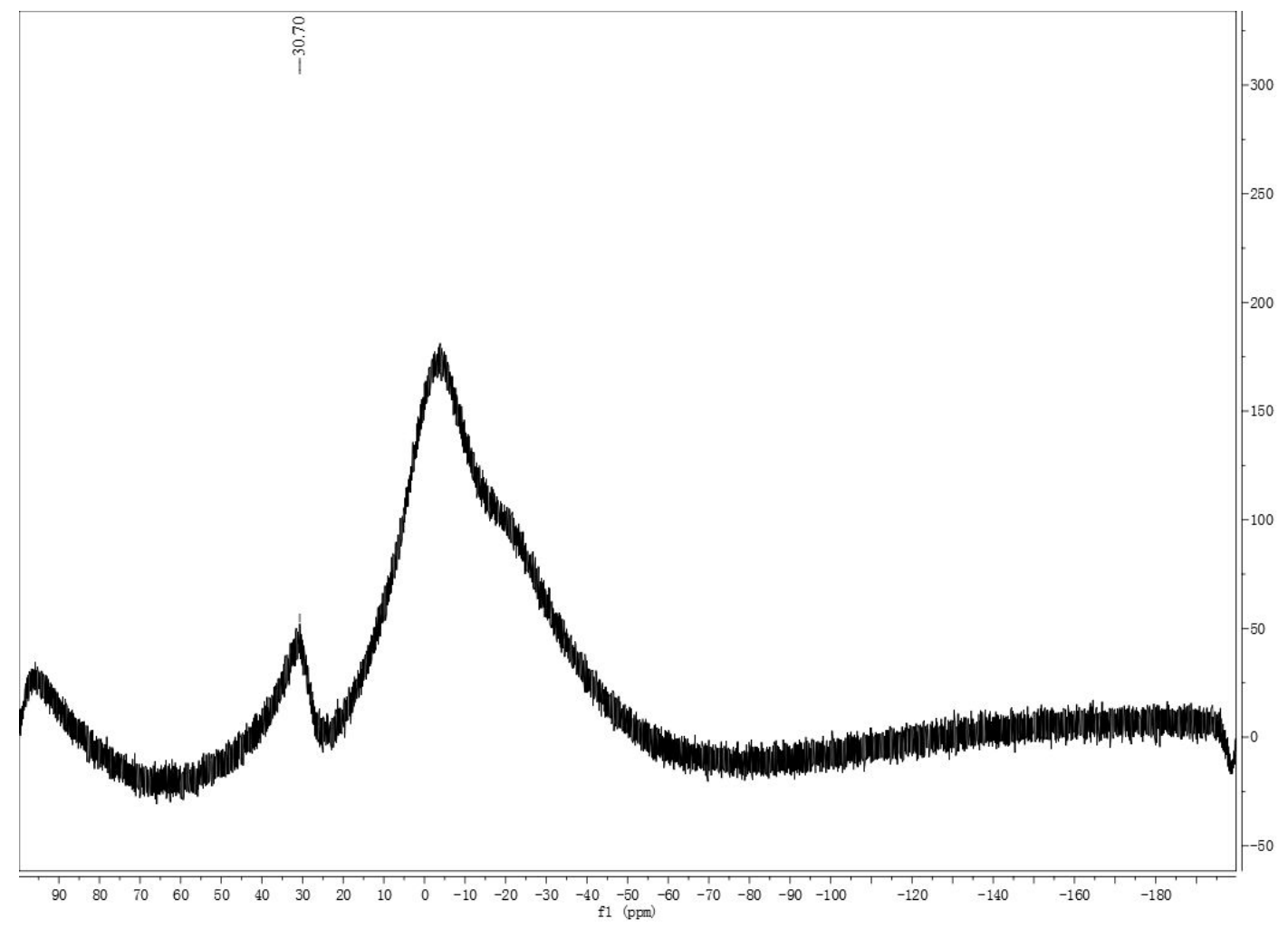

${ }^{19} \mathrm{~F}$ - NMR spectrum of 9

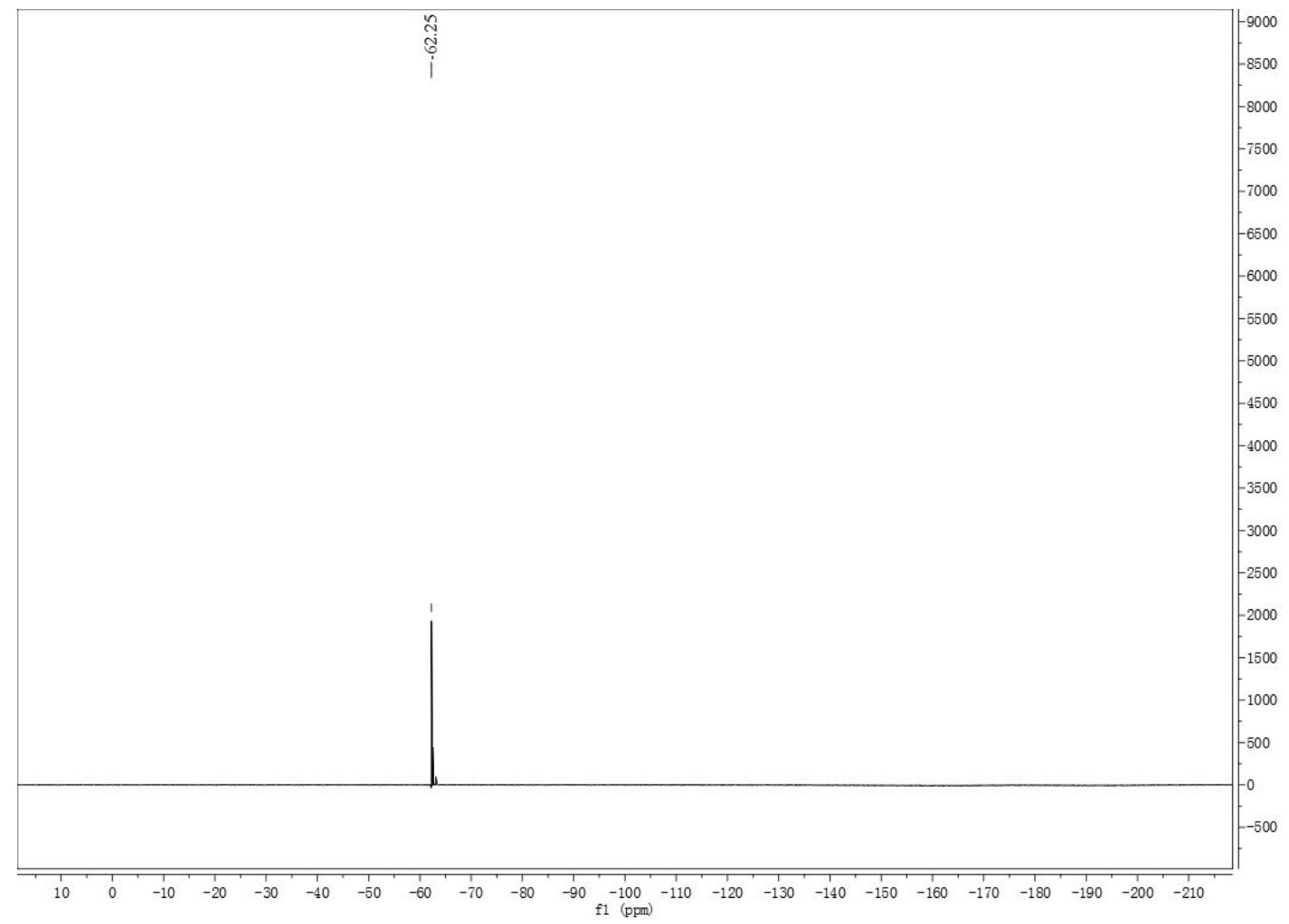


HRMS - NMR spectrum of 9

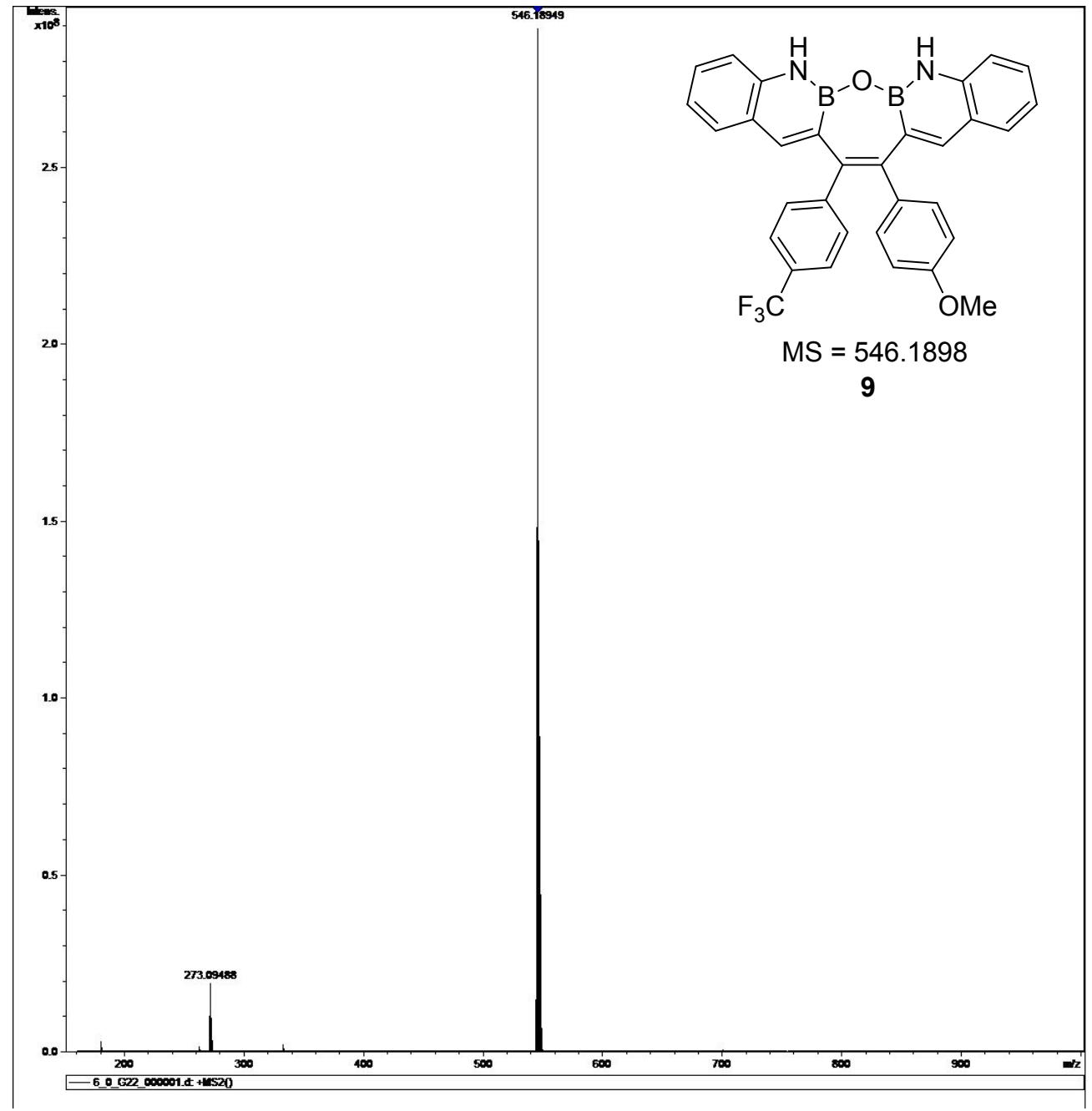

${ }^{1} \mathrm{H}$ - NMR spectrum of $\mathbf{1 0}$

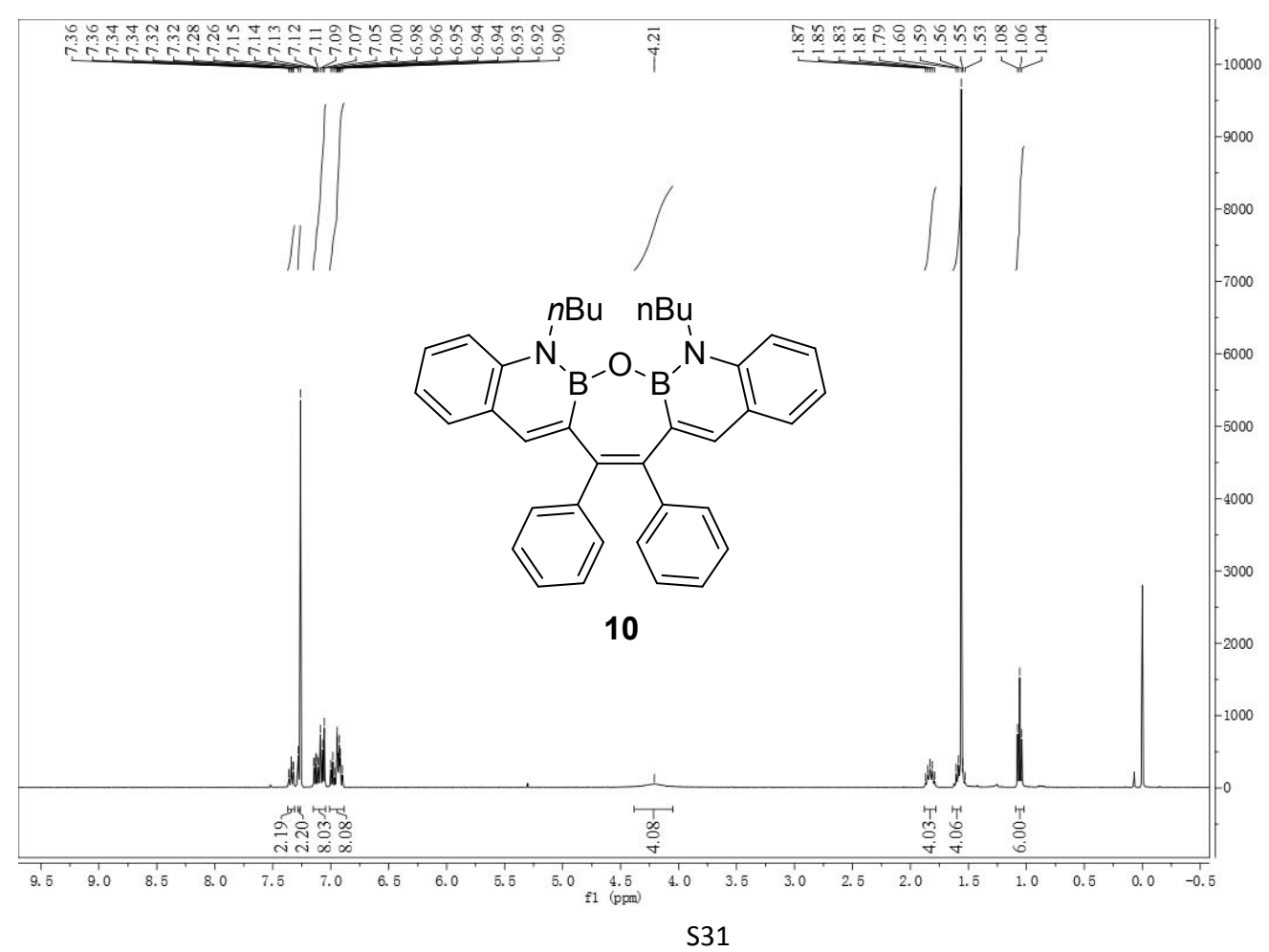


${ }^{11} \mathrm{C}$ - NMR spectrum of $\mathbf{1 0}$

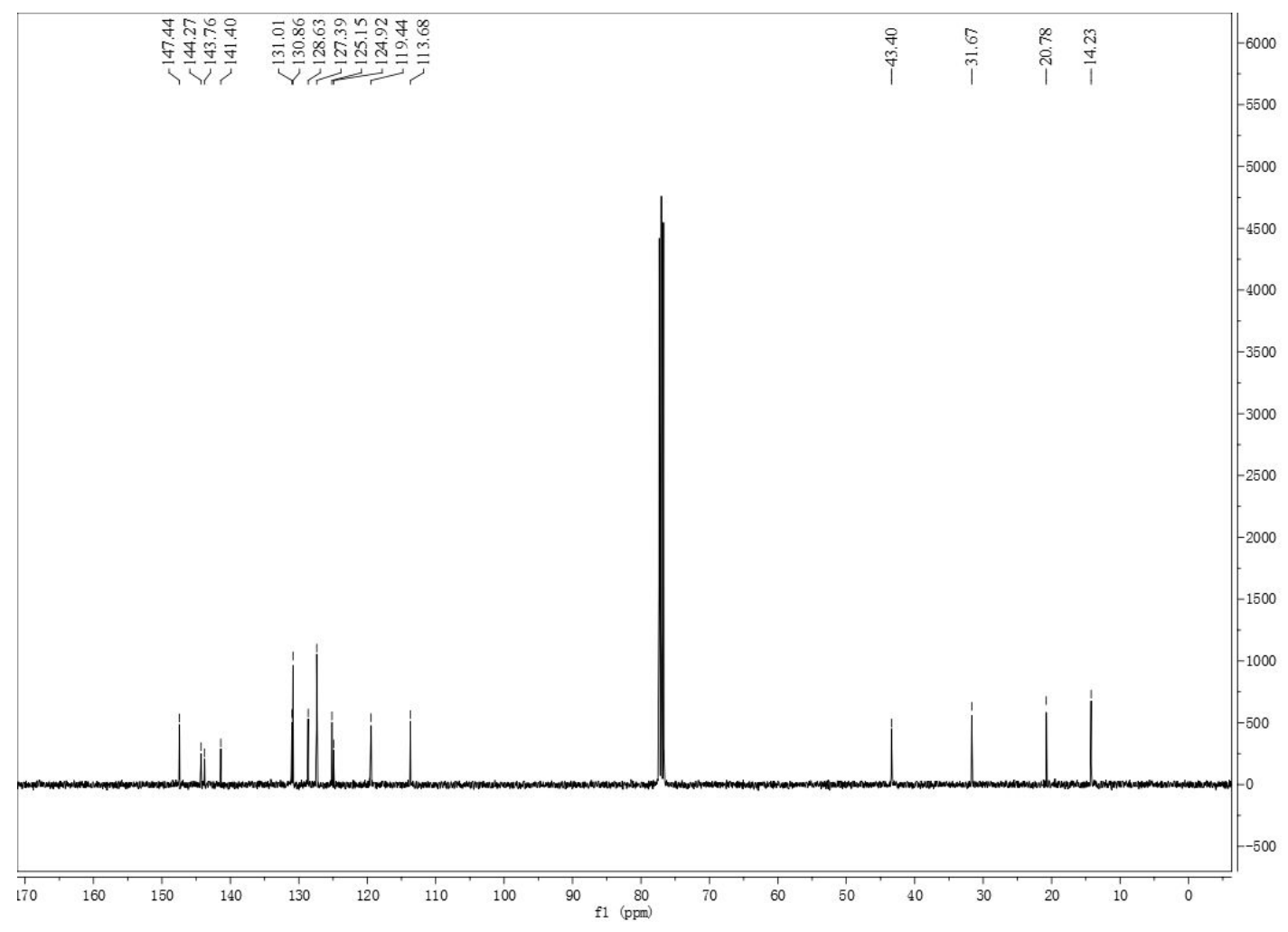

${ }^{11} \mathrm{~B}$ - NMR spectrum of $\mathbf{1 0}$

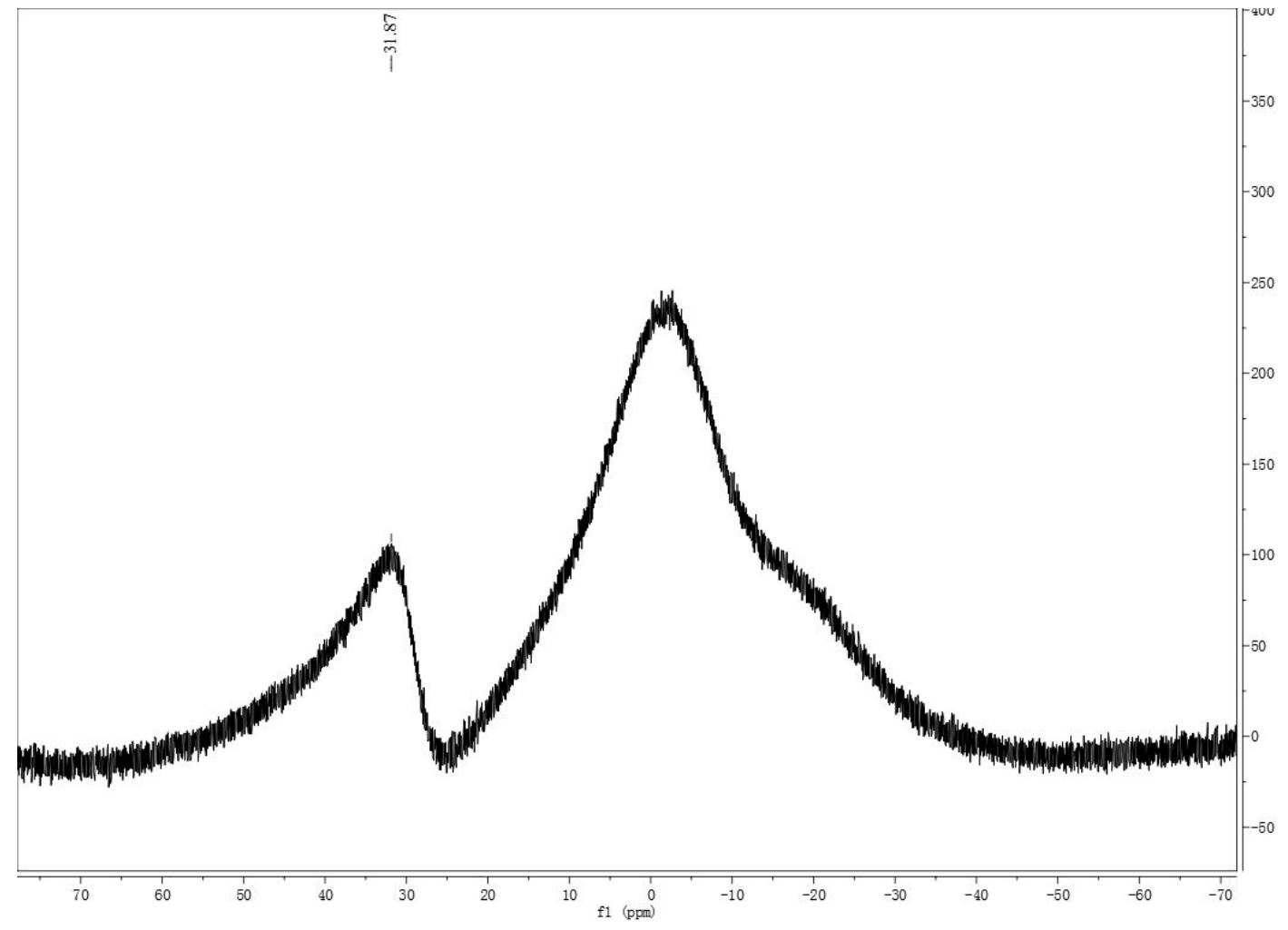




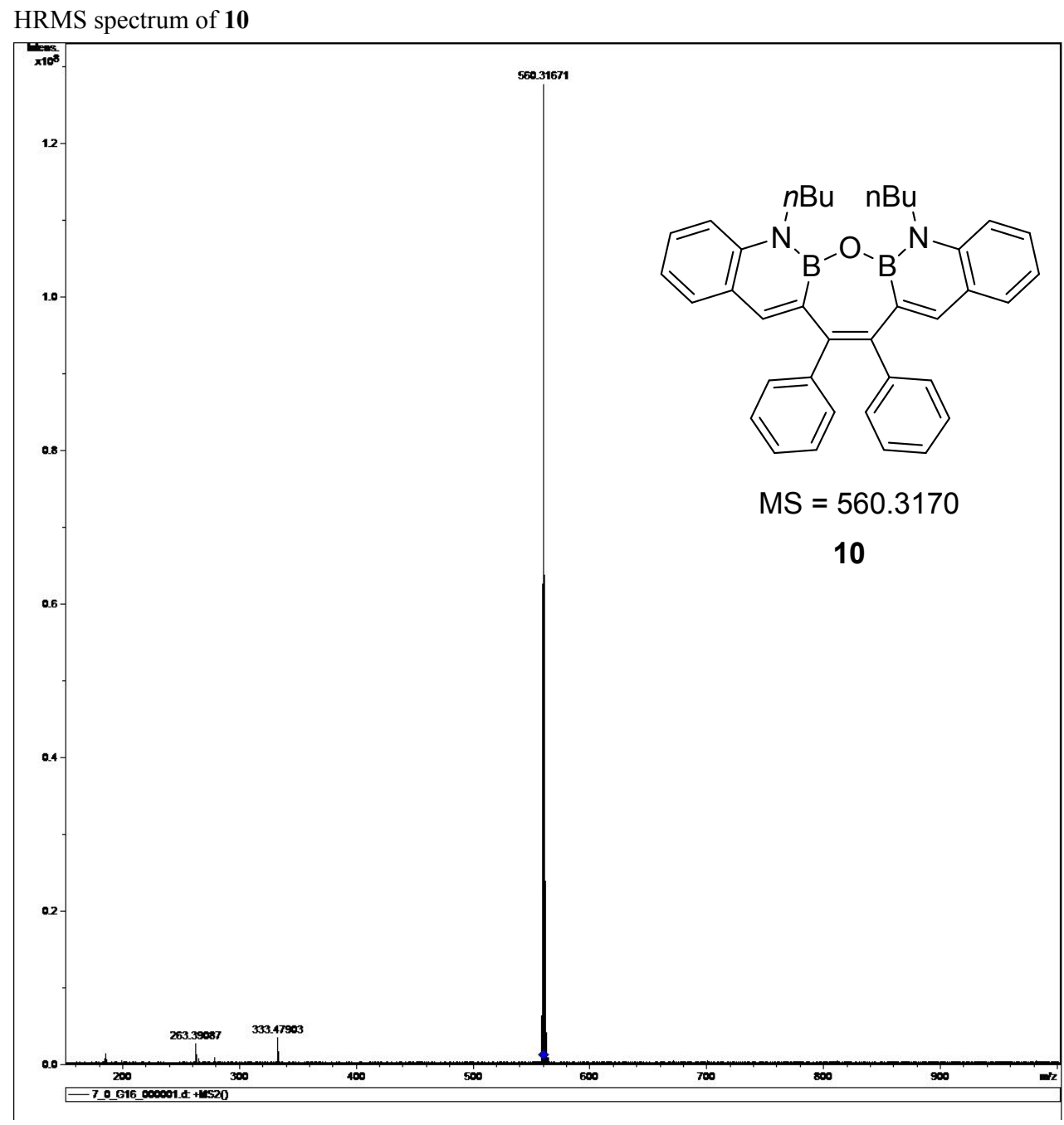

${ }^{1} \mathrm{H}$ - NMR spectrum of $\mathbf{1 1}$

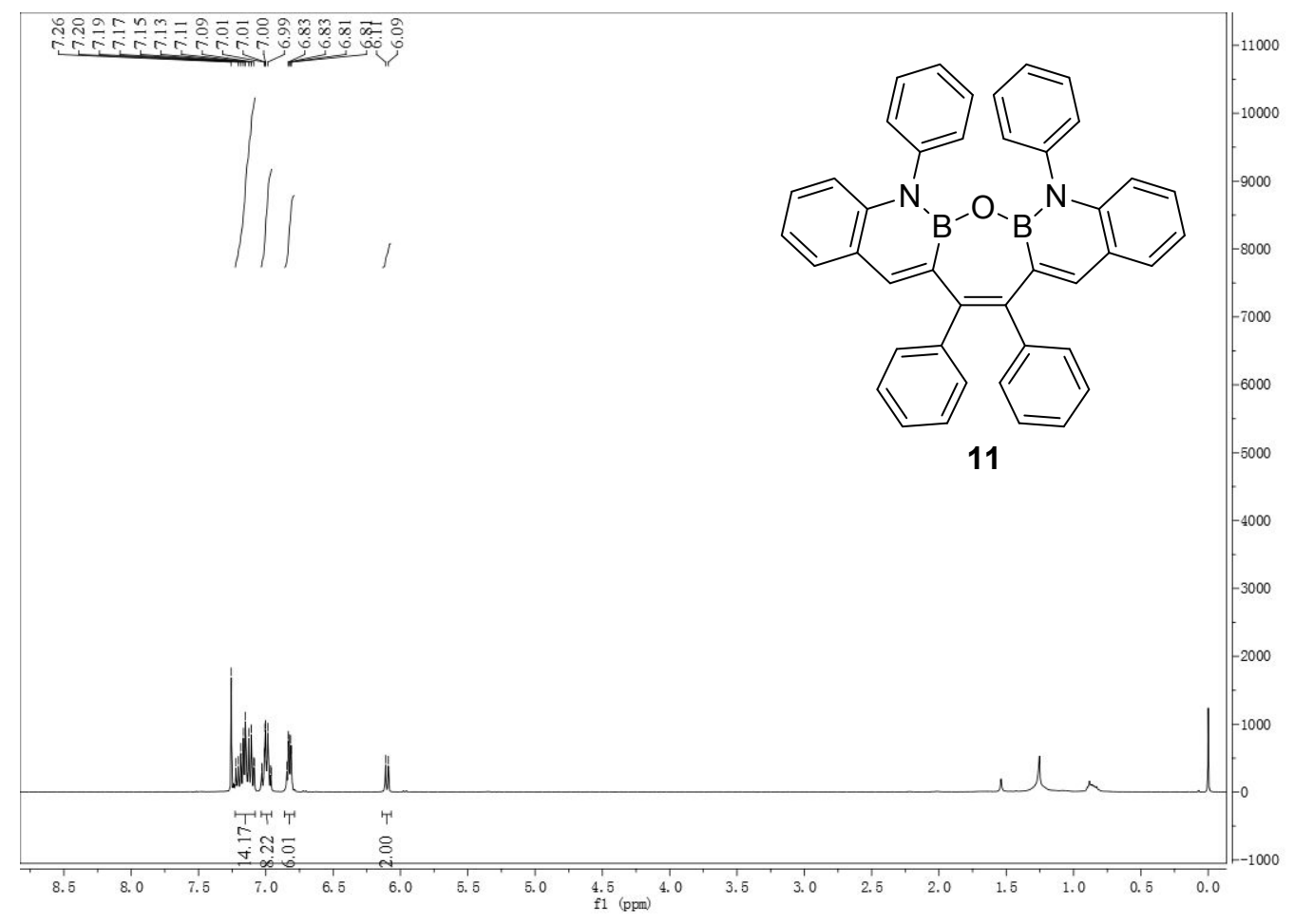




$$
u
$$


HRMS spectrum of $\mathbf{1 1}$

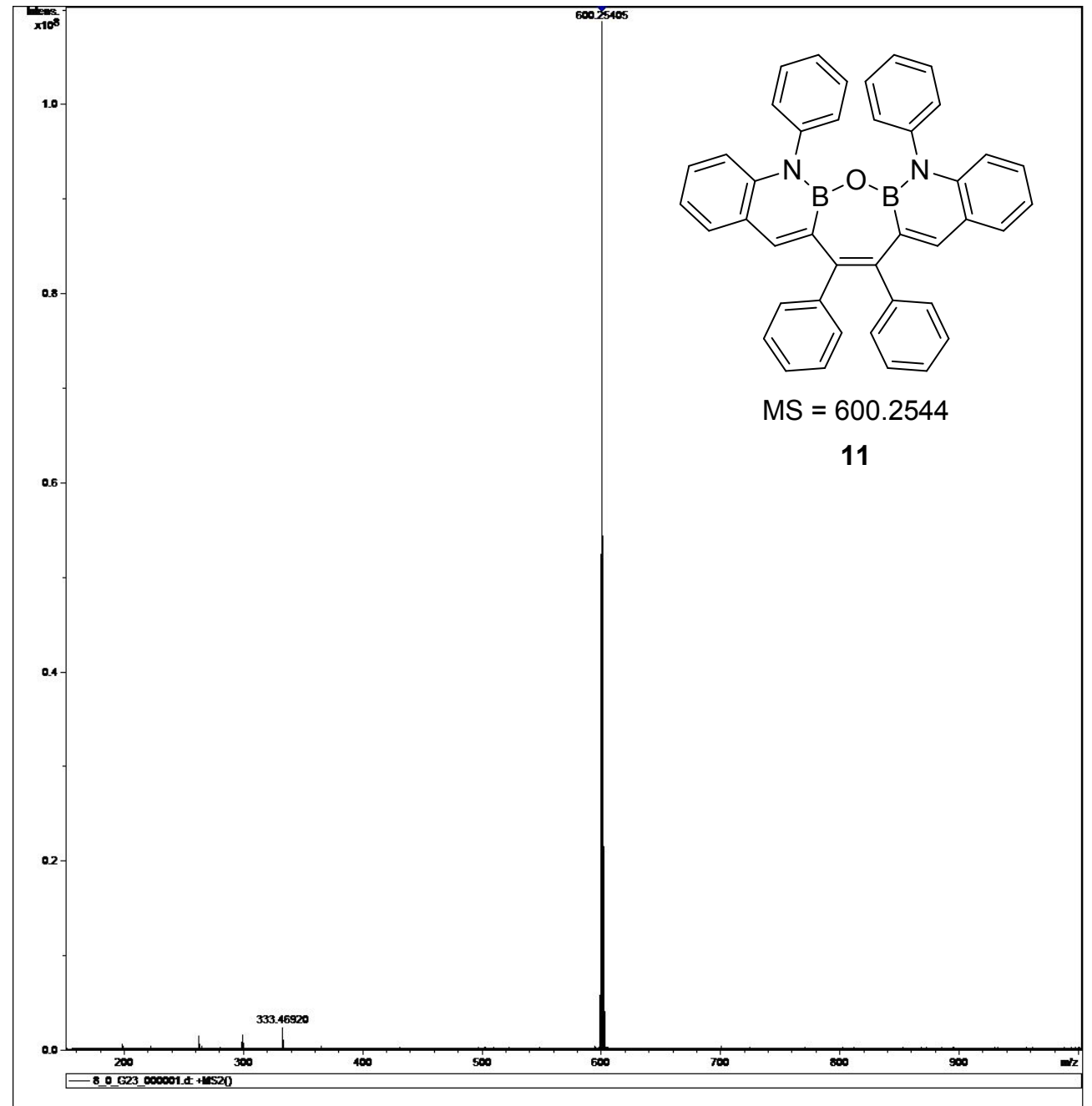

${ }^{1} \mathrm{H}$ - NMR spectrum of $\mathbf{1 2}$

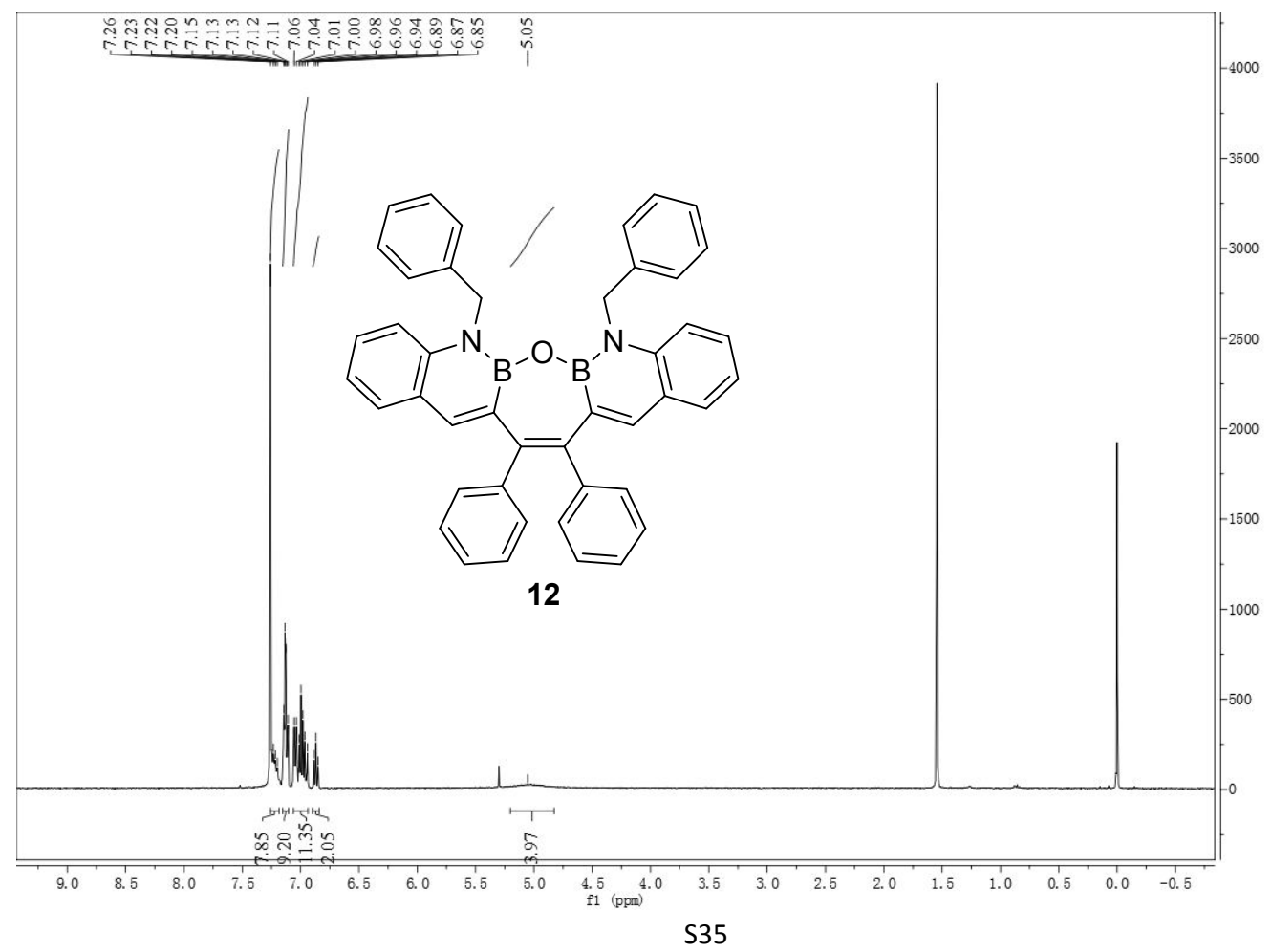


${ }^{13} \mathrm{C}$ - NMR spectrum of $\mathbf{1 2}$

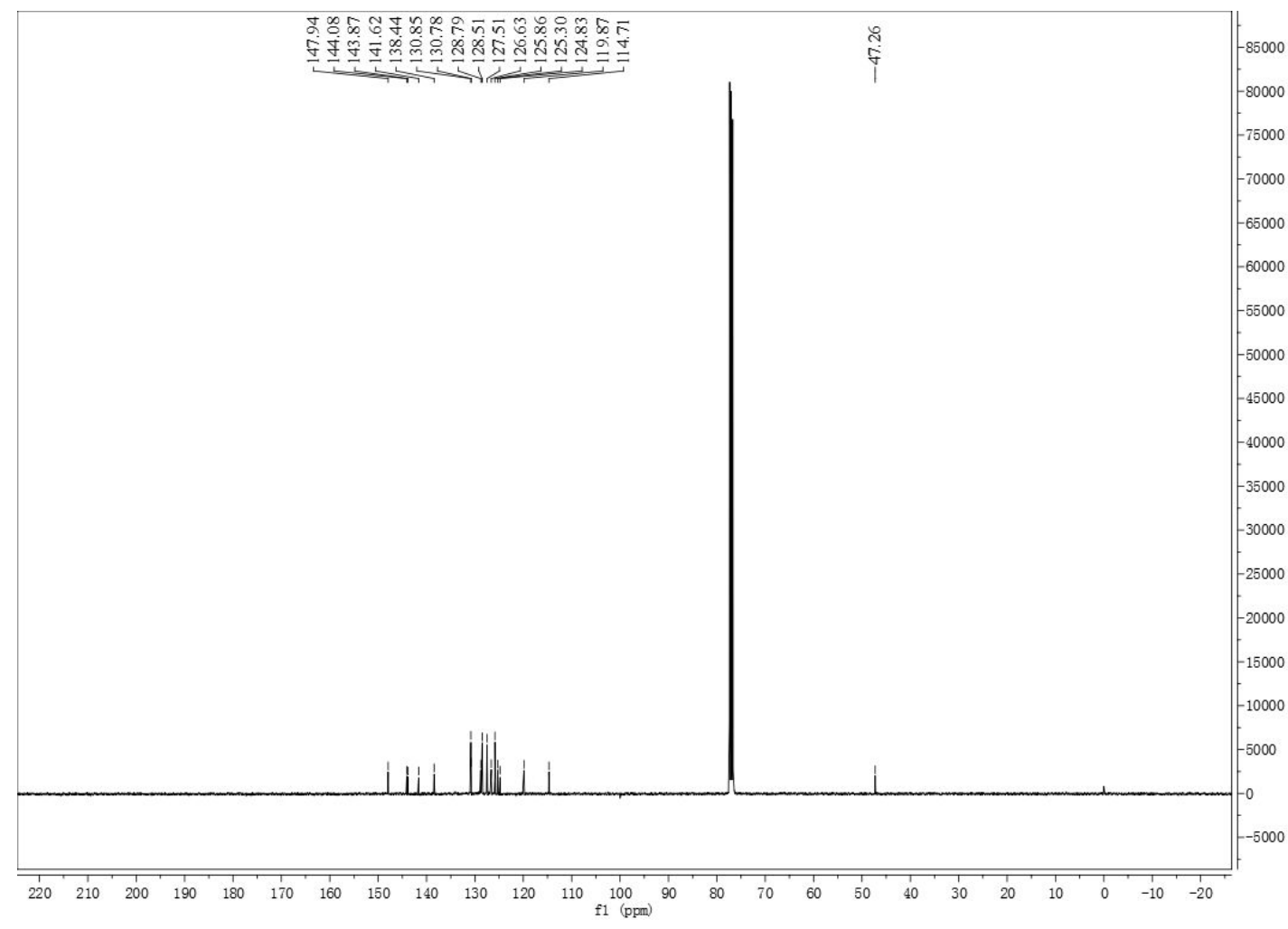

${ }^{11} \mathrm{~B}$ - NMR spectrum of $\mathbf{1 2}$

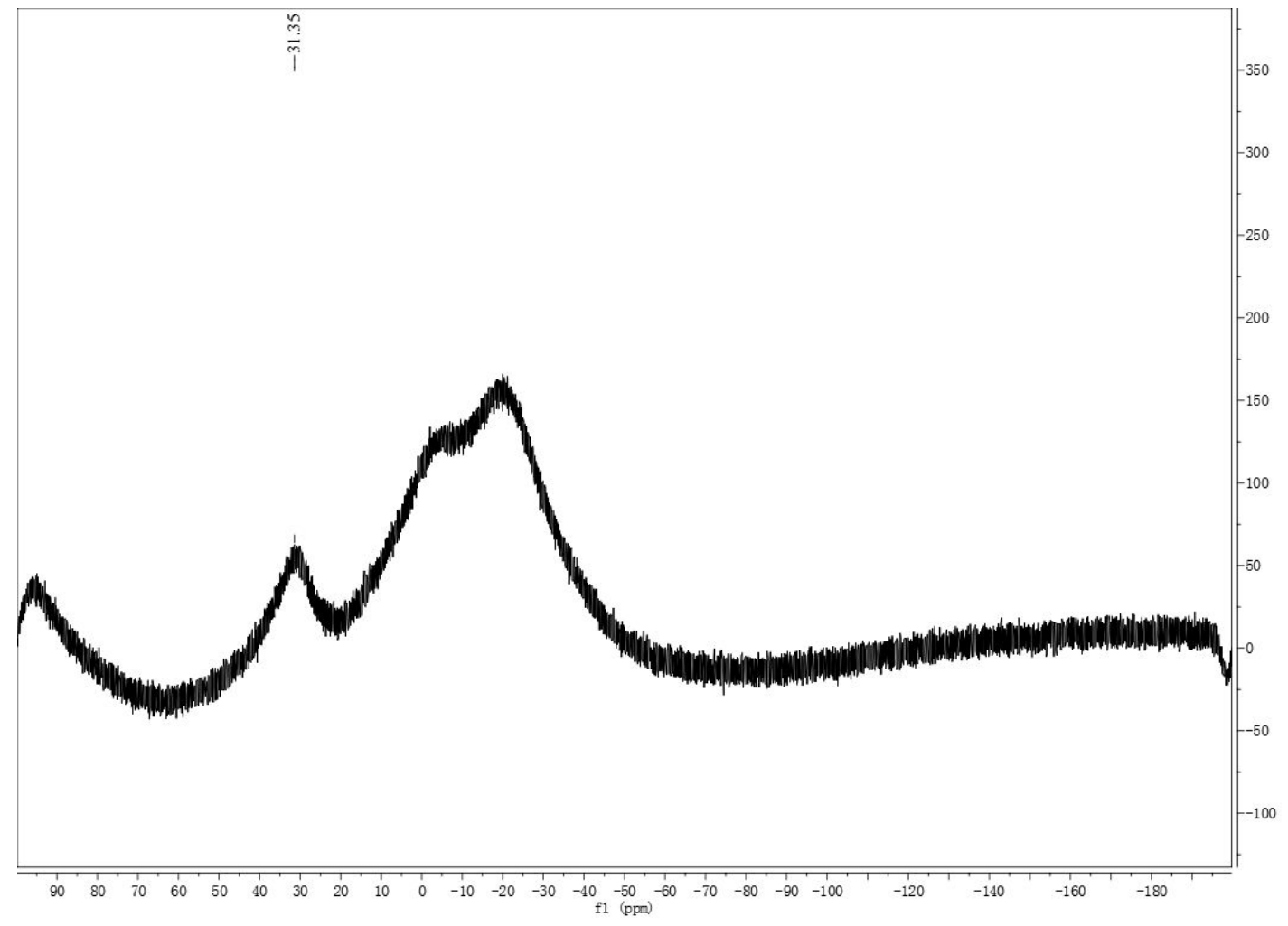


HRMS spectrum of $\mathbf{1 2}$

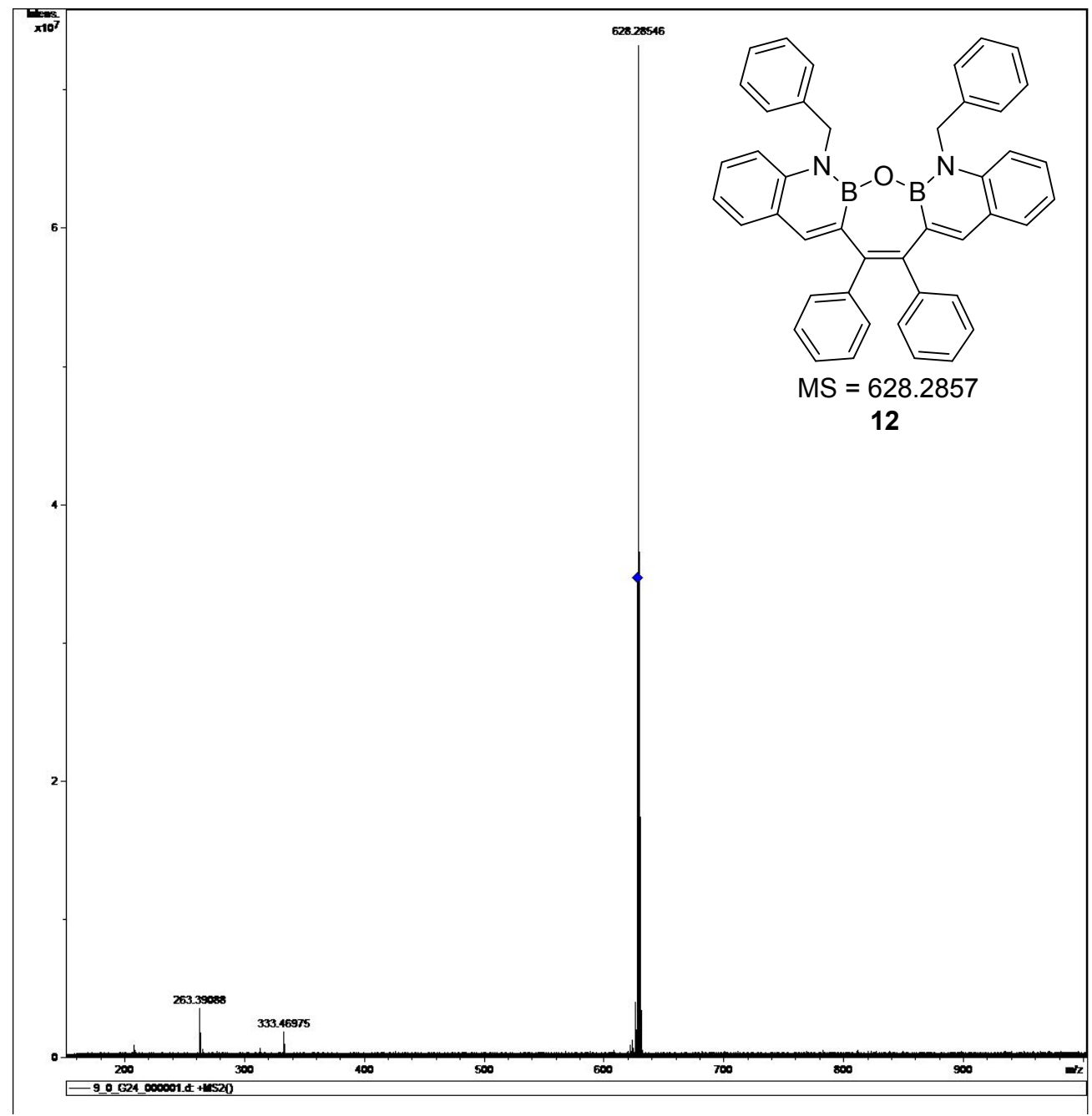

${ }^{1} \mathrm{H}$ - NMR spectrum of $\mathbf{1 3}$

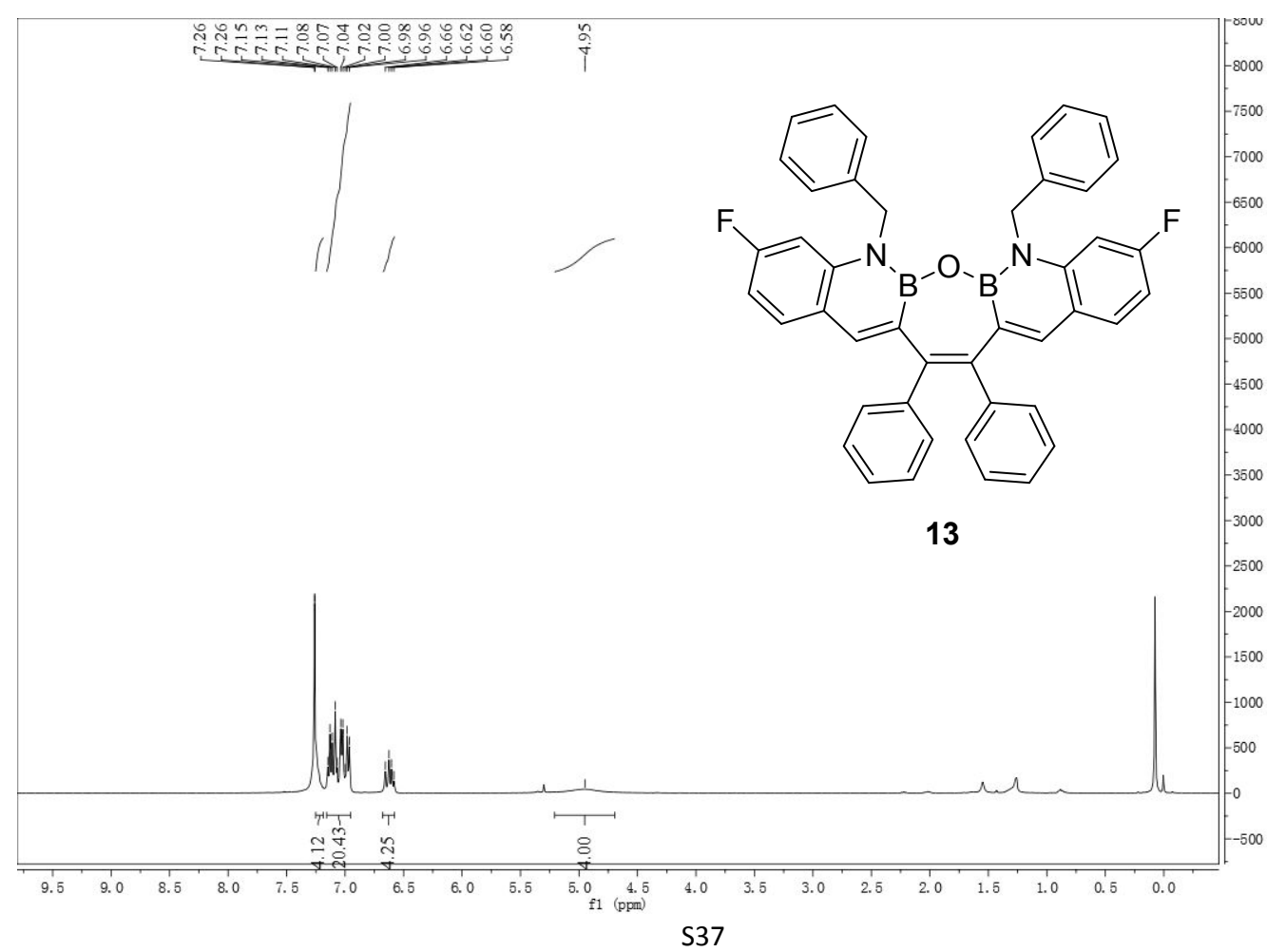


${ }^{13} \mathrm{C}$ - NMR spectrum of $\mathbf{1 3}$

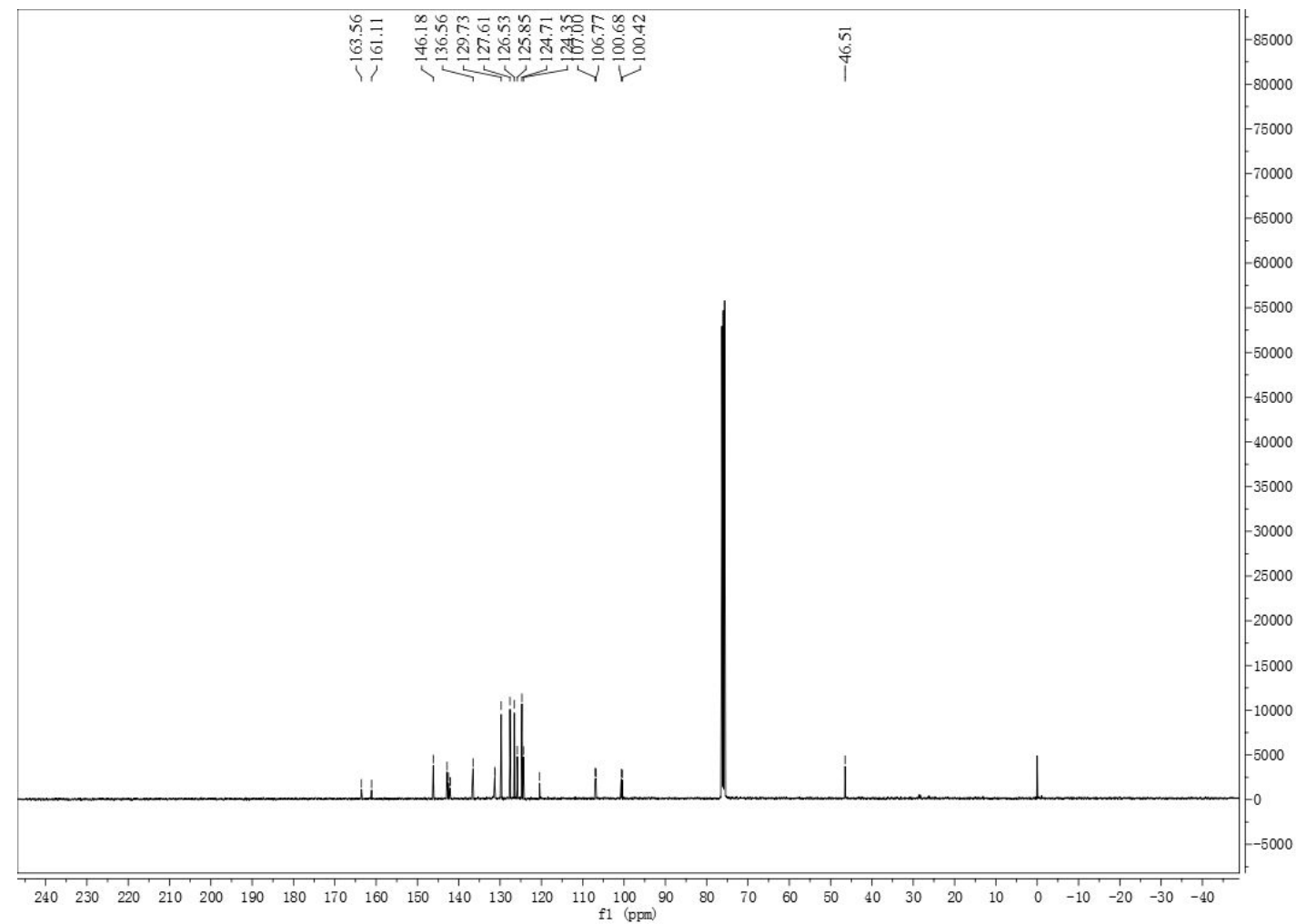

${ }^{11} \mathrm{~B}$ - NMR spectrum of $\mathbf{1 3}$

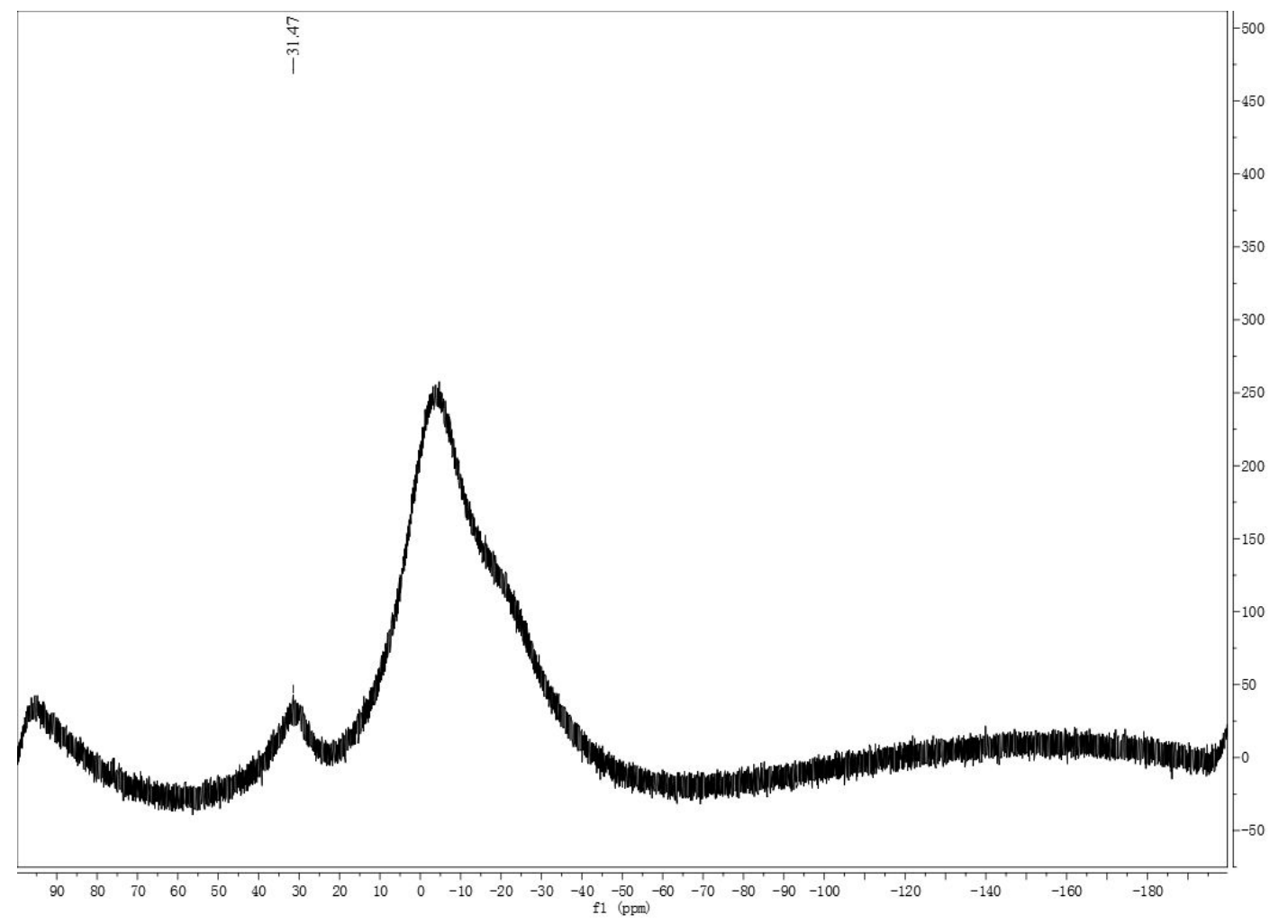


${ }^{19} \mathrm{~F}$ - NMR spectrum of $\mathbf{1 3}$

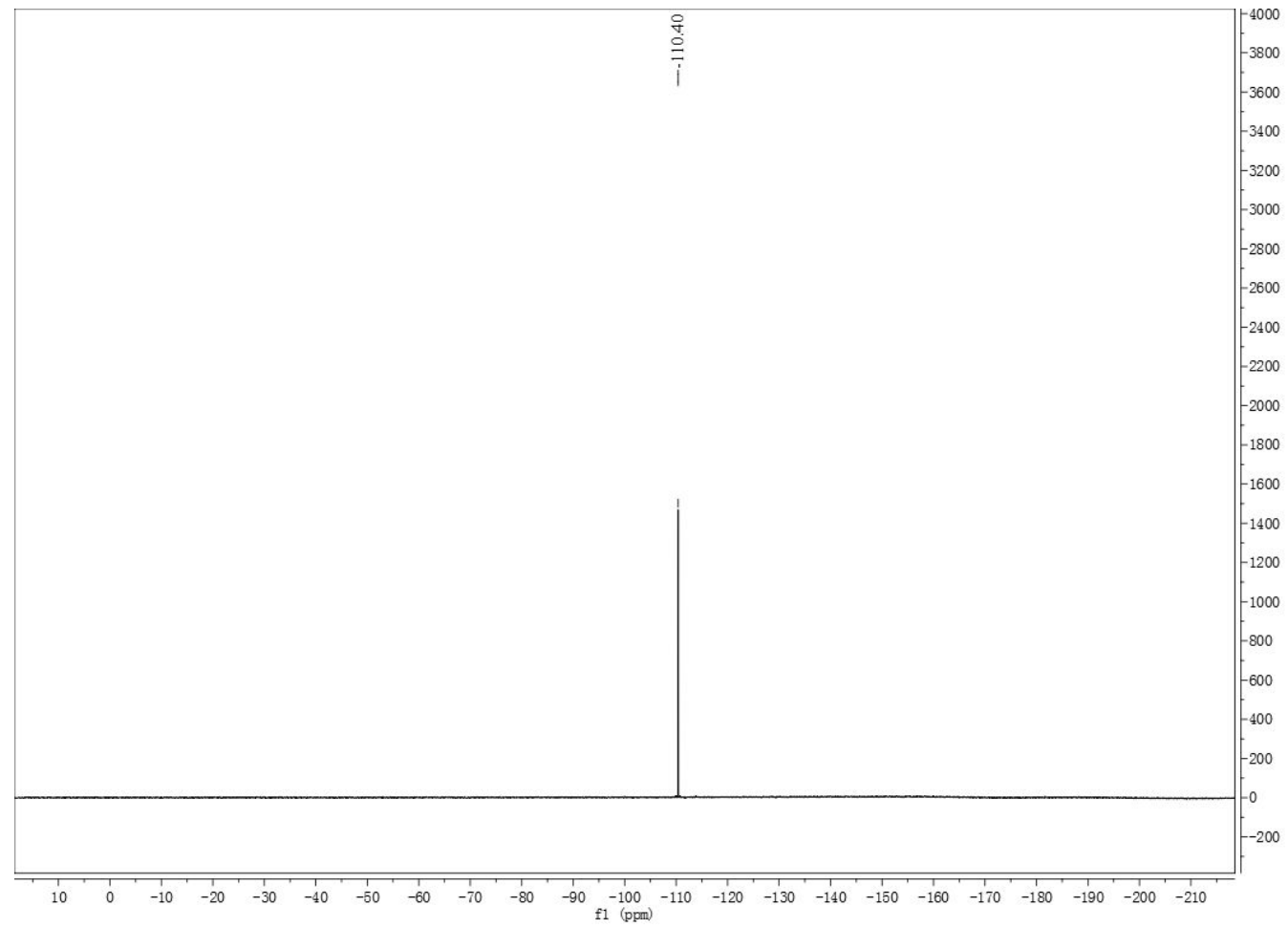

HRMS spectrum of $\mathbf{1 3}$

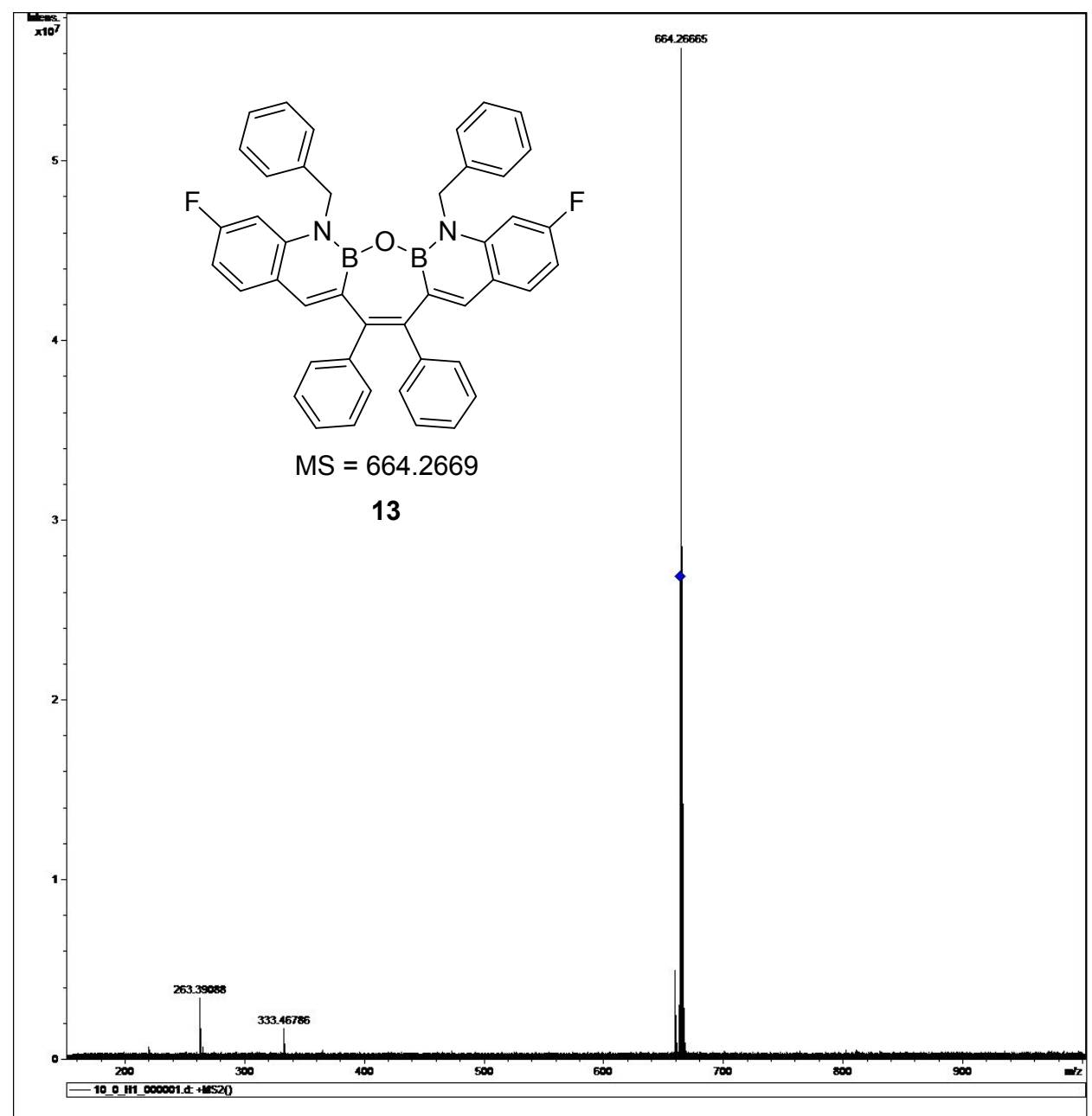


${ }^{1} \mathrm{H}$ - NMR spectrum of $\mathbf{1 4}$

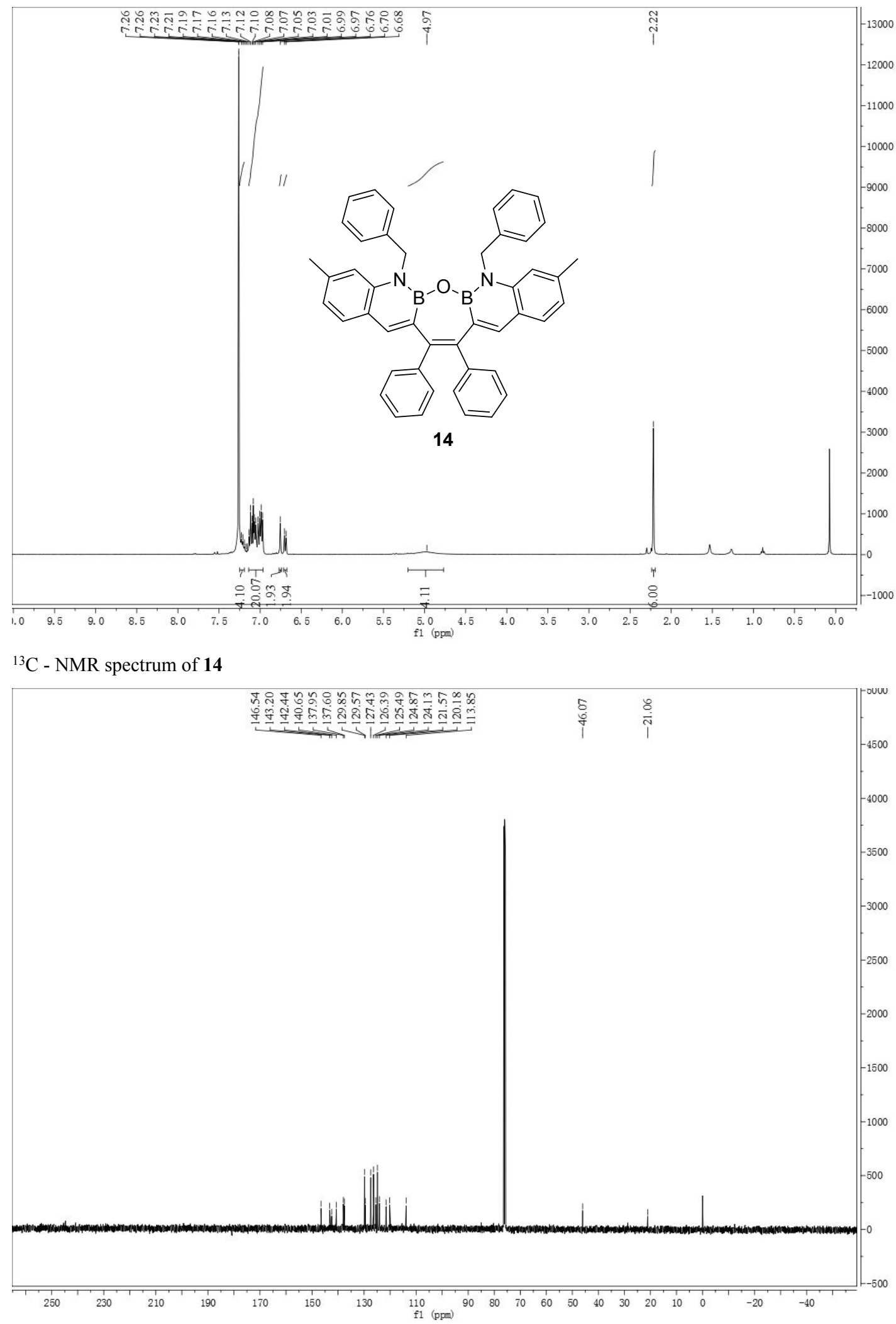


${ }^{11} \mathrm{~B}$ - NMR spectrum of $\mathbf{1 4}$

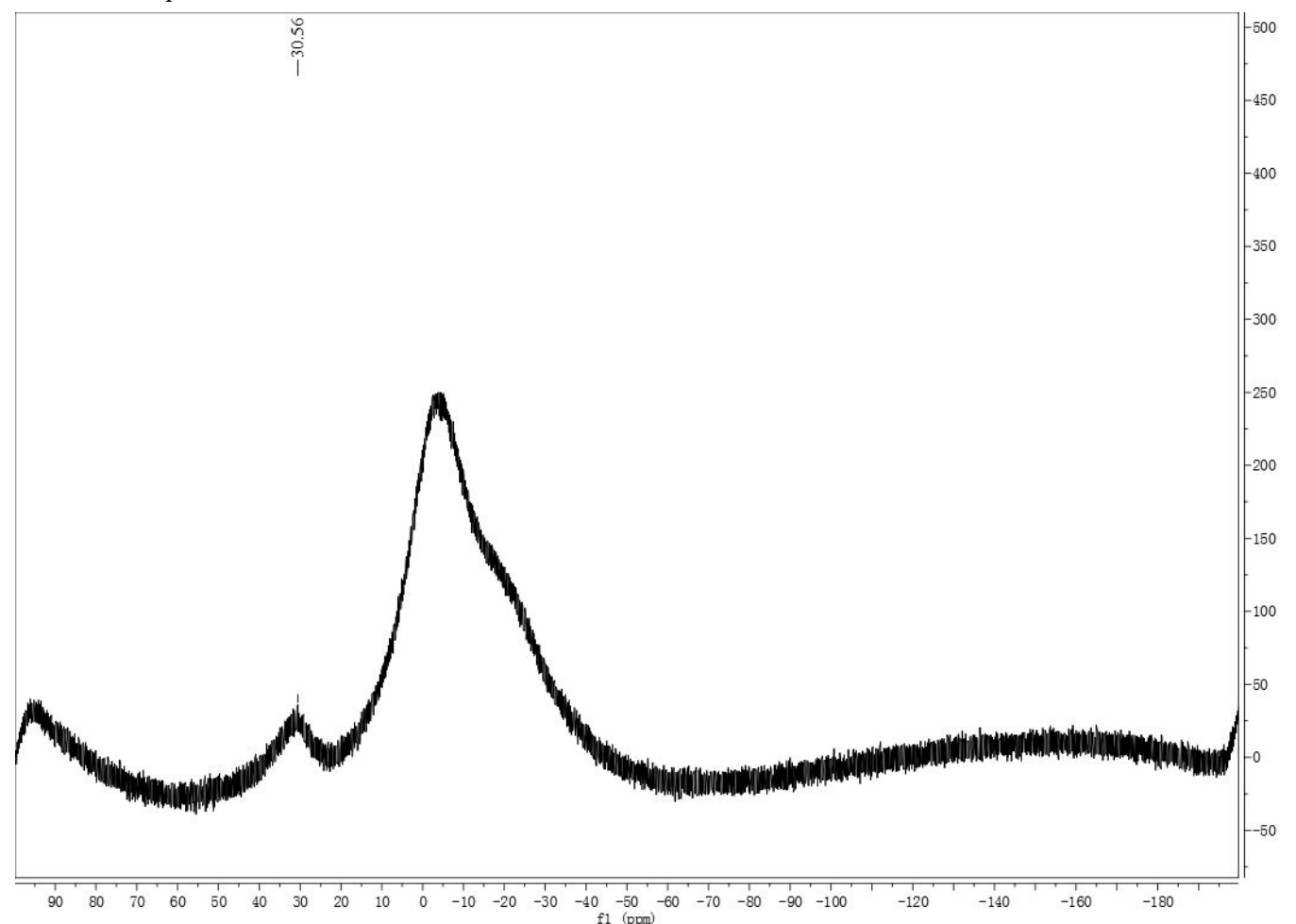

HRMS spectrum of $\mathbf{1 4}$

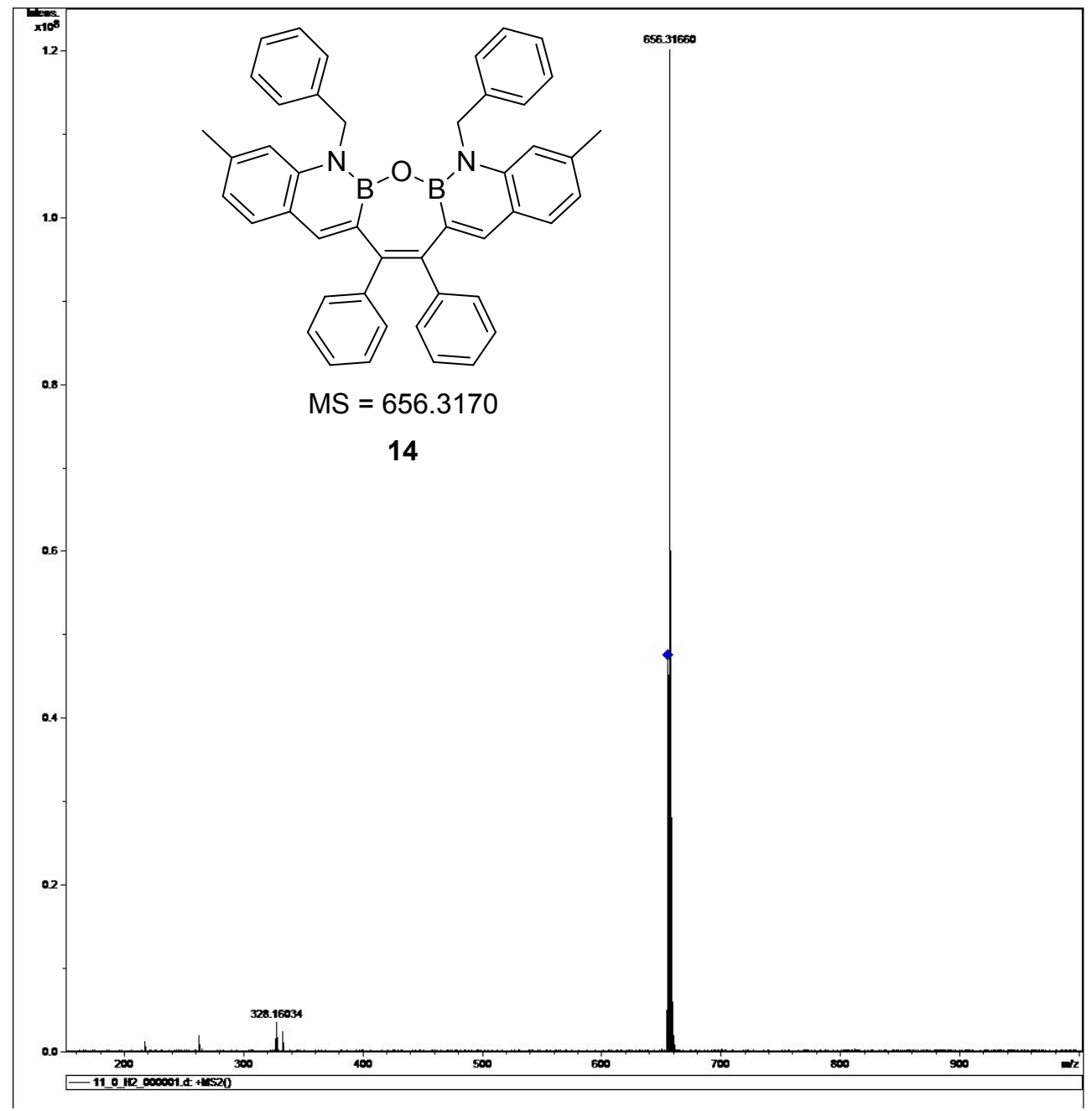


${ }^{1} \mathrm{H}$ - NMR spectrum of $\mathbf{1 5}$

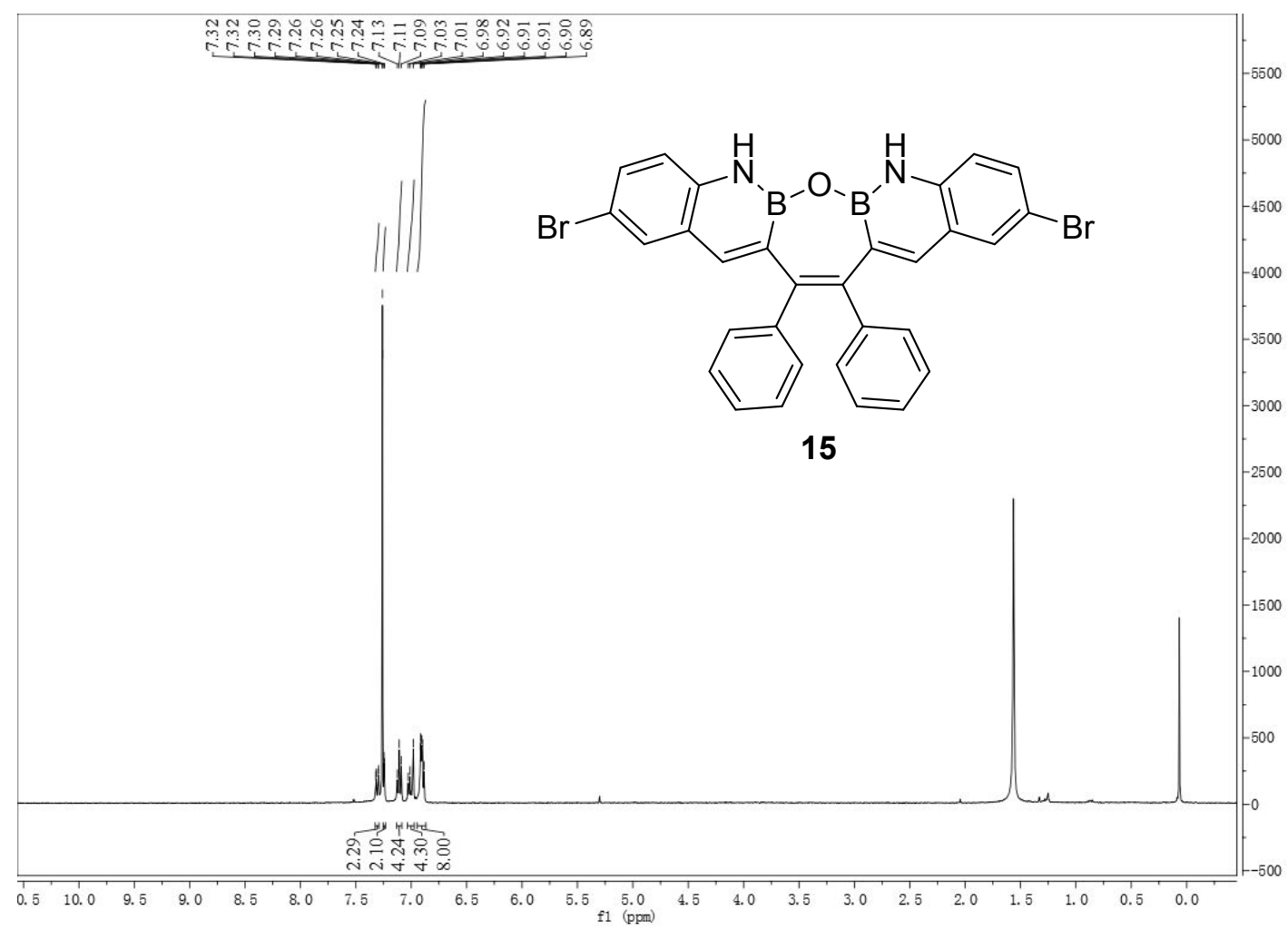

${ }^{13} \mathrm{C}$ - NMR spectrum of $\mathbf{1 5}$

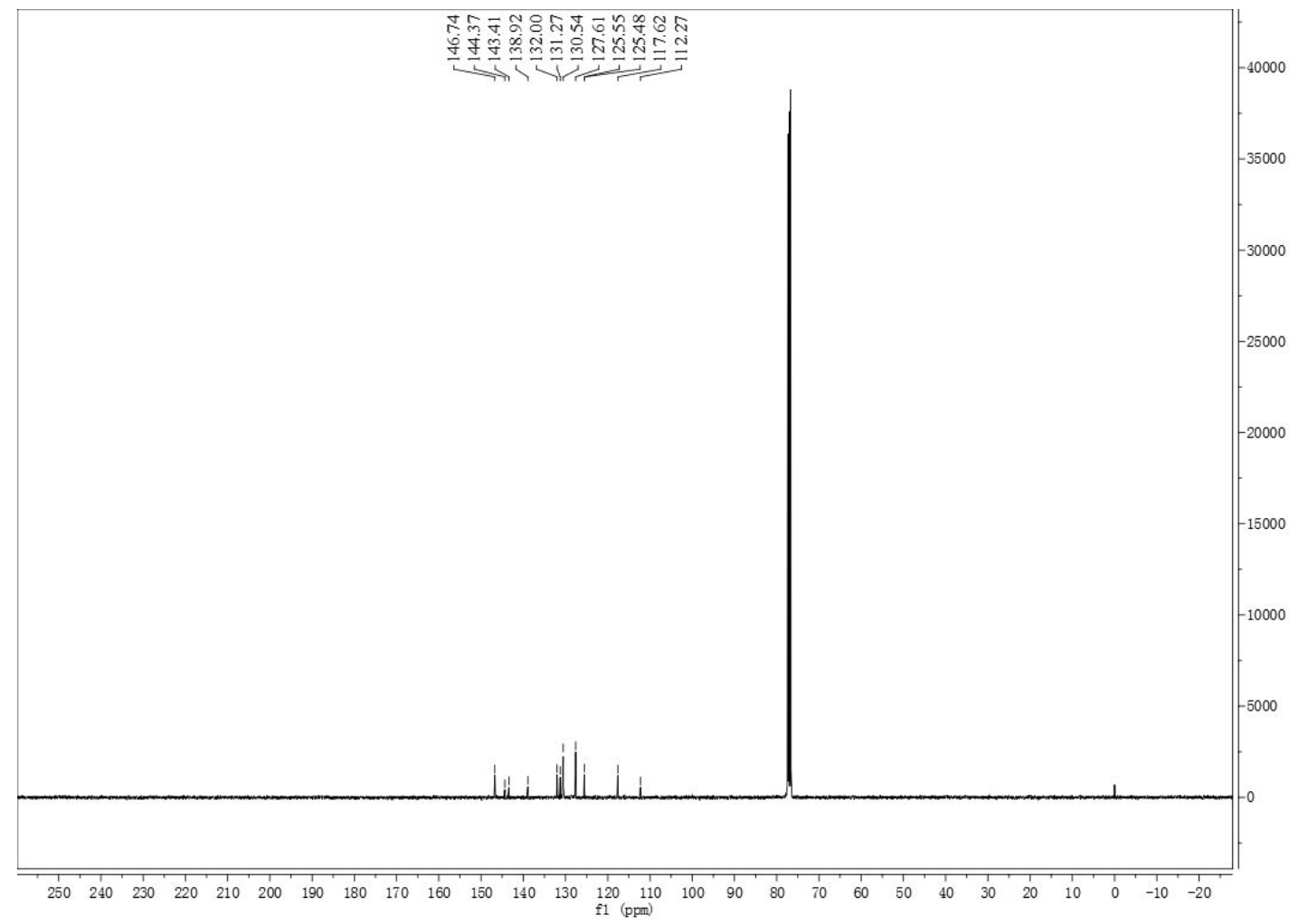


${ }^{11} \mathrm{~B}$ - NMR spectrum of $\mathbf{1 5}$

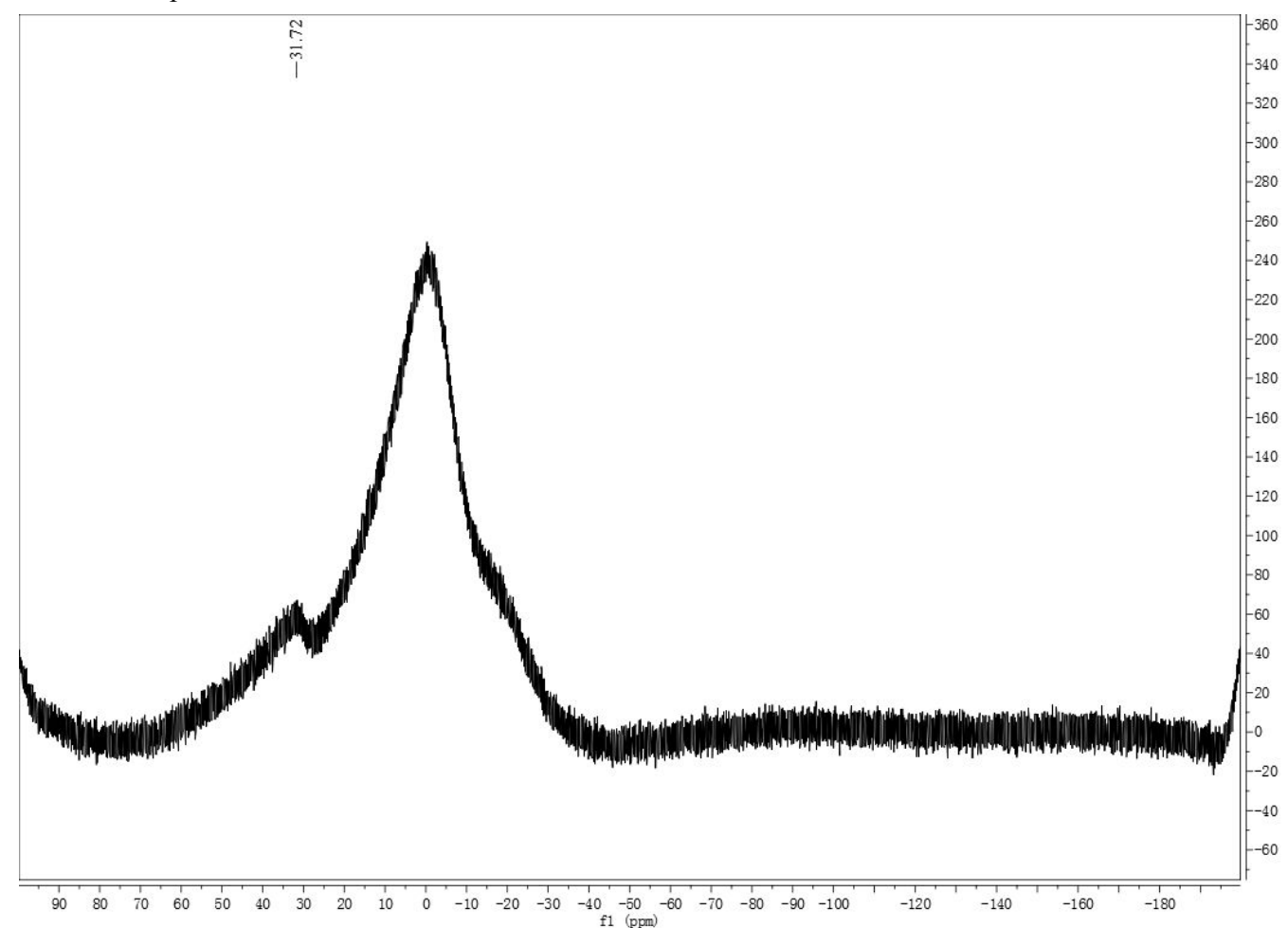

HRMS spectrum of $\mathbf{1 5}$

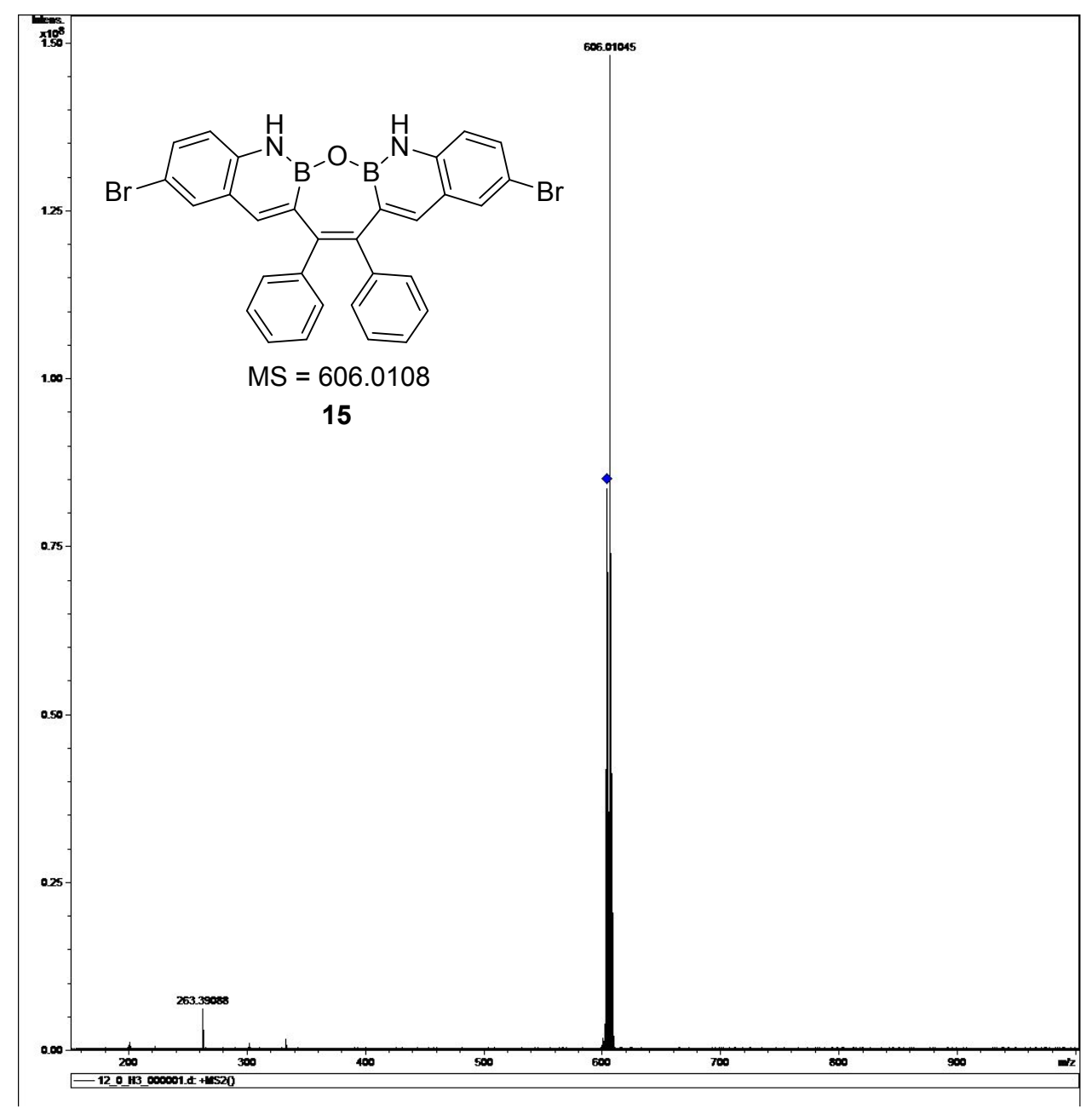


${ }^{1} \mathrm{H}$ - NMR spectrum of $\mathbf{1 6}$

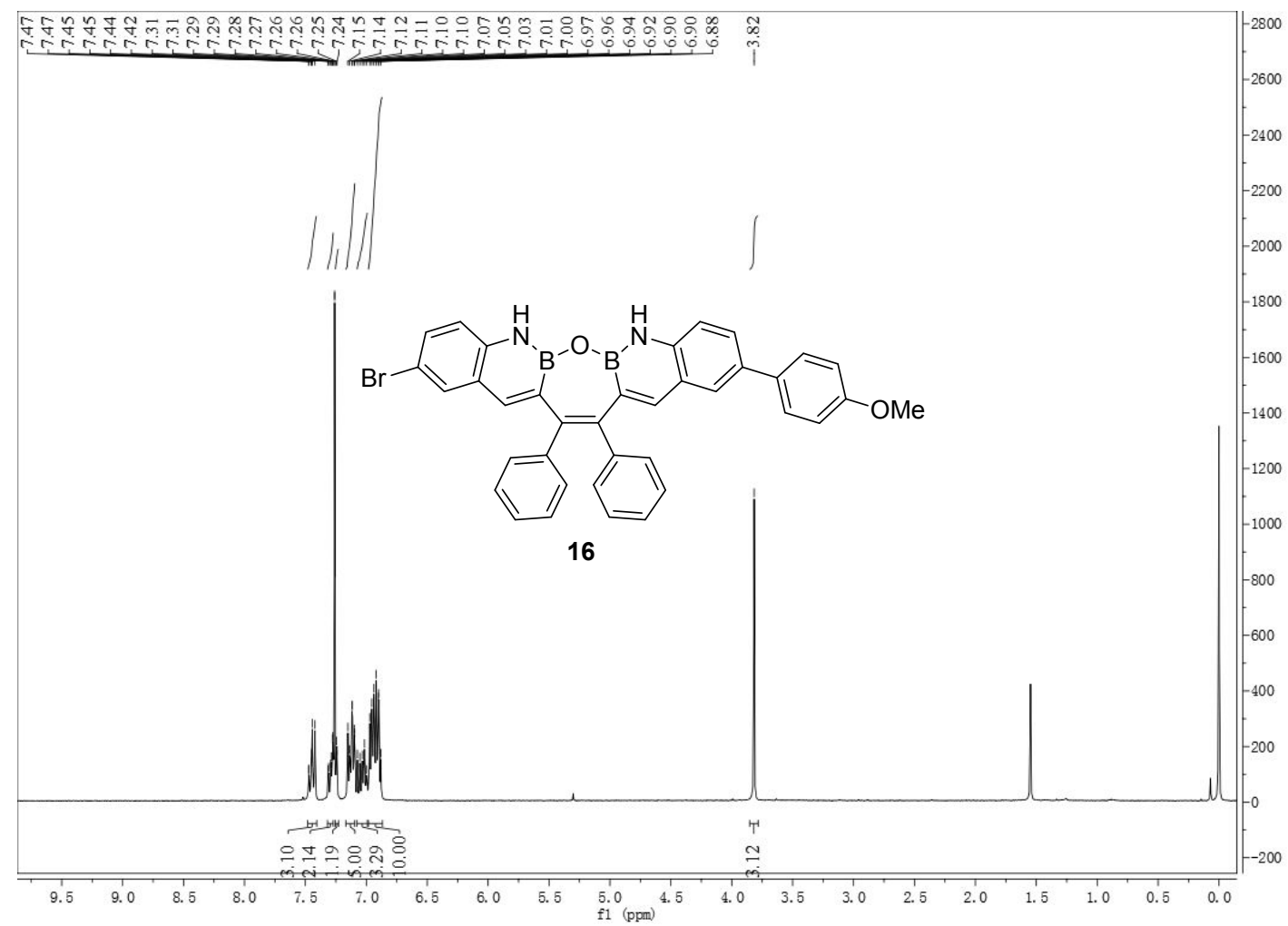

${ }^{13} \mathrm{C}$ - NMR spectrum of $\mathbf{1 6}$

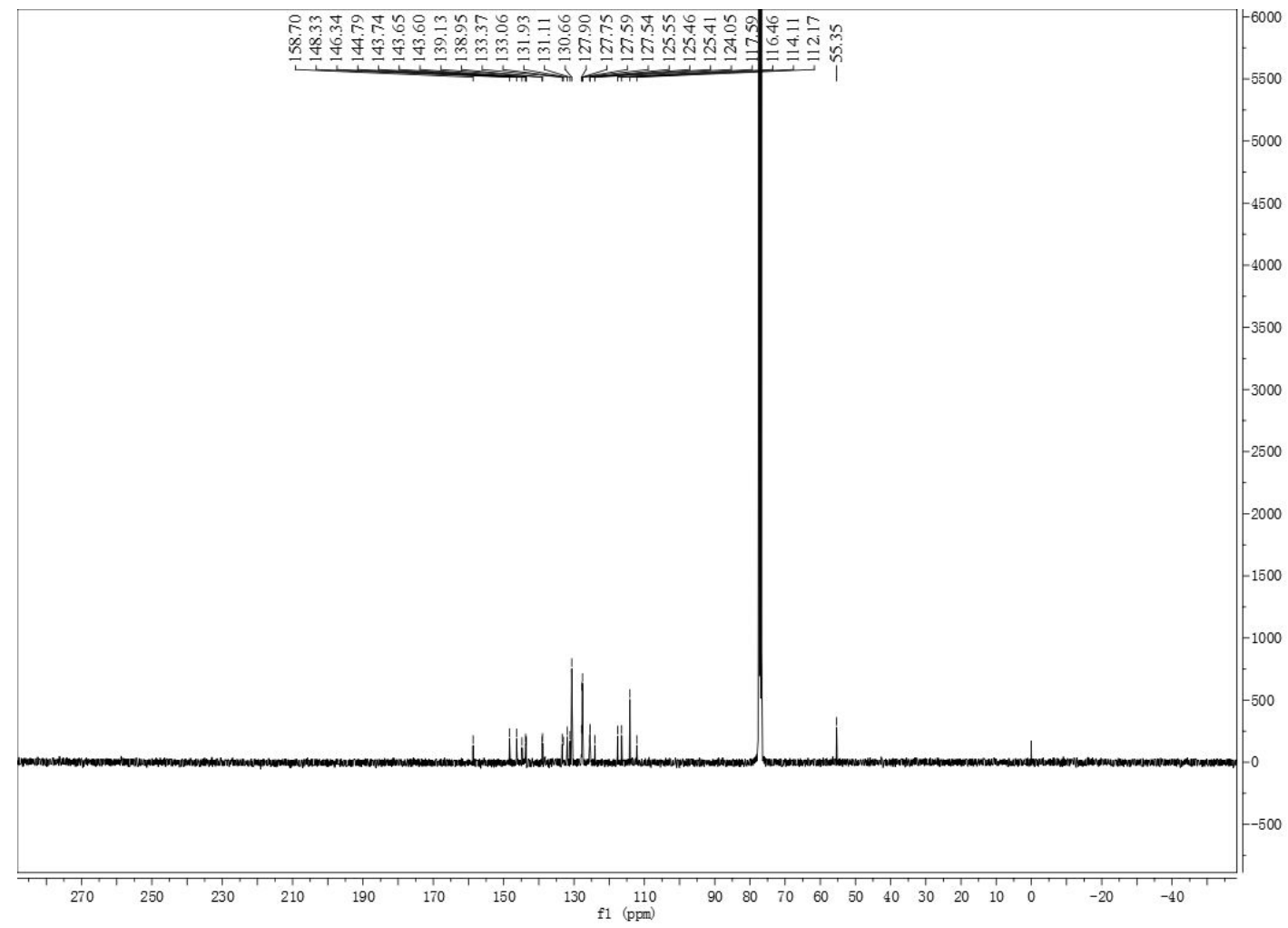


${ }^{11} \mathrm{~B}$ - NMR spectrum of $\mathbf{1 6}$

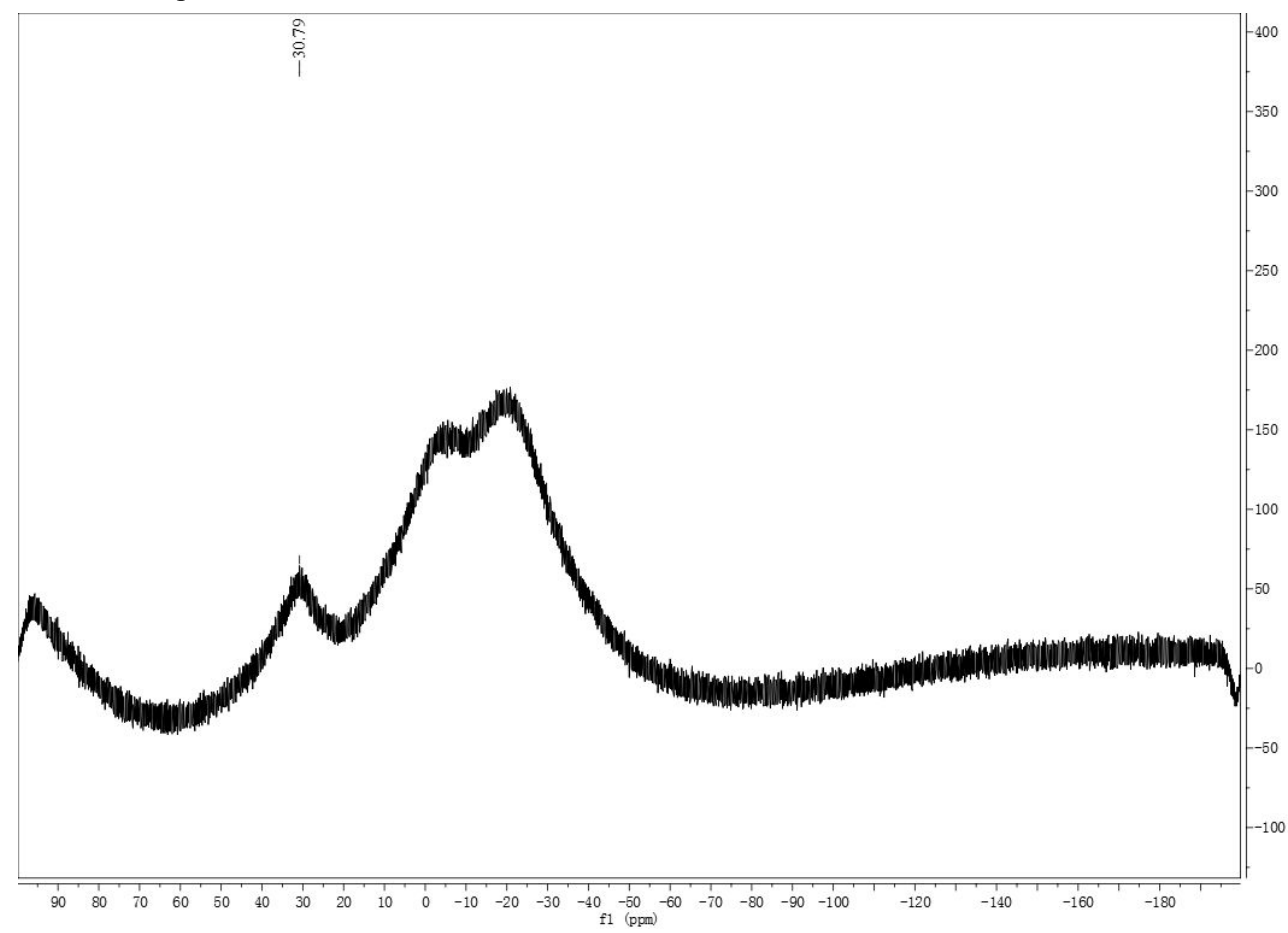

HRMS spectrum of $\mathbf{1 6}$

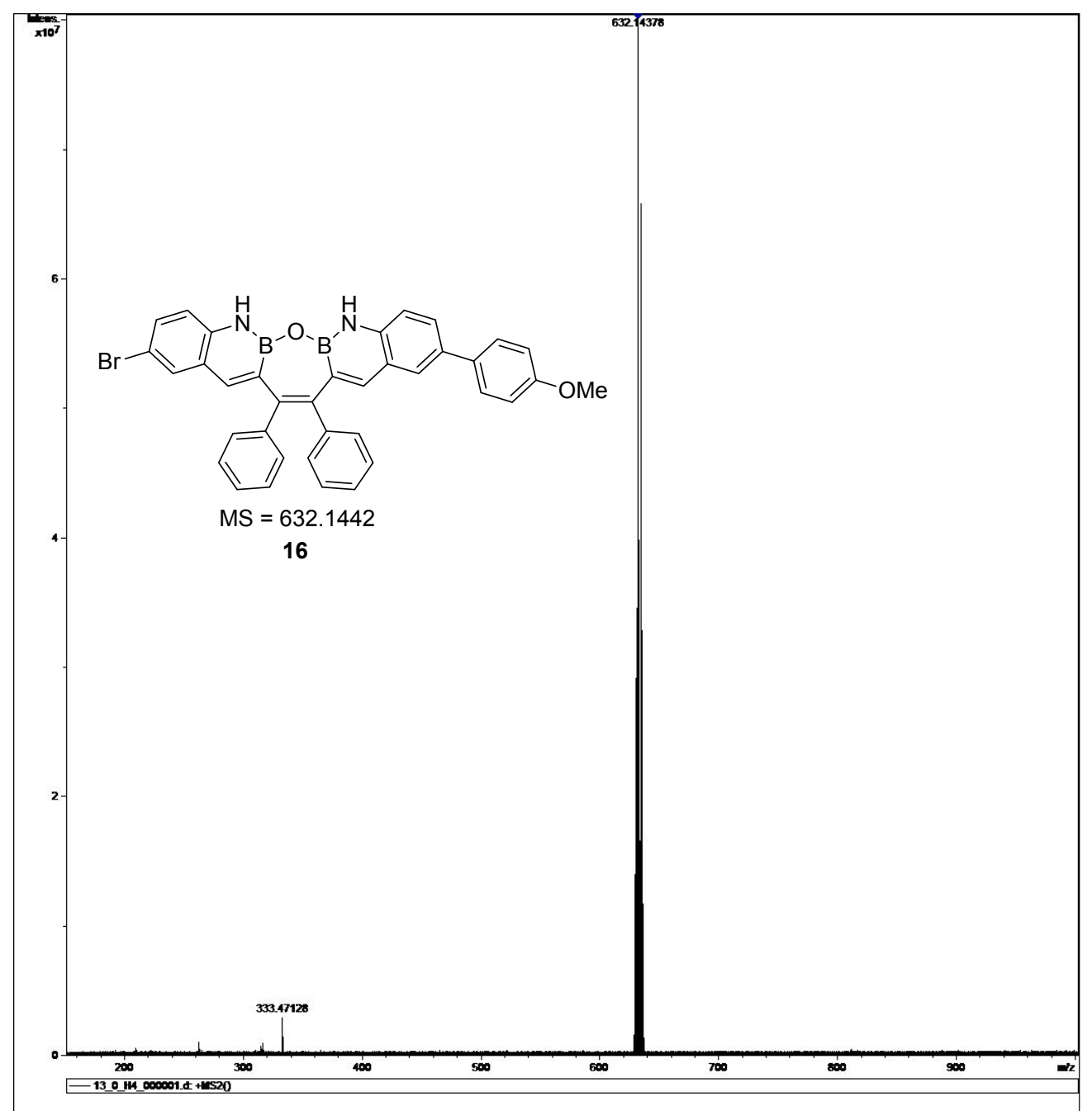


${ }^{1} \mathrm{H}$ - NMR spectrum of $\mathbf{1 7}$

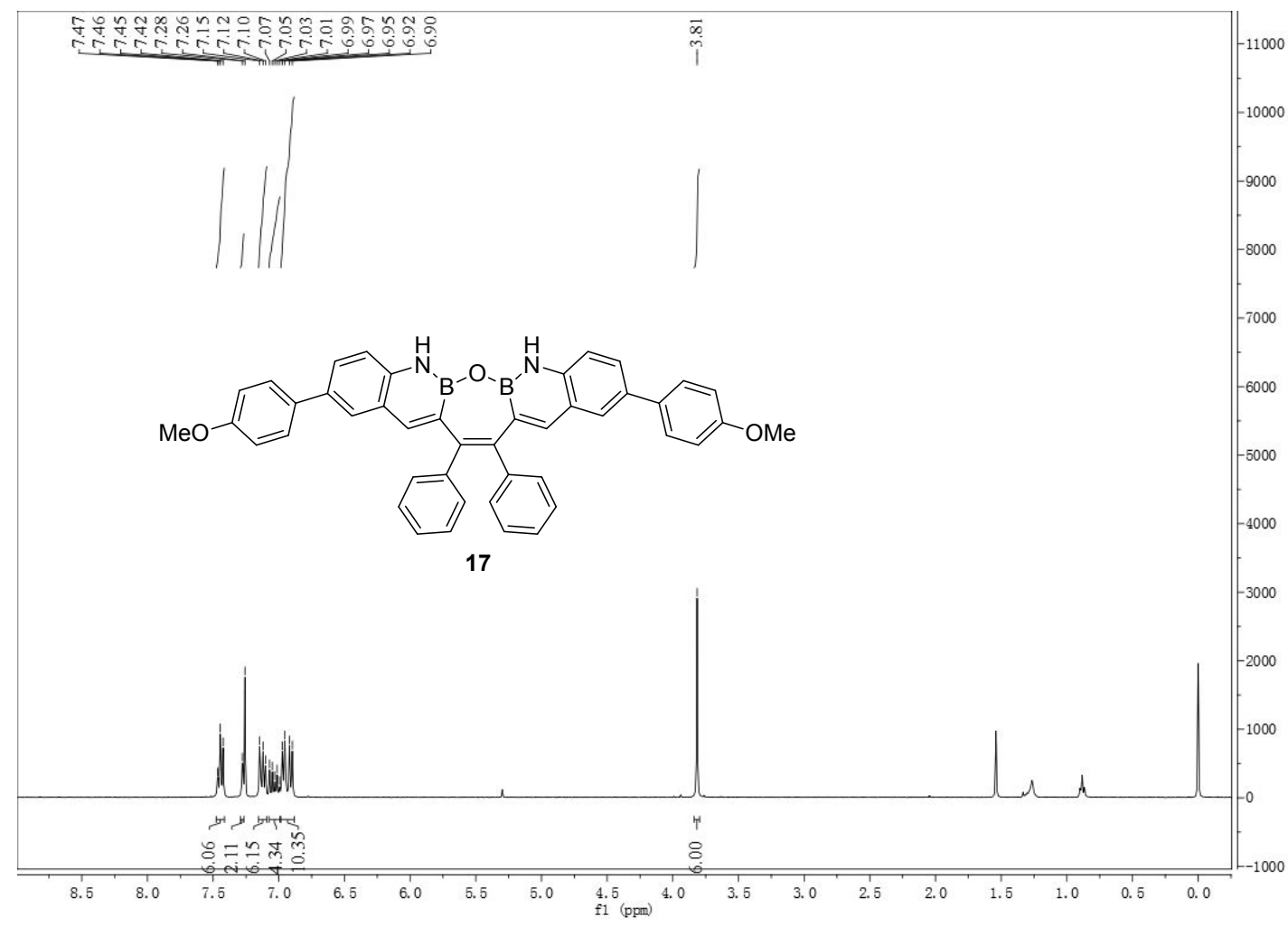

${ }^{13} \mathrm{C}$ - NMR spectrum of $\mathbf{1 7}$

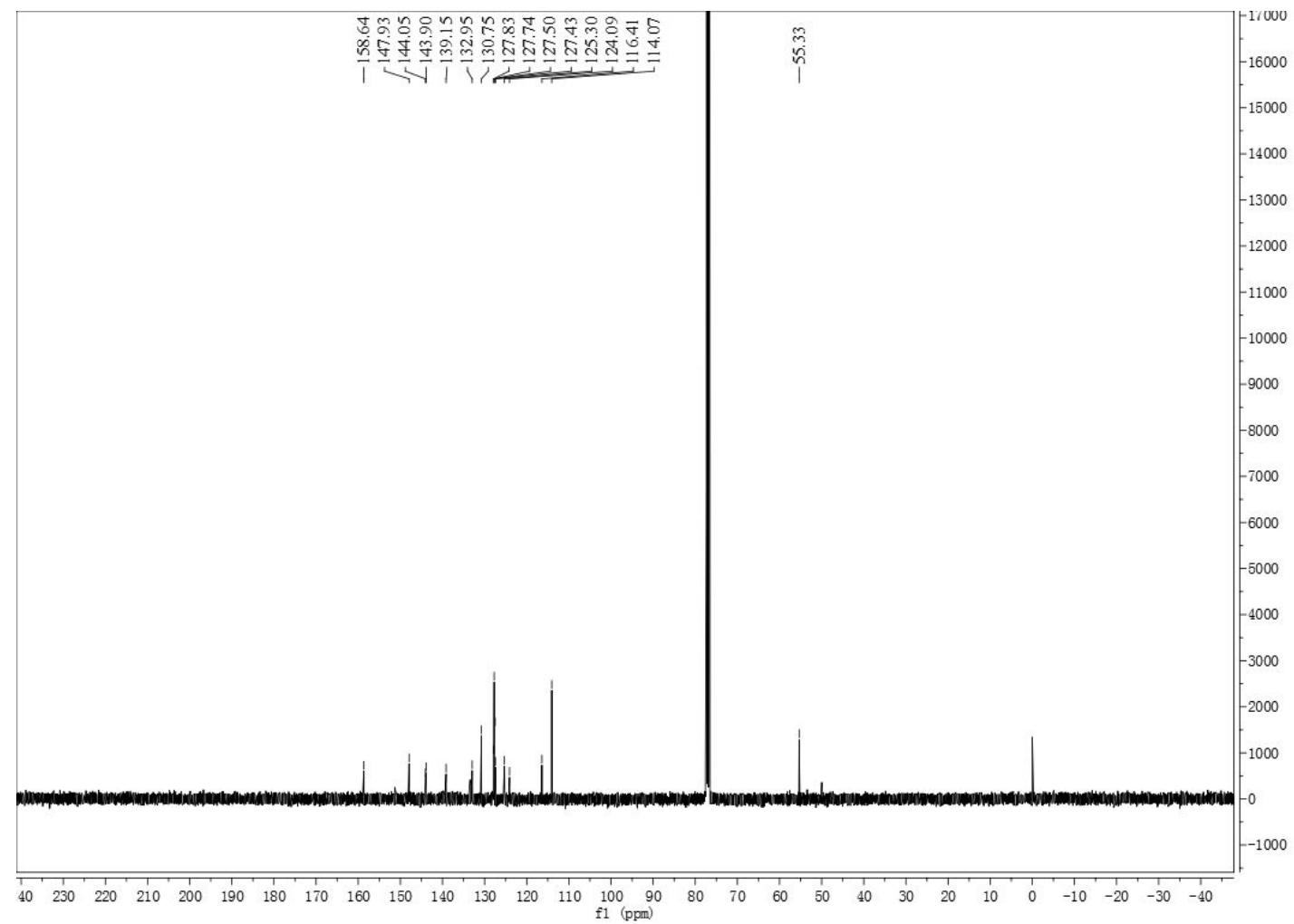


${ }^{11} \mathrm{~B}$ - NMR spectrum of $\mathbf{1 7}$

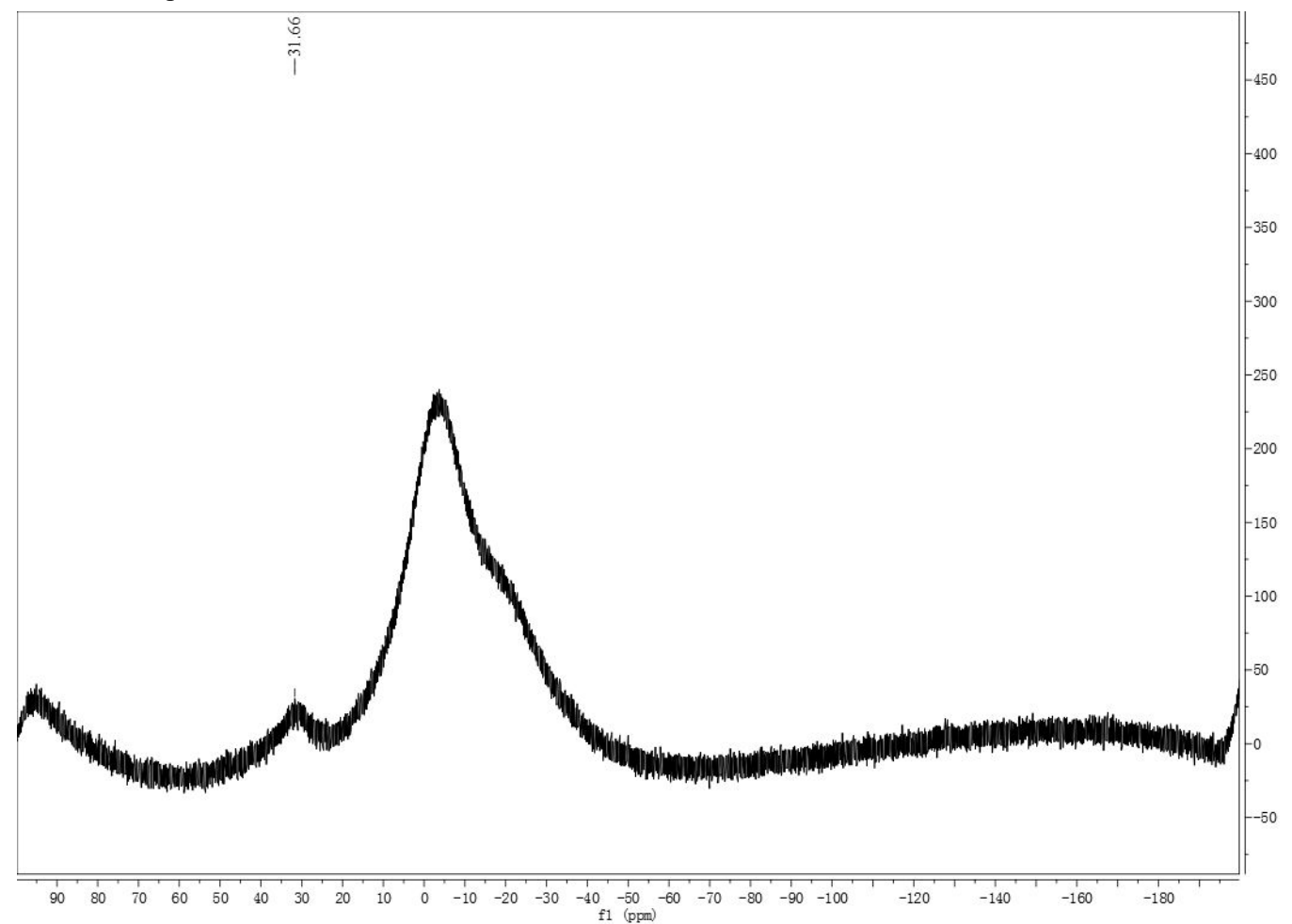

HRMS spectrum of $\mathbf{1 7}$

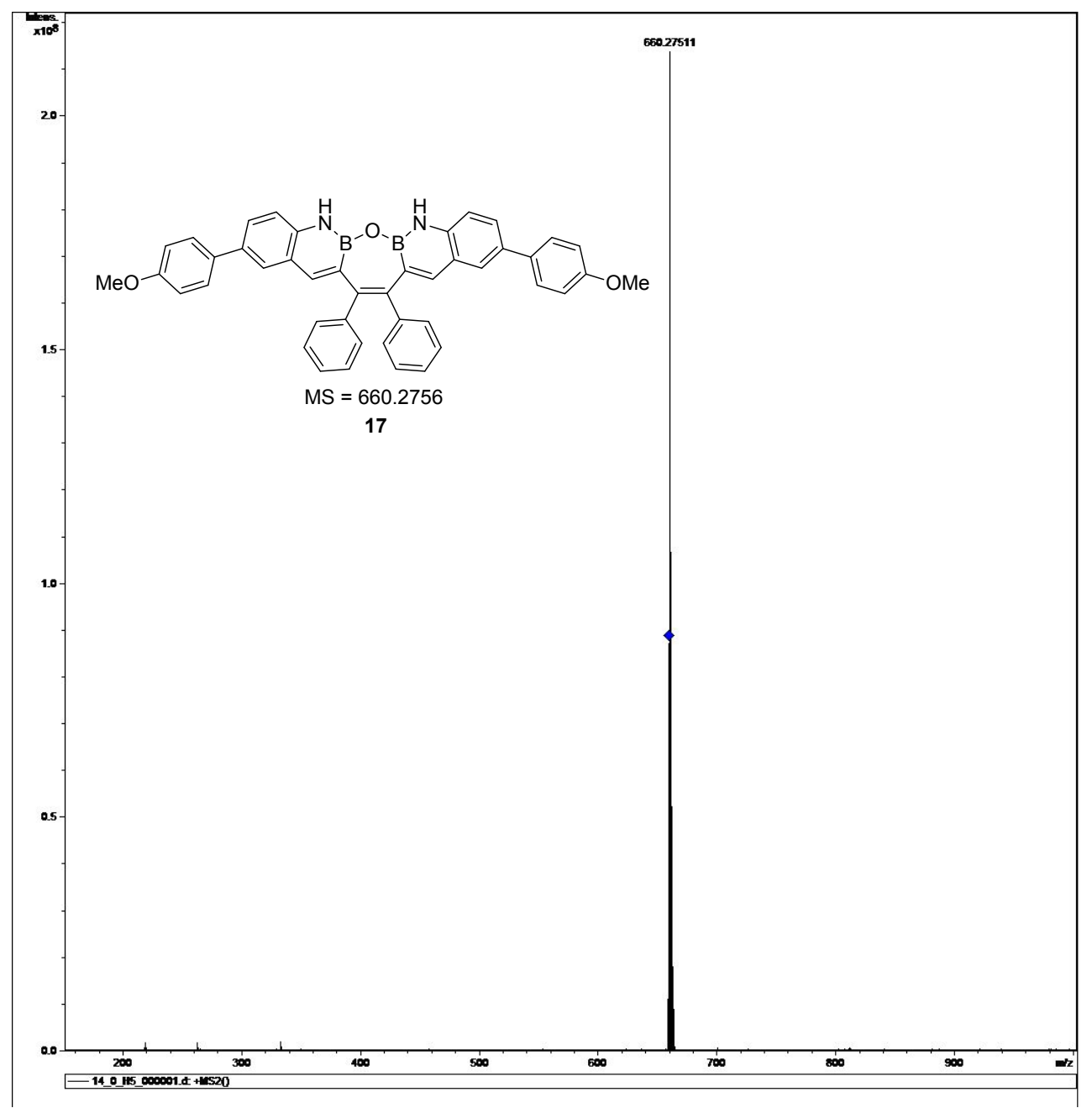


${ }^{1} \mathrm{H}$ - NMR spectrum of $\mathbf{2 c}$

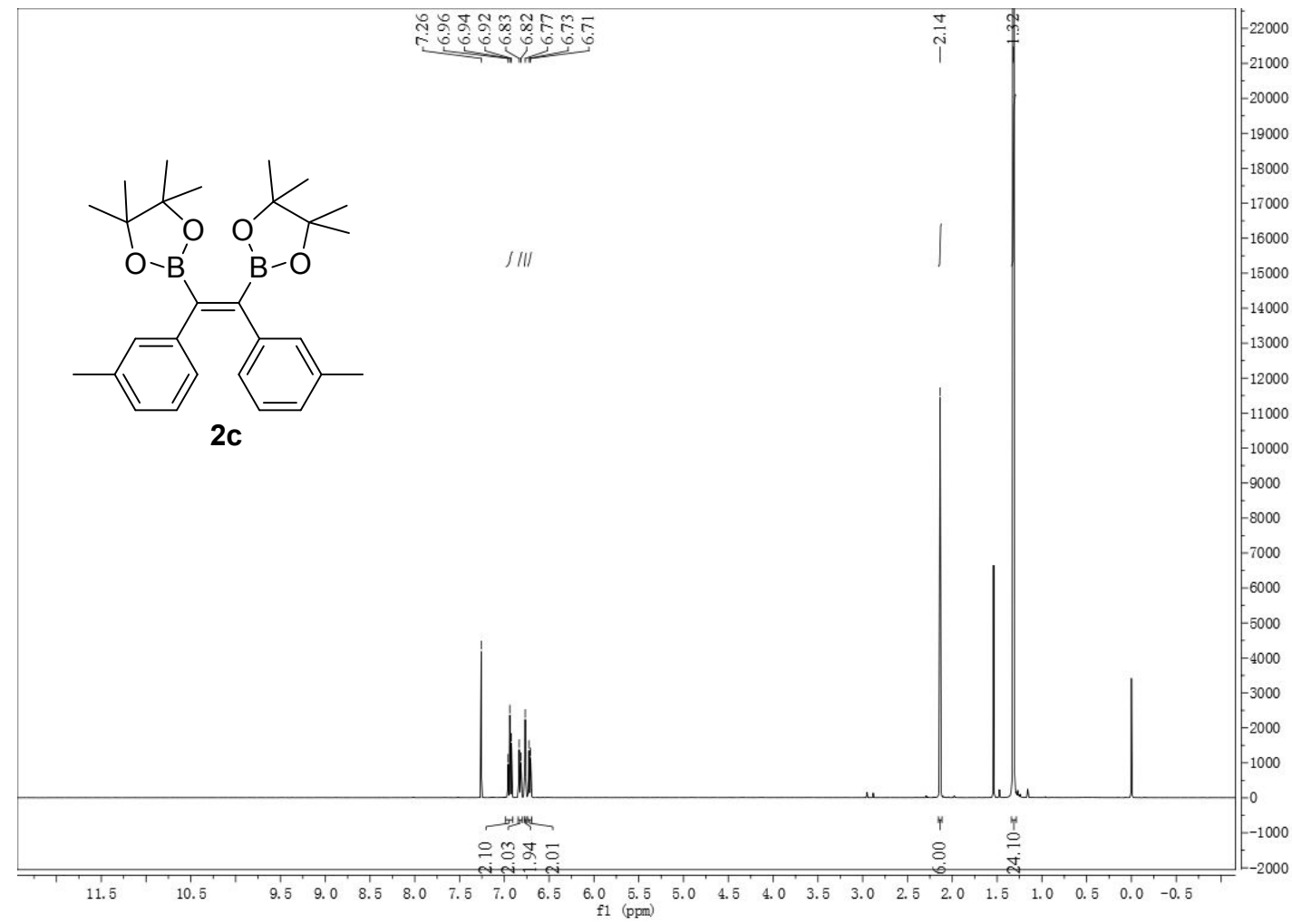

${ }^{13} \mathrm{C}$ - NMR spectrum of $\mathbf{2 c}$

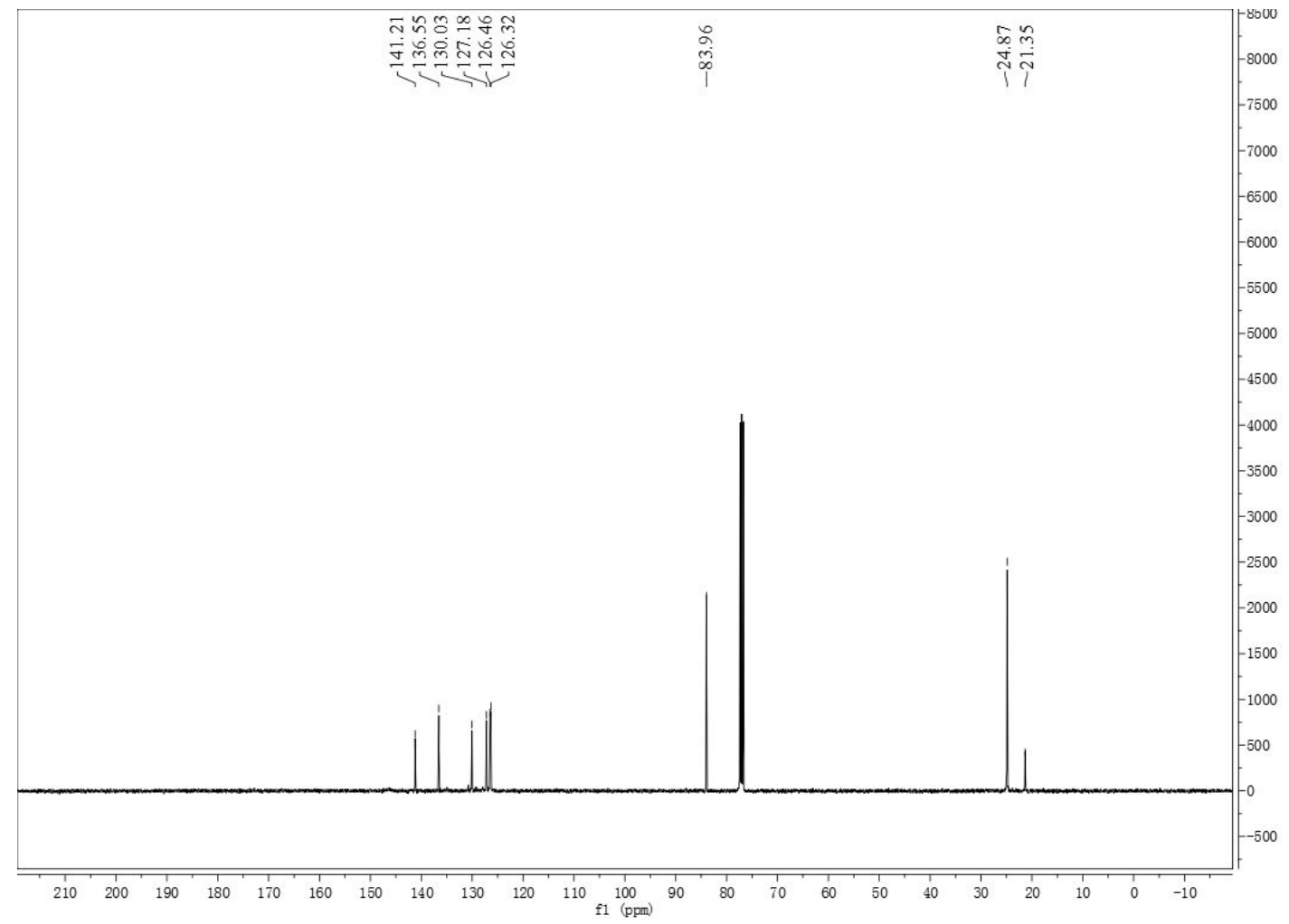


HRMS spectrum of $\mathbf{2 c}$

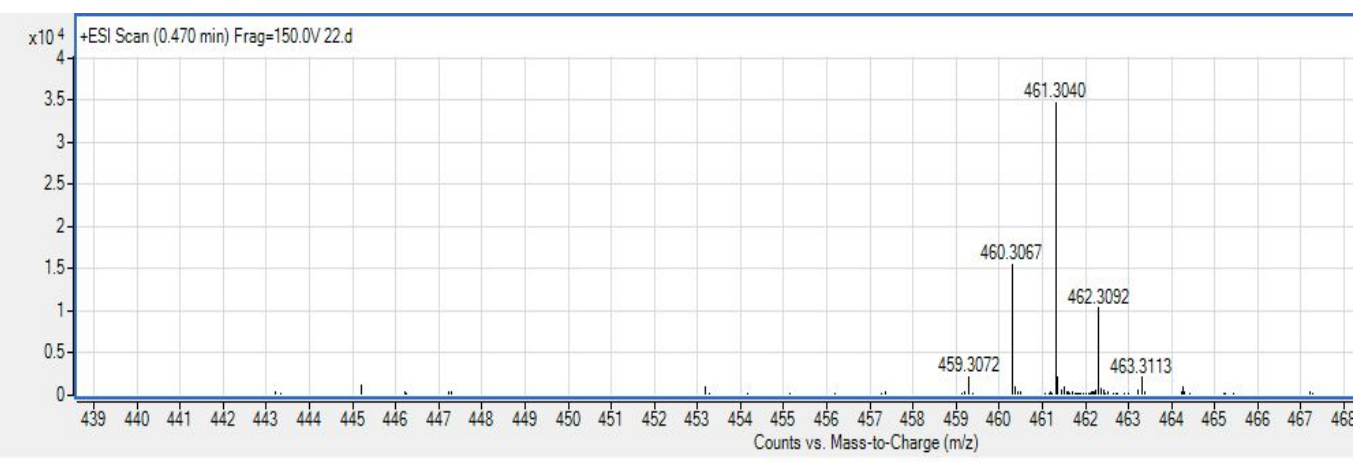

${ }^{1} \mathrm{H}$ - NMR spectrum of $\mathbf{2 e}$

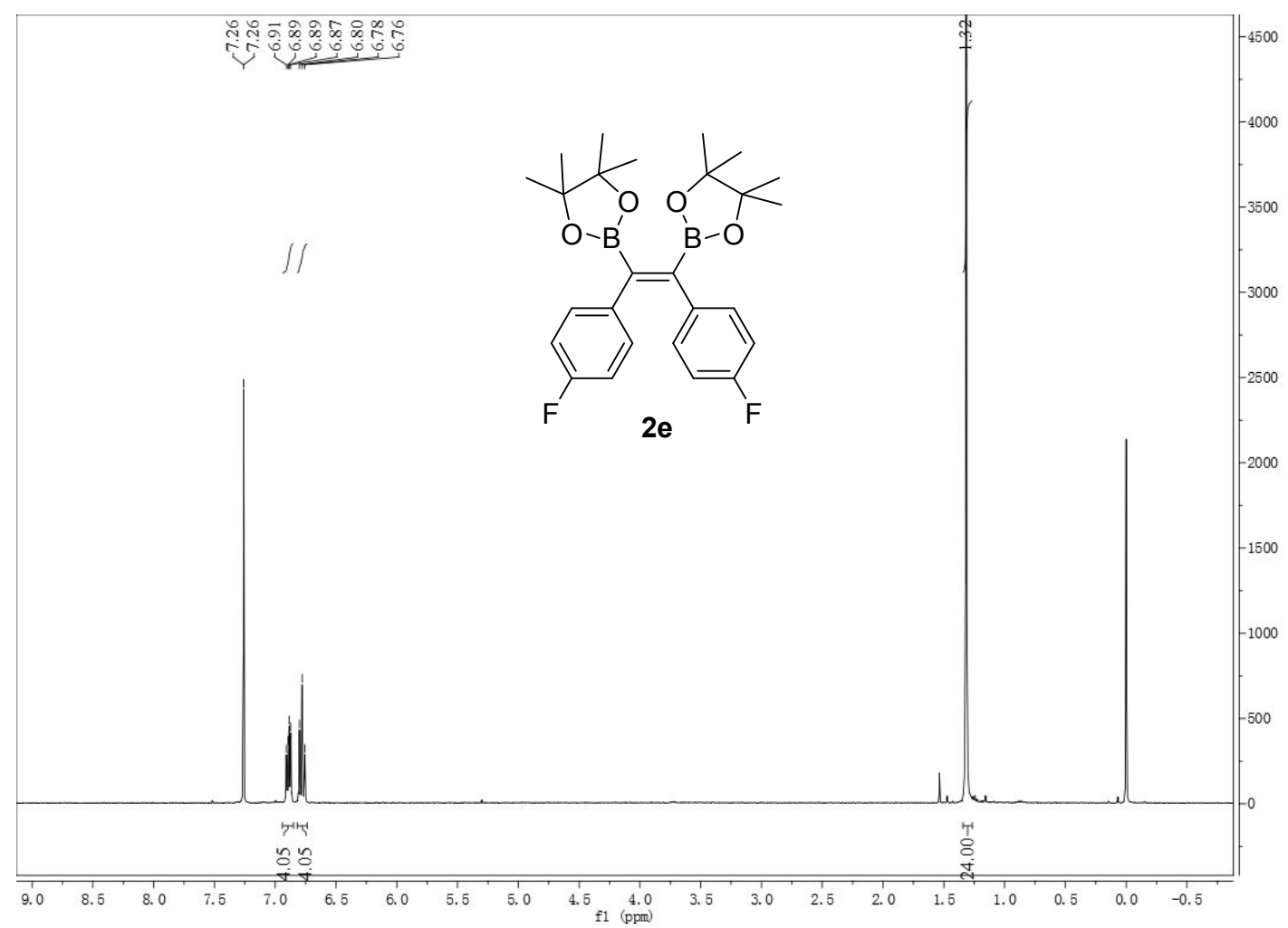


${ }^{13} \mathrm{C}$ - NMR spectrum of $\mathbf{2 e}$

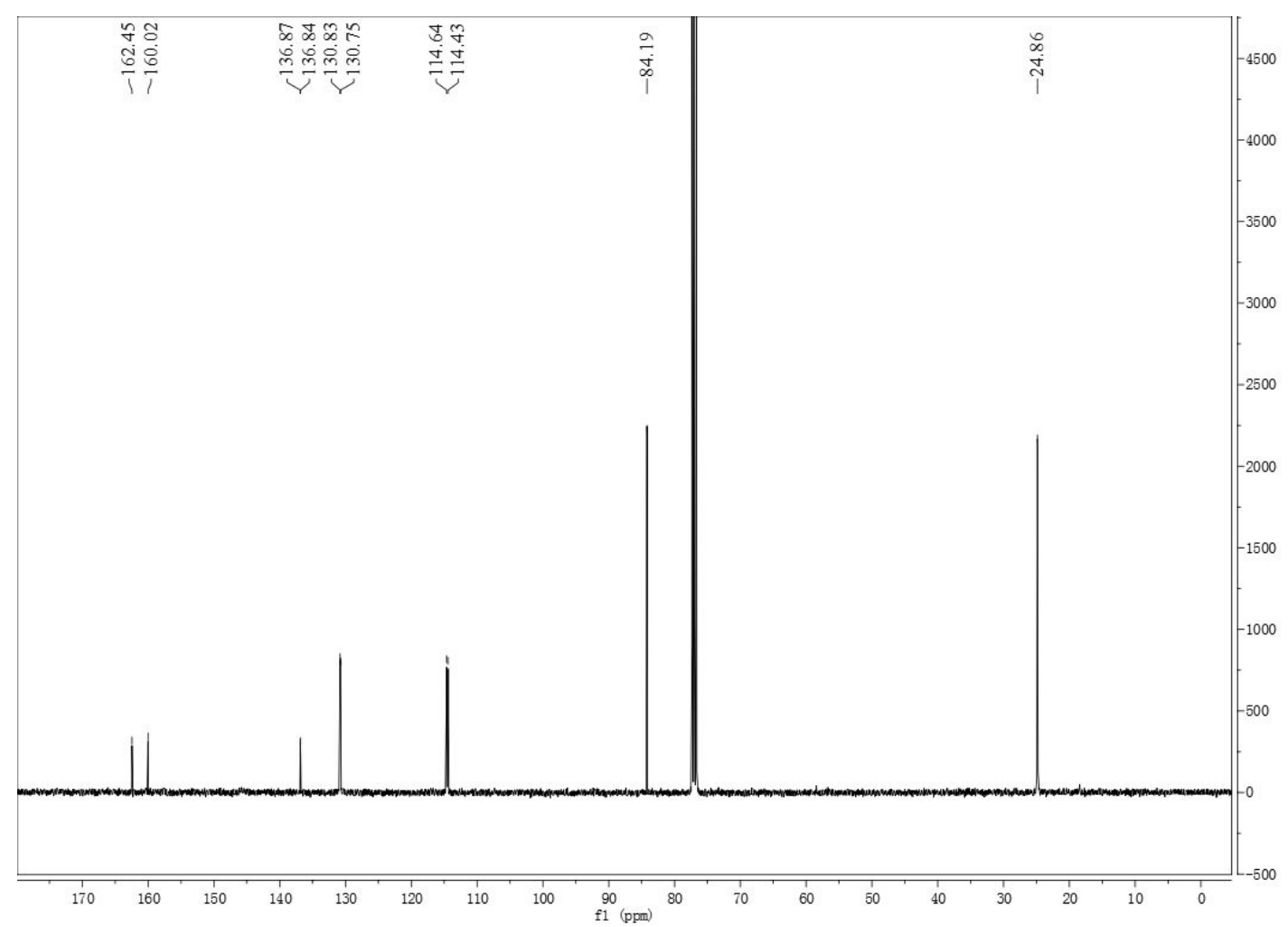

HRMS spectrum of $\mathbf{2 e}$

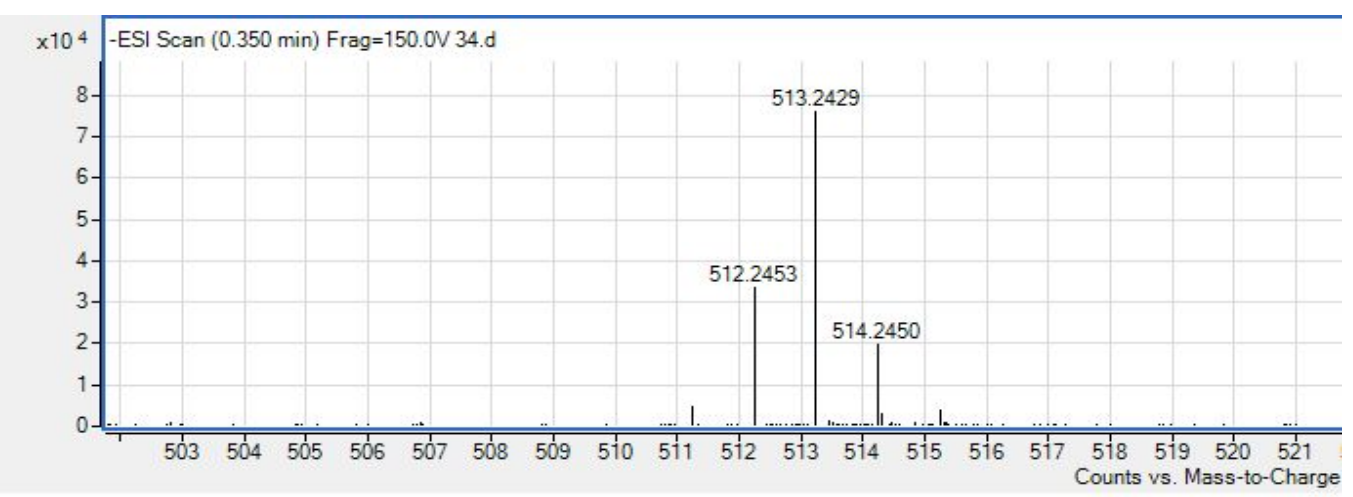


${ }^{1} \mathrm{H}$ - NMR spectrum of $\mathbf{2 f}$

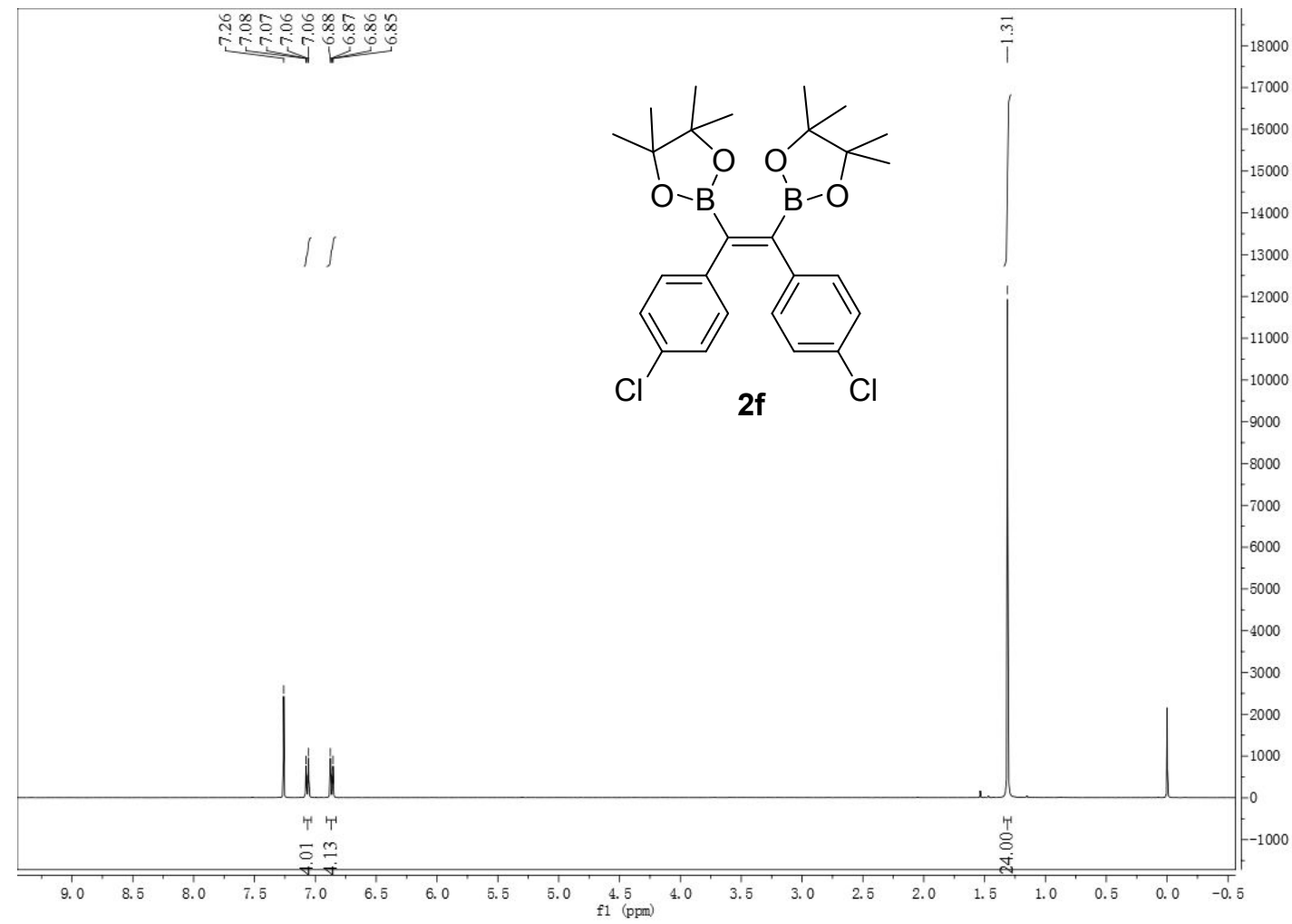

${ }^{13} \mathrm{C}$ - NMR spectrum of $\mathbf{2 f}$

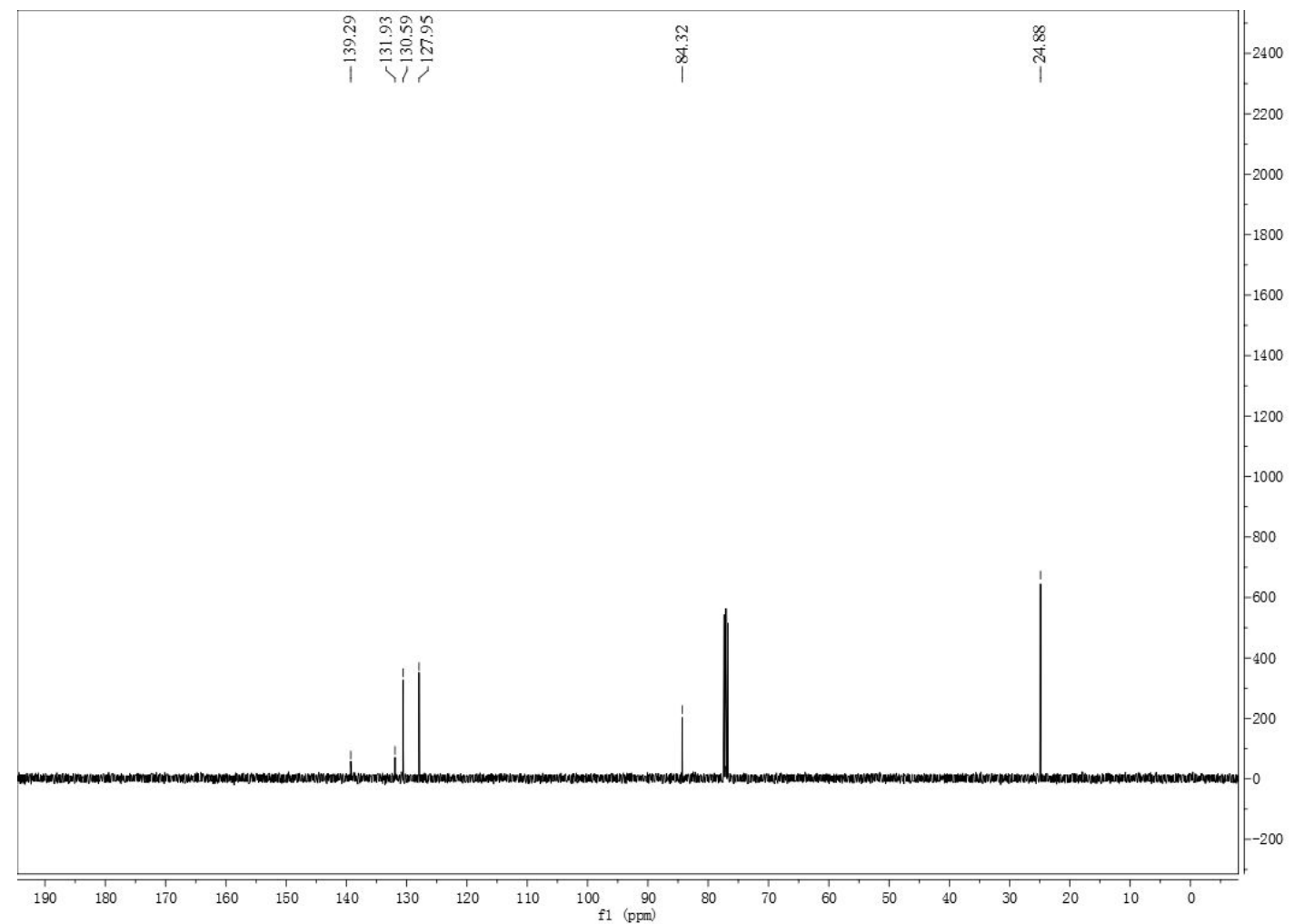


HRMS spectrum of $\mathbf{2 f}$

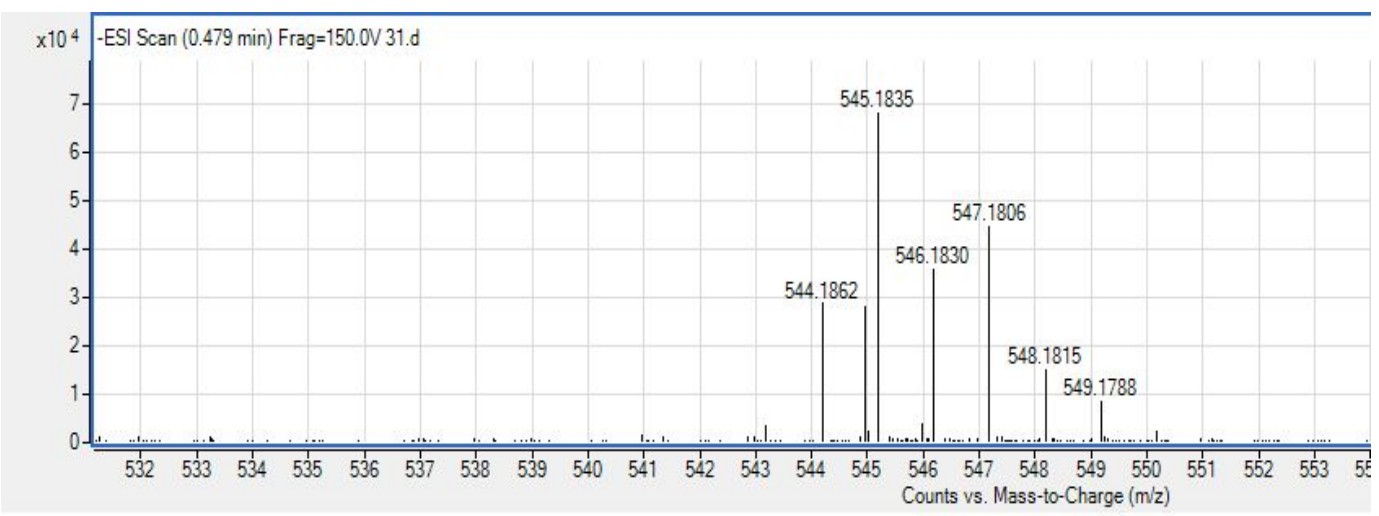

${ }^{1} \mathrm{H}$ - NMR spectrum of $\mathbf{1 8}$

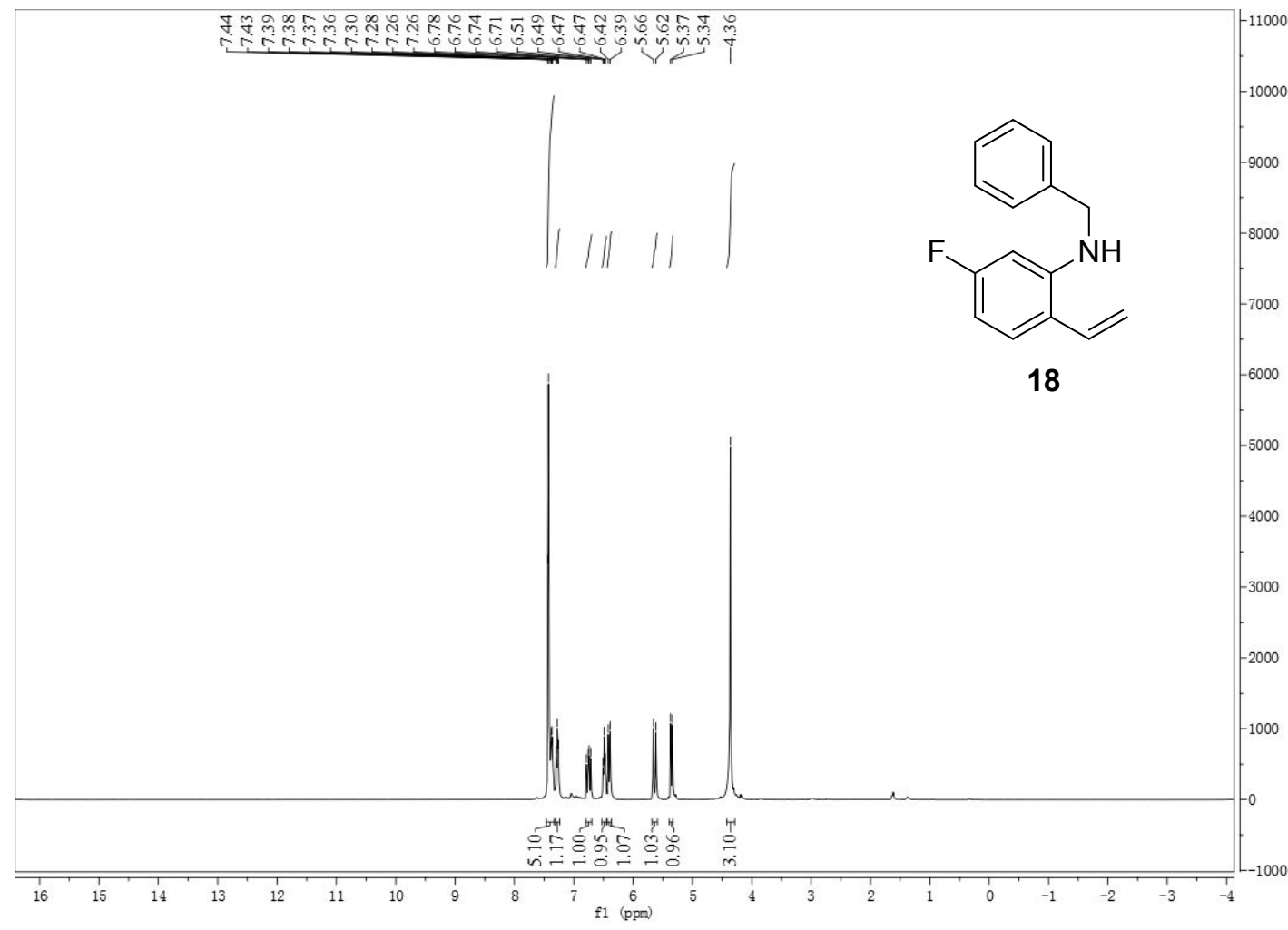


${ }^{13} \mathrm{C}$ - NMR spectrum of $\mathbf{1 8}$

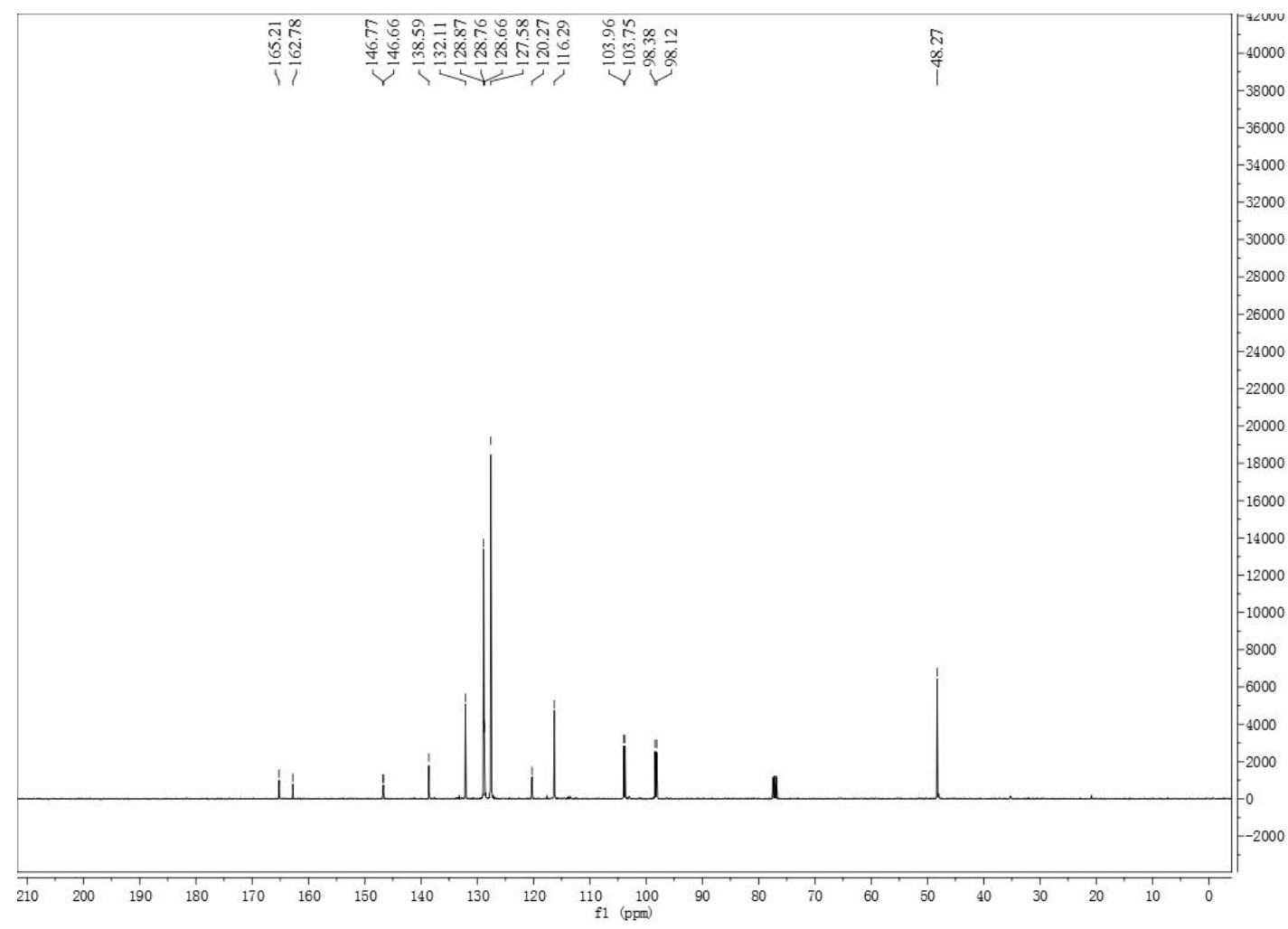

HRMS spectrum of $\mathbf{1 8}$

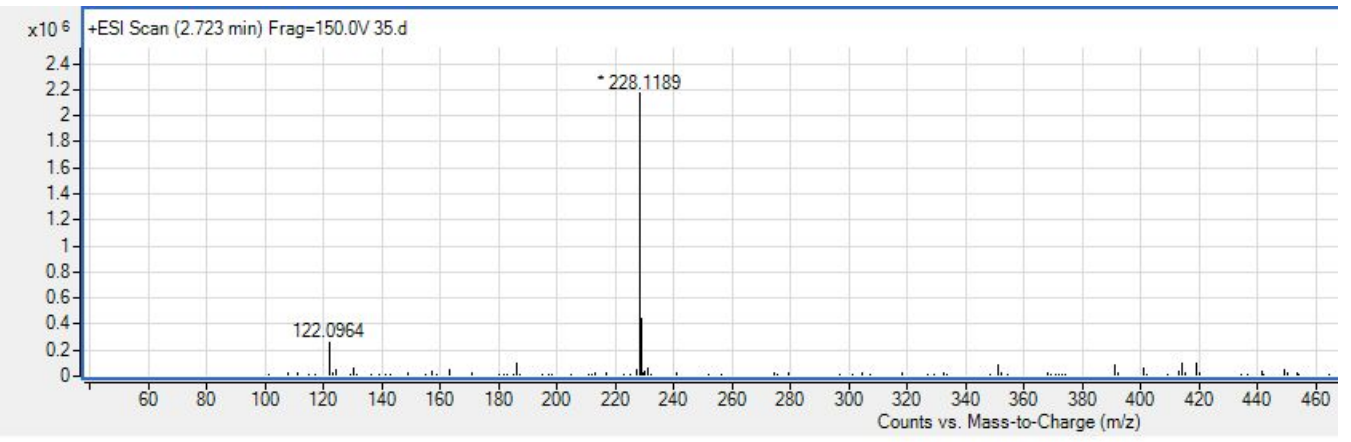


${ }^{1} \mathrm{H}-\mathrm{NMR}$ spectrum of $\mathbf{1 9}$

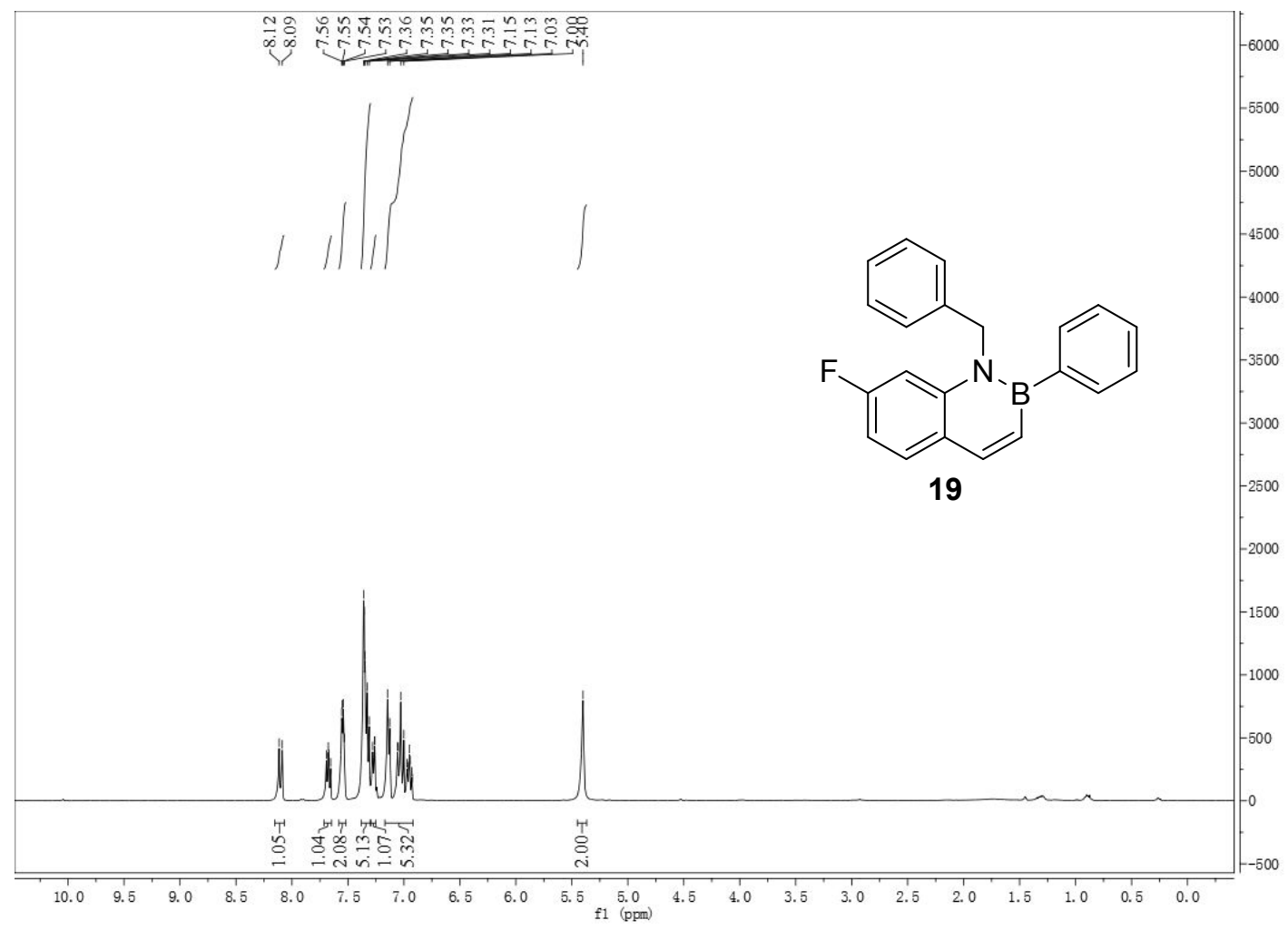

${ }^{13} \mathrm{C}$ - NMR spectrum of $\mathbf{1 9}$

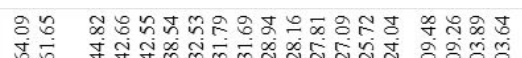

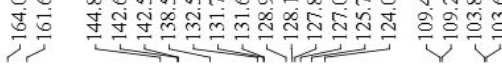

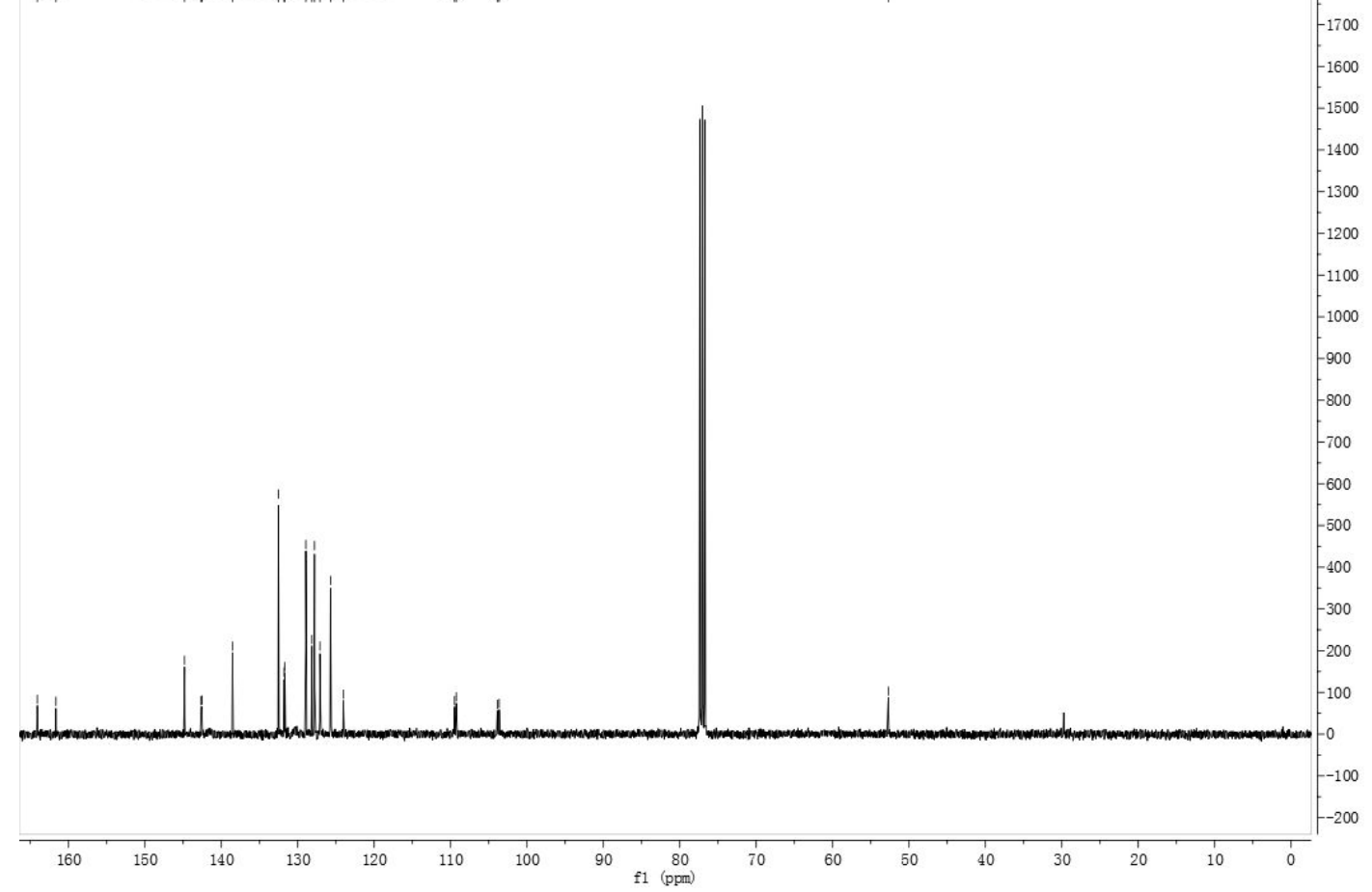


HRMS spectrum of $\mathbf{1 9}$

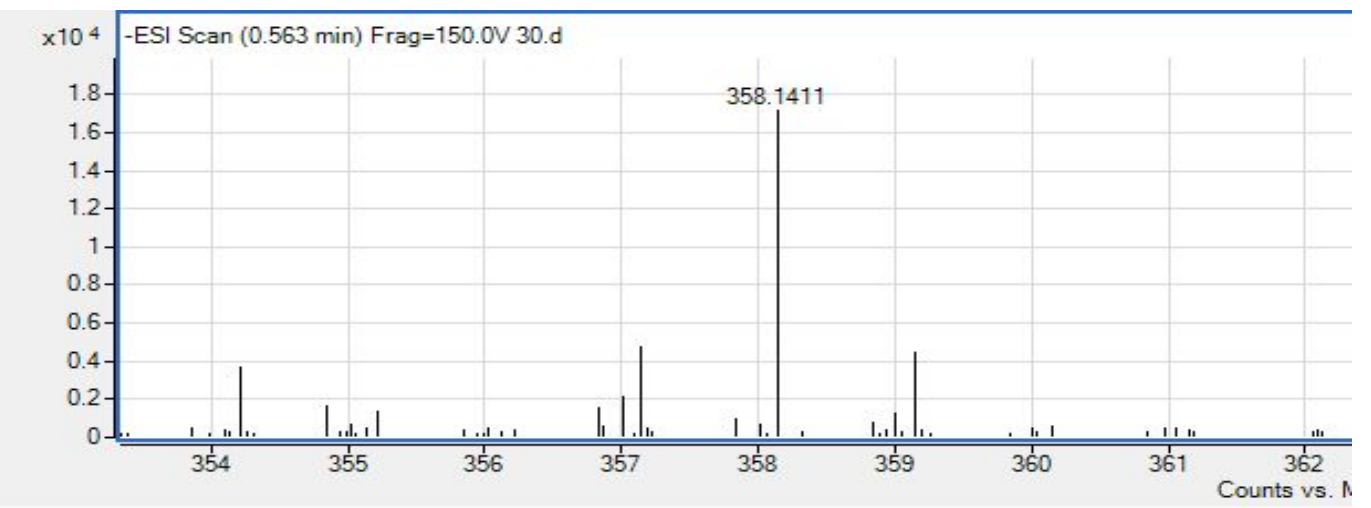

${ }^{1} \mathrm{H}-\mathrm{NMR}$ spectrum of $\mathbf{1 e}$

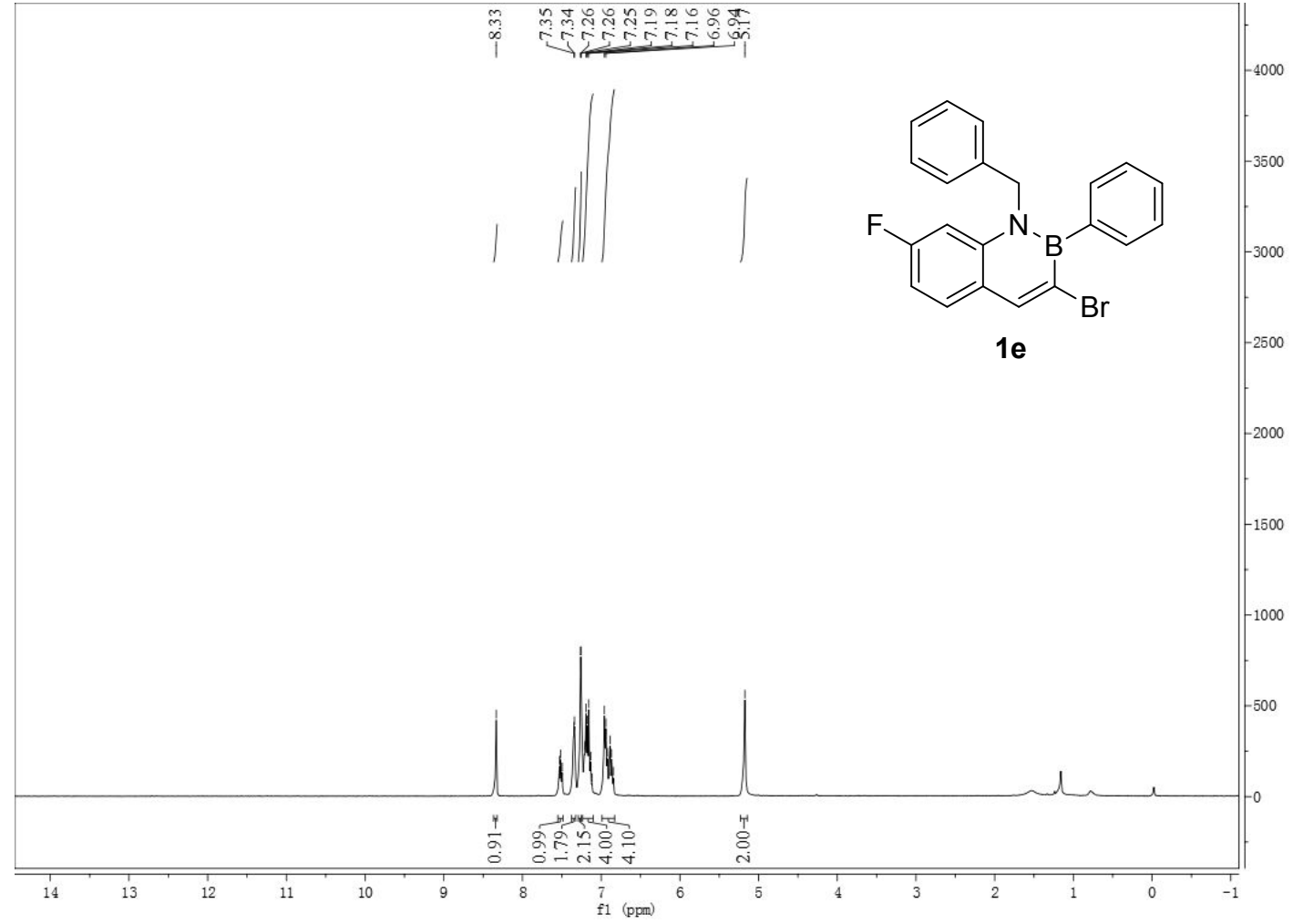


${ }^{13} \mathrm{C}$ - NMR spectrum of $1 \mathrm{e}$

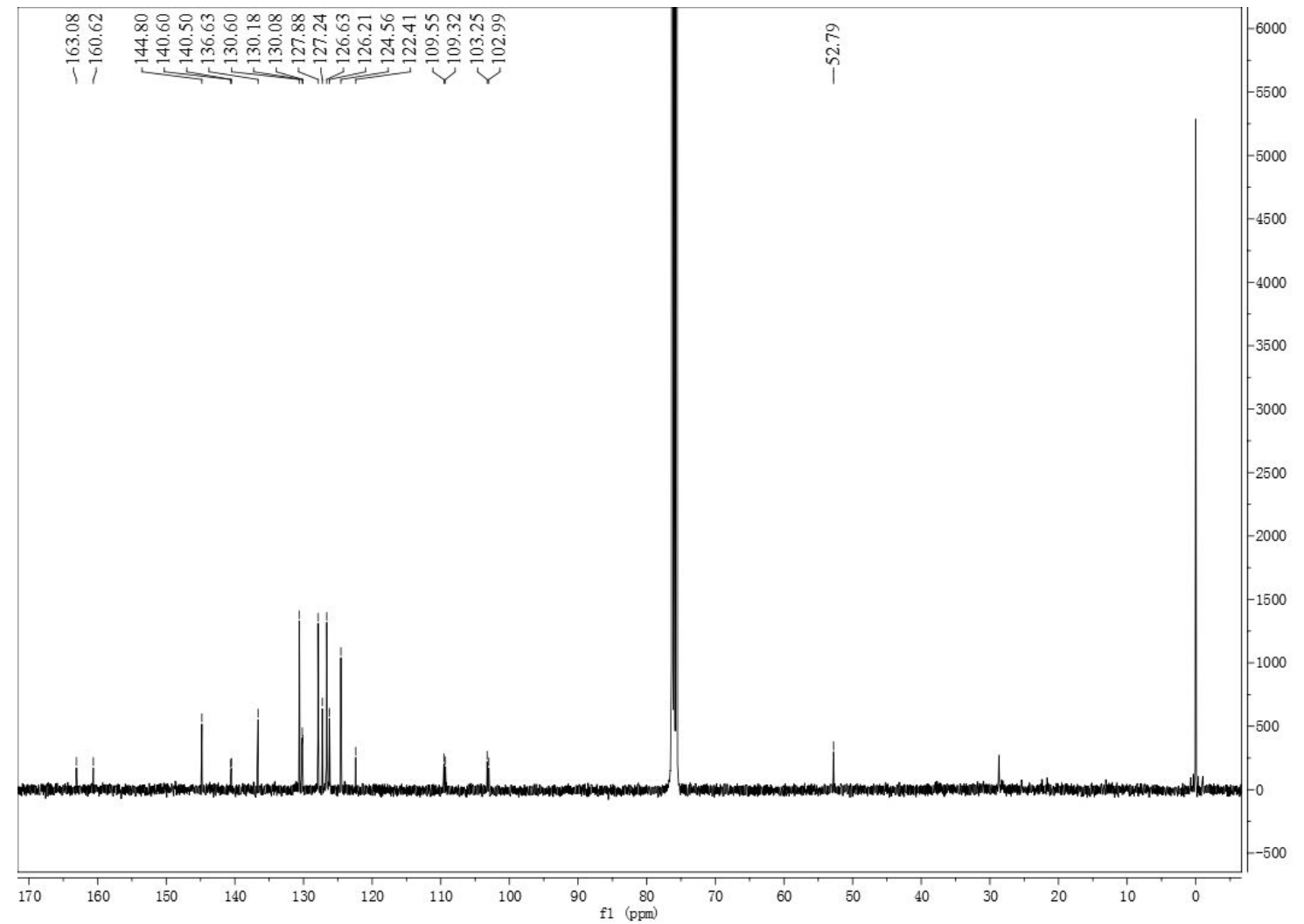

HRMS spectrum of $\mathbf{1 e}$

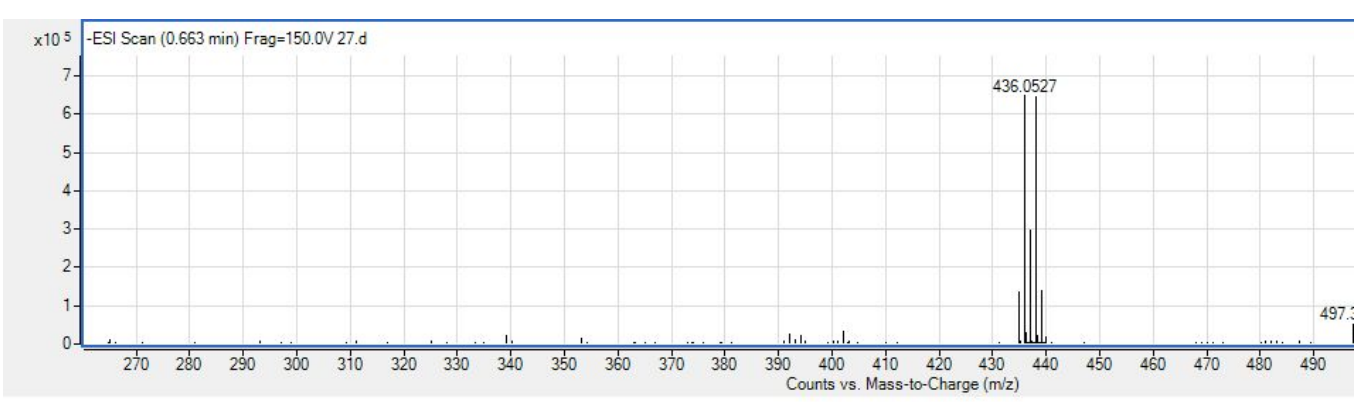


${ }^{1} \mathrm{H}$ - NMR spectrum of $\mathbf{1 f}$

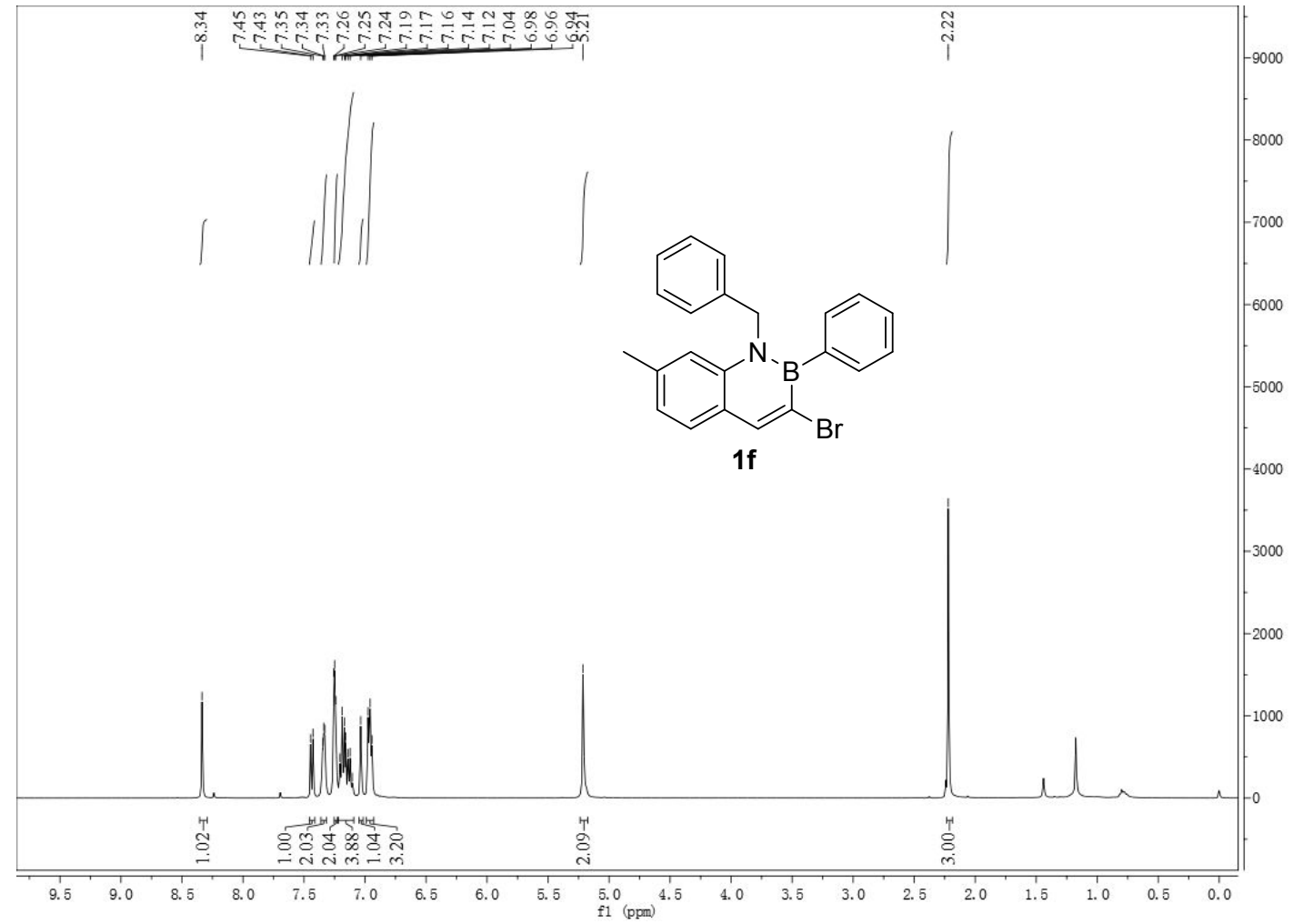

${ }^{13} \mathrm{C}$ - NMR spectrum of $\mathbf{1 f}$

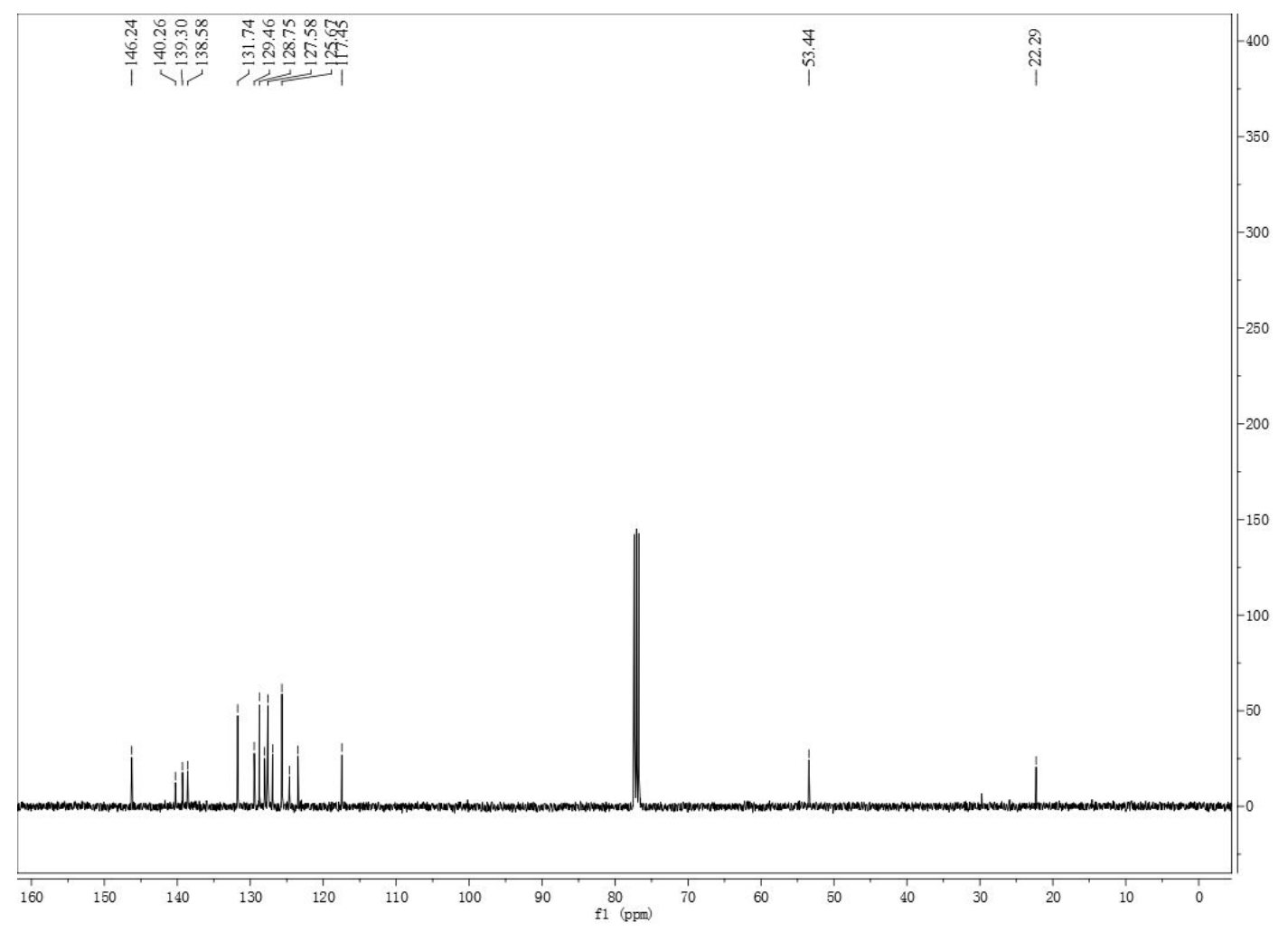


HRMS spectrum of $\mathbf{1 f}$

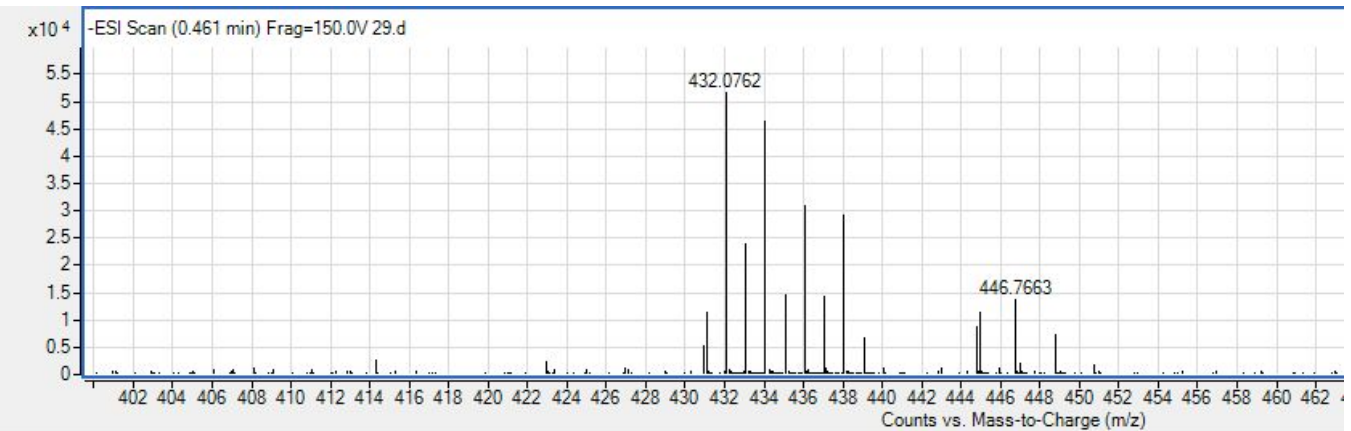

${ }^{1} \mathrm{H}$ - NMR spectrum of 1c

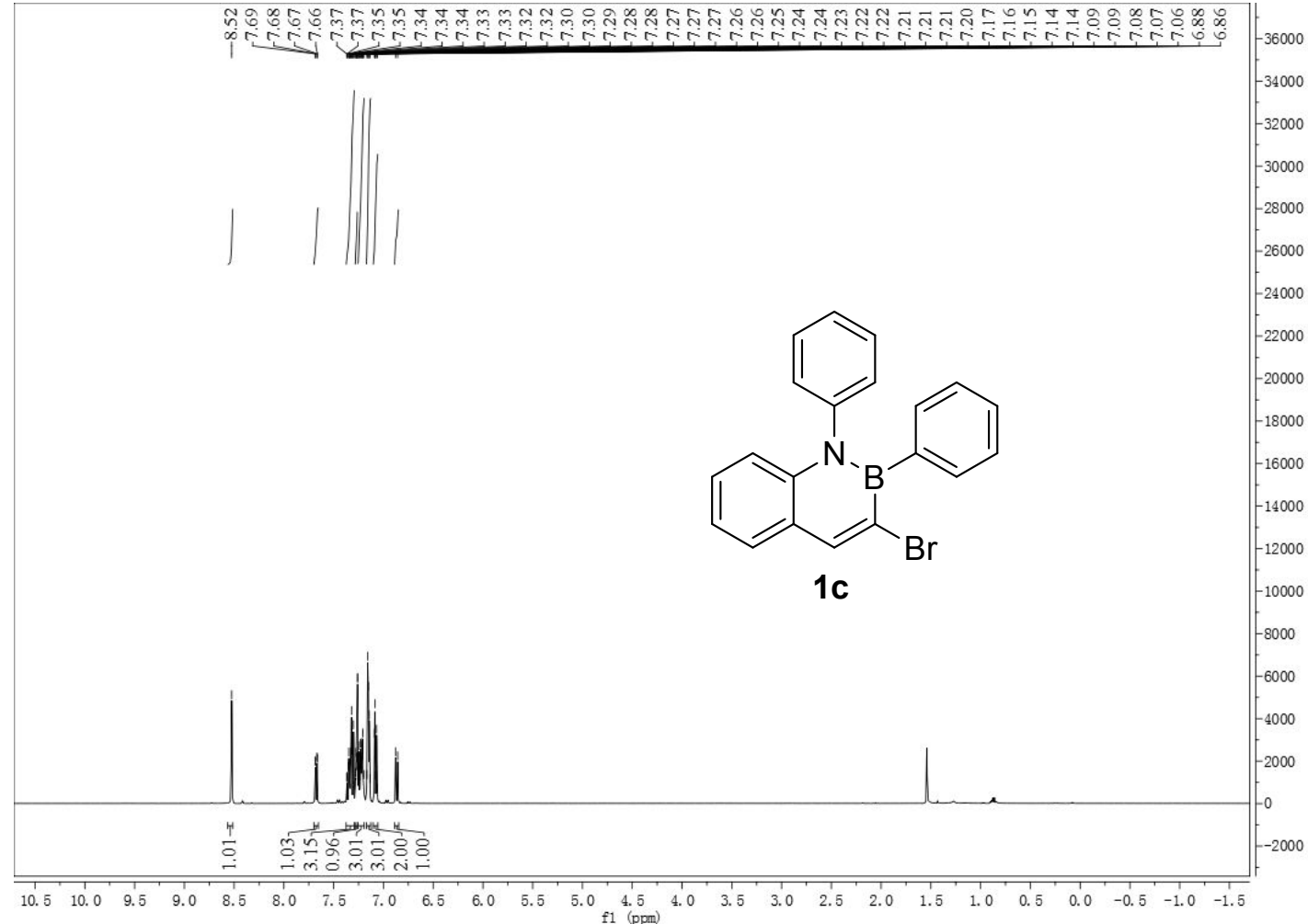


${ }^{13} \mathrm{C}$ - NMR spectrum of $\mathbf{1 c}$

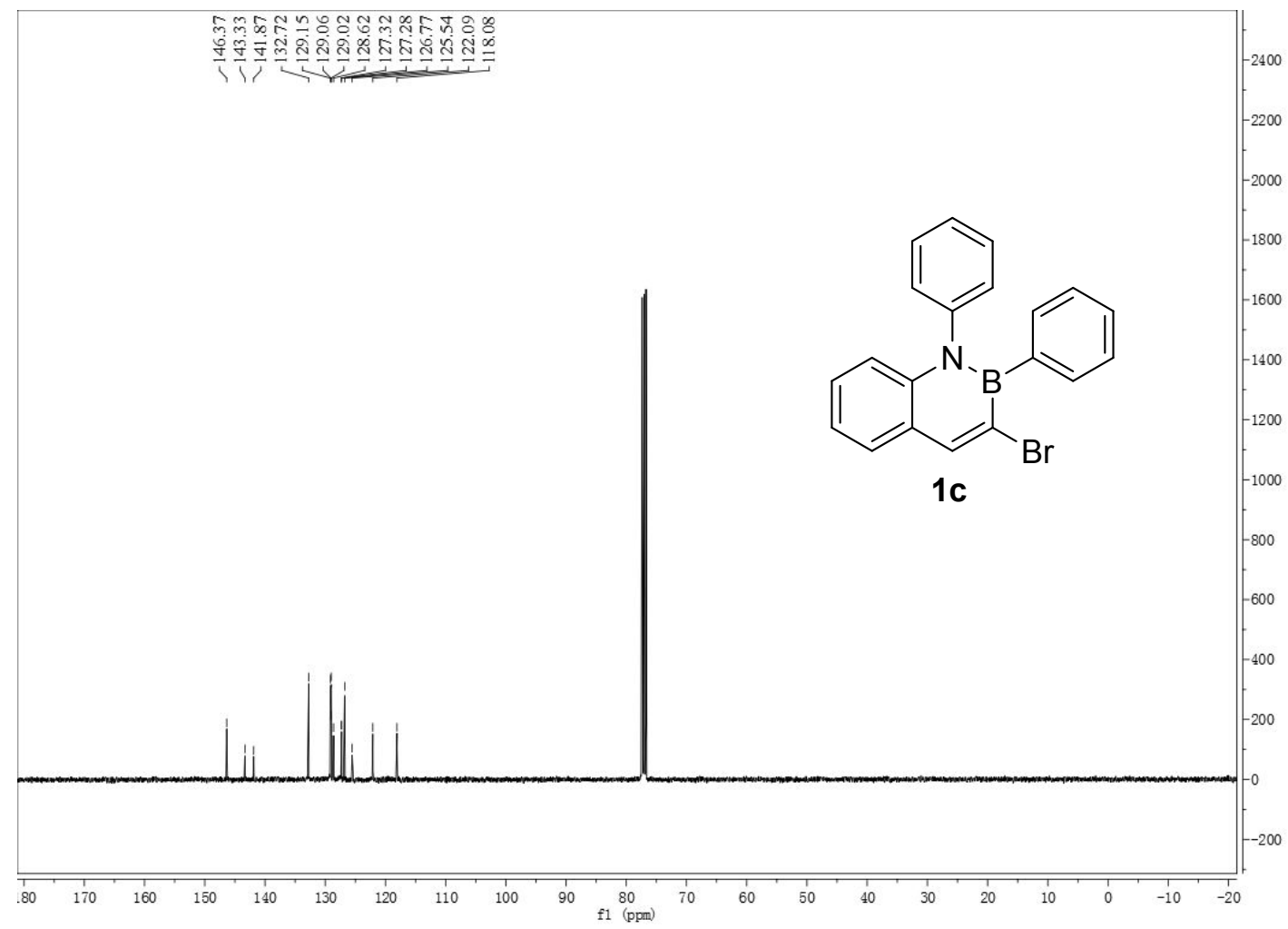

HRMS spectrum of $\mathbf{1 c}$

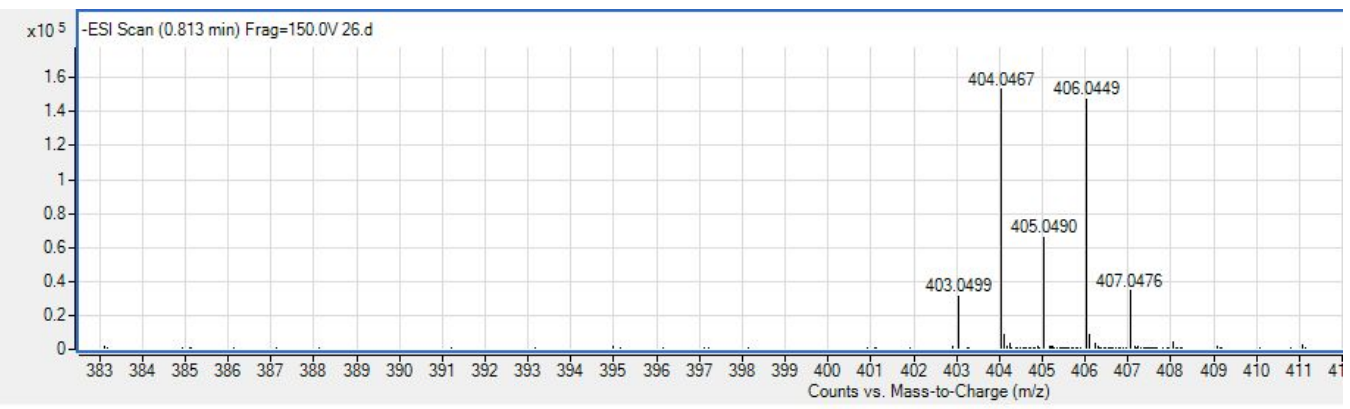

Reference

S1 G. M. Sheldrick. SHELXS-90/96, Program for Structure Solution, Acta. Crystallogr. Sect. A 1990, 46, 467.

S2 M. J. Frisch, G. W. Trucks, H. B. Schlegel, G. E. Scuseria, M. A. Robb, J. R. Cheeseman, J. A. Montgomery, T. Vreven, Jr., K. N. Kudin, J. C. Burant, J. M. Millam,S. S. Iyengar, J. Tomasi, V. Barone, B. Mennucci, M. Cossi, G. Scalmani, N. Rega, G. A. Petersson, H. Nakatsuji, M. Hada, M. Ehara, K. Toyota, R. Fukuda, J. Hasegawa, M. Ishida, T. Nakajima, Y. Honda, O. Kitao, H. Nakai, M. Klene, X. Li, J. E. Knox, H. P. Hratchian, J. B. Cross, C. Adamo, J. Jaramillo, R. Gomperts, R. E. Stratmann, O. Yazyev, A. J. Austin, R. Cammi, C. Pomelli, J. W. Ochterski, P. Y. Ayala, K. Morokuma, G. A. Voth, P. Salvador, J. J. Dannenberg, V. G. Zakrzewski, S. Dapprich, A. D. Daniels, M. C. Strain, O. Farkas, D. K. Malick, A. D. Rabuck, K. Raghavachari, J. B. Foresman, J. V. Ortiz, Q. Cui, A. G. Baboul, S. Clifford, J. Cioslowski, B. B. Stefanov, G. Liu, A. Liashenko, P. Piskorz, I. Komaromi, R. 
L. Martin, D. J. Fox, T. Keith, M. A. Al-Laham, C. Y. Peng, A. Nanayakkara, M. Challacombe, P. M. W.Gill, B. Johnson, W. Chen, M. W. Wong, C. Gonzalez, J. A. Pople, Gaussian 03, Revision C.02, Gaussian, Inc., Wallingford CT, 2004. 